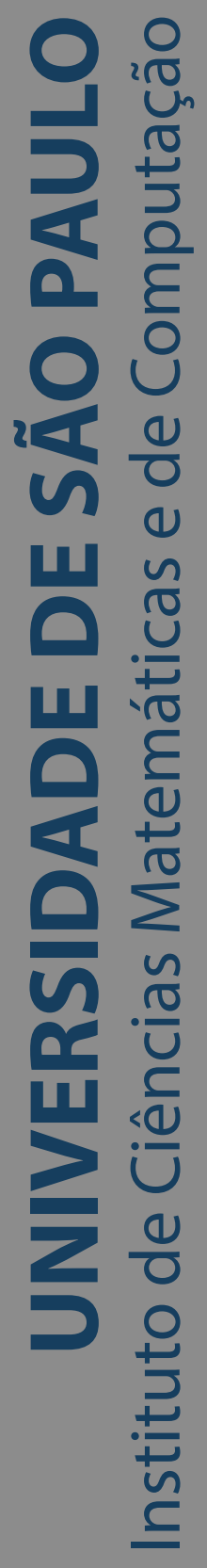

\title{
Ultrafiltros aplicados à Teoria Ergódica de Ramsey
}

\section{José Carlos Fontanesi Kling}

Dissertação de Mestrado do Programa de Pós-Graduação em Matemática (PPG-Mat) 

Assinatura:

\section{José Carlos Fontanesi Kling}

\section{Ultrafiltros aplicados à Teoria Ergódica de Ramsey}

Dissertação apresentada ao Instituto de Ciências Matemáticas e de Computação - ICMC-USP, como parte dos requisitos para obtenção do título de Mestre em Ciências - Matemática. VERSÃO REVISADA

Área de Concentração: Matemática

Orientador: Prof. Dr. Leandro Fiorini Aurichi 
Ficha catalográfica elaborada pela Biblioteca Prof. Achille Bassi e Seção Técnica de Informática, ICMC/USP, com os dados inseridos pelo(a) autor(a)

Kling, José Carlos Fontanesi
K65u Ultrafiltros aplicados à Teoria Ergódica de Ramsey / José Carlos Fontanesi Kling; orientador Leandro Fiorini Aurichi. -- São Carlos, 2020.

$117 \mathrm{p}$.

Dissertação (Mestrado - Programa de Pós-Graduação em Matemática) -- Instituto de Ciências Matemáticas e de Computação, Universidade de São Paulo, 2020.

1. Ultrafiltros. 2. Teoria de Ramsey. 3. Sistemas Dinâmicos. 4. Combinatória. I. Aurichi, Leandro Fiorini, orient. II. Título.

Bibliotecários responsáveis pela estrutura de catalogação da publicação de acordo com a AACR2: Gláucia Maria Saia Cristianini - CRB - 8/4938

Juliana de Souza Moraes - CRB - 8/6176 
José Carlos Fontanesi Kling

\section{Ultrafilters applied to Ergodic Ramsey Theory}

Master dissertation submitted to the Institute of Mathematics and Computer Sciences - ICMC-USP, in partial fulfillment of the requirements for the degree of the Master Program in Mathematics. FINAL VERSION

Concentration Area: Mathematics

Advisor: Prof. Dr. Leandro Fiorini Aurichi

USP - São Carlos

August 2020 

Aos meus pais, que aguardaram este trabalho talvez ainda mais ansiosamente do que eu mesmo. 

Agradeço ao meu orientador, Prof. Dr. Leandro Fiorini Aurichi, pelo apoio e paciência desde a graduação e pela confiança no mestrado.

Agradeço aos meus pais, Benedita Fontanesi e José Carlos Kling pelo amor, apoio e incentivo incondicionais.

À minha companheira de todas as horas, Natália Ayo Schmiedecke, obrigado por tudo, não somente ao que toca esta dissertação.

Por me salvar algumas vezes com burocracias e pela companhia agradeço a Rafael Gironi Dias. Agradeço também a Pedro Henrique Carvalho por me lembrar de prazos e compromissos que eu com certeza teria esquecido, e pelas reclamações mútuas.

E@s amig@s que me ajudaram direta ou indiretamente, deixo também meu agradecimento.

Por fim, o presente trabalho foi realizado com apoio da Coordenação de Aperfeiçoamento de Pessoal de Nível Superior - Brasil (CAPES) - Código de Financiamento 001, e também da Fundação de Amparo à Pesquisa do Estado de São Paulo (FAPESP), processo nº 2018/08886-1. 

"Por que foi que cegámos, Não sei, talvez um dia se chegue a conhecer a razão, Queres que te diga o que penso, Diz,

Penso que não cegámos, penso que estamos cegos, Cegos que vêem, Cegos que, vendo, não vêem." (José Saramago) 



\section{RESUMO}

KLING, J. C. F. Ultrafiltros aplicados à Teoria Ergódica de Ramsey. 2020. 117 p. Dissertação (Mestrado em Ciências - Matemática) - Instituto de Ciências Matemáticas e de Computação, Universidade de São Paulo, São Carlos - SP, 2020.

Os ultrafiltros têm aplicações em diversas áreas da matemática, como Topologia, Teoria de Modelos ou Análise Non-standard. Neste trabalho veremos aplicações a Dinâmica Topológica e Teoria de Ramsey para então chegarmos aos resultados clássicos de Teoria Ergódica de Ramsey. Apresentamos uma introdução auto contida à área, baseada em grande parte nos trabalhos de Neil Hindman, Dona Strauss e Vitaly Bergelson.

Palavras-chave: Ultrafiltros, Teoria de Ramsey, Sistemas Dinâmicos, Combinatória. 



\section{ABSTRACT}

KLING, J. C. F. Ultrafilters applied to Ergodic Ramsey Theory. 2020. 117 p. Dissertação (Mestrado em Ciências - Matemática) - Instituto de Ciências Matemáticas e de Computação, Universidade de São Paulo, São Carlos - SP, 2020.

Ultrafilters have applications in several fields of mathematics, such as Topology, Model Theory or Non-Standard Analysis. In this work it will be presented applications to Topological Dynamics and Ramsey Theory, aiming for the classical results from Ergodic Ramsey Theory.

We present a self-contained introduction to the area, based mainly on the works of Neil Hindman, Dona Strauss and Vitaly Bergelson.

Keywords: Ultrafilters, Ramsey Theory, Dynamical Systems, Combinatorics. 

$\hat{x}$ - Ultrafiltro principal

$\beta X$ - Compactificação de StoneČech de um espaço topológico completamente regular $X$

$\widehat{A}$ - Aberto básico da topologia de $\beta X$

$(S,+)$ - Semigrupo composto pelo conjunto $S$ e operação associativa +

$\lambda_{s}$ - Função em um semigrupo determinada por $\lambda_{s}(r)=s+r$

$\rho_{s}$ - Função em um semigrupo determinada por $\rho_{s}(r)=r+s$

$E(S)$ - Conjunto dos elementos idempotentes de um semigrupo $S$.

$K(S)$ - O menor ideal de um semigrupo $S$.

$\Lambda(S)$ - Centro topológico do semigrupo $S$

$\left(X,\left\langle T_{s}\right\rangle_{s \in S}\right)$ - Sistema dinâmico sobre um semigrupo $S$

$[X]^{k}$ - Conjunto de todos os subconjuntos de $X$ com cardinalidade $k$

$F S(H)$ - Conjunto das somas de finitos elementos distintos de um conjunto $H$ em um semigrupo

$I P+-$ Conjunto dos elementos idempotentes de $(\beta \mathbb{N},+)$

$I P \cdot$ - Conjunto dos elementos idempotentes de $(\beta \mathbb{N}, \cdot)$

$I P m+-$ Conjunto dos elementos idempotentes minimais de $(\beta \mathbb{N},+)$

IPm. - Conjunto dos elementos idempotentes minimais de $(\beta \mathbb{N}, \cdot)$

$d(A)$ — Densidade natural em $\mathbb{N}$

$\bar{d}(A)$ - Densidade superior em $\mathbb{N}$

$\Delta$ — Conjunto de subconjuntos de $\mathbb{N}$ com densidade superior não nula $d^{*}(A)$ - Densidade de Banach superior em $\mathbb{N}$

$\Delta^{*}$ — Conjunto de subconjuntos de $\mathbb{N}$ com densidade de Banach superior não nula $(X, \mathscr{B}, \mu, T)$ - Sistema dinâmico em que $(X, \mathscr{B}, \mu)$ é um espaço de medida e $T: X \rightarrow X$ preserva medida

$\mathscr{D}(N)$ - Conjunto das diferenças de elementos de $N$ 

INTRODUÇÃO . . . . . . . . . . . . . . . . . 19

CONSTRUÇÕES BÁSICAS . . . . . . . . . . . . . . . 23

Ultrafiltros . . . . . . . . . . . . . . . . . . . . 23

$2.2 \quad$ Compactificação de Stone-Čech $\beta X \ldots \ldots \ldots$

2.3 Compactificação de Semigrupos . . . . . . . . . . . . . . . 34

3 ESTRUTURA AlgÉBRICA DE SEMIGRUPOS . . . . . . . . 37

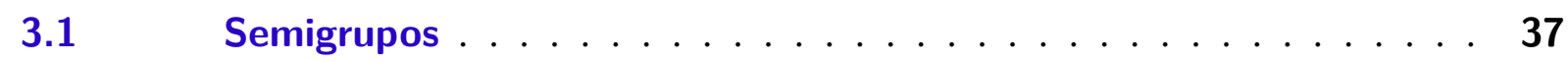

3.2 Semigrupos topológicos à direita . . . . . . . . . . . . . 43

Alguns problemas em aberto . . . . . . . . . . . . 49

4 SISTEMAS DINÂMICOS . . . . . . . . . . . . . . . 51

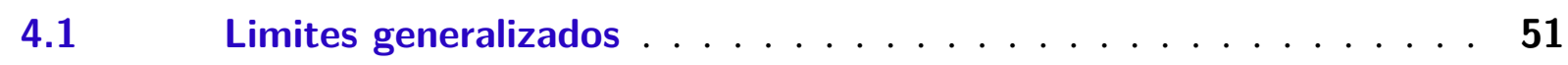

$4.2 \quad$ Universalidade de $\beta S \ldots \ldots \ldots \ldots \ldots$

$4.3 \quad$ Sistemas Dinâmicos Minimais . . . . . . . . . . . . . . 61

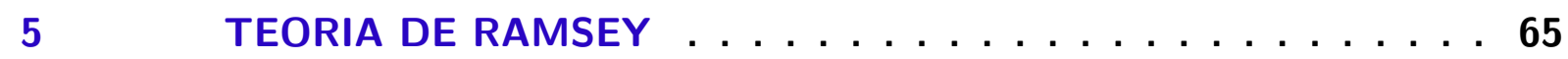

$5.1 \quad$ Teorema de Ramsey .................. 66

5.2 Teorema de Van Der Waerden . . . . . . . . . . . . . . 68

5.3 Teorema de Hindman . . . . . . . . . . . . . . 72

5.4 Teorema de Hindman para soma e produto . . . . . . . . . . . 74

5.5 Generalização dos Teoremas de Hindman e Van der Waerden . . 78

5.6 Teorema de Hales-Jewett . . . . . . . . . . . . . 79

5.7 Recorrência e Teoria de Ramsey . . . . . . . . . . . . . 86

$5.8 \quad$ Teorema de Gallai . . . . . . . . . . . . . . . . . 89

$5.9 \quad$ Alguns problemas em aberto .............. 93

6 TEOREMAS DE DENSIDADE . . . . . . . . . . . . . 97

6.1 Densidade de Banach . . . . . . . . . . . . . . 97

6.2 Princípio da Correspondência de Furstenberg e Teorema de Szemerédi100

6.3 Teorema de Schur . . . . . . . . . . . . . . . . 103

6.4 Teorema de Brauer ................... 106

6.5 Teorema de Hindman e densidade . . . . . . . . . . . 107 
REFERÊNCIAS . . . . . . . . . . . . . . . . . . 115 


\section{1}

\section{INTRODUÇÃO}

O presente trabalho é um estudo sobre ultrafiltros e suas aplicações, com foco especial para a Teoria de Ramsey e sistemas dinâmicos: áreas que desde os anos 70 se mostram intimamente relacionadas.

Um dos aspectos que tornam este um ótimo tema de estudo é que precisamos mobilizar conhecimentos de diversas áreas distintas, como Topologia, Teoria dos Conjuntos, Sistemas Dinâmicos e Combinatória, o que possibilita o contato com pesquisas muito diversas, e permite que uma gama variada de alunos e pesquisadores tenham como tirar proveito de seu estudo.

A exposição feita neste trabalho segue bastante de perto o trabalho Hindman e Strauss (2012), a principal referência na área, contendo desde as definições mais básicas - como de ultrafiltro, Compactificação de Stone-Čech e semigrupos - até resultados originais. Outros trabalhos importantes são as publicações de Vitaly Bergelson e o livro Furstenberg (1981), que focam especialmente aspectos da Teoria Ergódica.

O único pré-requisito exigido neste trabalho é um bom conhecimento de topologia. Praticamente todos os espaços citados neste trabalho são discretos, ou compactos de Hausdorff, portanto é necessária uma boa familiaridade com essas definições. Utilizaremos também diversas propriedades de funções contínuas, o Teorema de Tychonoff e o conceito de base de uma topologia. As notas de aula Aurichi (2020), elaboradas pelo Professor Leandro Aurichi, são bastante concisas e cobrem todo o necessário para nossos objetivos, além de utilizar uma notação consistente com a utilizada aqui.

A Teoria de Ramsey tem origem em diversos resultados independentes datando do início do século XX, com motivações advindas de áreas completamente distintas, por exemplo, o Teorema de Schur teve sua aplicação a Teoria dos Números, enquanto o Teorema de Ramsey é, originalmente, um lema para um resultado de lógica. Estes resultados foram unificados em uma mesma teoria, pois possuem uma formulação que os torna muito similares e podem ser aplicados a áreas para os quais não foram intencionados, como Teoria de Grafos ou Teoria dos Jogos.

Um tipo de problema estudado pela Teoria de Ramsey, que nos ocuparemos neste trabalho, 
tem por interesse determinar se, ao se "quebrar" uma estrutura em finitos "pedaços", podemos garantir que ao menos um desse "pedaços" necessariamente possui alguma estrutura combinatória interessante. Como exemplo, o Teorema de Van der Waerden afirma que se particionarmos os números naturais em finitos conjuntos, então um deles conterá progressões aritméticas de comprimento arbitrário.

Esses problemas tem um enunciado comum:

Teorema 1.1. Seja $X$ um conjunto e $\mathscr{A} \subset \mathscr{P}(X)$ uma família de subconjuntos de $X$. Para toda partição finita $X=\bigcup_{i=1}^{r} A_{i}$, existe algum $j \in\{1, \ldots, r\}$ e $A \in \mathscr{A}$ de modo que $A \subset A_{j}$.

Para conseguir os diferentes enunciados temos que especificar o conjunto $X$ - como $X=\mathbb{N}$ ou exigindo que $X$ tenha também a estrutura de semigrupo - e a família $\mathscr{A}$ - como no Teorema de Van der Waerden, em que

$\mathscr{A}=\{A \subset \mathbb{N} \mid A$ contém progressões aritméticas de comprimento arbitrário $\}$.

Um ultrafiltro sobre um conjunto $X$ é uma família maximal de subconjuntos de $X$ fechada por interseções e pela inclusão (Definição 2.6). Como mostraremos no próximo capítulo, qualquer ultrafiltro sobre $X$ é uma família que satisfaz o teorema acima, mas ,além disso, qualquer uma dessas famílias é determinada por algum ultrafiltro. Mais precisamente, e deixando claro o porquê do interesse em se aplicar os ultrafiltros neste contexto, temos a seguinte equivalência:

Teorema 1.2. Seja $X$ um conjunto e $\mathscr{A} \subset \mathscr{P}(X)$ uma família de subconjuntos de $X$. A família $\mathscr{A}$ satisfaz o teorema anterior se, e somente se, existe algum ultrafiltro $\mathscr{U}$ sobre $X$ de modo que para todo $U \in \mathscr{U}$, existe $A \in \mathscr{A}$ com $A \subset U$.

O que fizemos aqui foi transformar um problema sobre partições finitas de um conjunto em um problema sobre a existência de ultrafiltros. Talvez o melhor exemplo da utilidade deste resultado seja o Teorema de Hindman.

Para todo conjunto $H \subset \mathbb{N}$, definimos o conjunto

$$
F S(H)=\left\{\sum_{h \in F} h \in \mathbb{N} \mid F \subset H \text { é finito }\right\}
$$

ou seja, o conjunto de todas as somas de finitos elementos dois a dois distintos de $H$. O Teorema de Hindman afirma que para qualquer partição finita $\bigcup_{i=1}^{r} A_{i}$, existe algum $j \in\{1, \ldots, r\}$ de modo que $A_{j}$ contém o conjunto $F S(H)$ para algum $H \subset \mathbb{N}$ infinito.

Este problema permaneceu em aberto por várias décadas, tendo sido estudado por diversos matemáticos, incluindo David Hilbert, tendo sido demonstrado por Neil Hindman em 1974 (HINDMAN, 1974), envolvendo um longo e complicado argumento combinatório.

O conjunto dos ultrafiltros sobre os naturais, que denotaremos por $\beta \mathbb{N}$, admite uma topologia que o torna um espaço topológico compacto de Hausdorff satisfazendo duas propriedades: $\mathbb{N} \subset \beta \mathbb{N}$ e, dada qualquer função $f: \mathbb{N} \rightarrow Y$, em que $Y$ é um compacto de Hausdorff, existe uma única função 
contínua $\tilde{f}: \beta \mathbb{N} \rightarrow Y$ tal que $f \uparrow_{\mathbb{N}}=f$, ou seja, toda função de $\mathbb{N}$ para um espaço compacto de Hausdorff, existe uma única extensão desta função para $\beta \mathbb{N}$ que é contínua.

Essas propriedades nos permitem estender a soma para $\beta \mathbb{N}$, o que torna $\beta \mathbb{N}$ um semigrupo. Essa construção nos permite utilizar as teorias sobre semigrupos topológicos para garantir a existência de ultrafiltros.

Voltando ao Teorema de Hindman, como foi contado em Hindman (2005), desde 1971 Fred Galvin conjecturava a existência de um ultrafiltro com a seguinte propriedade: se um conjunto $A$ pertence ao ultrafiltro $\mathscr{U}$, então $\{x \in \mathbb{N} \mid-x+A \in \mathscr{U}\}$ pertence a $\mathscr{U}$. Caso a existência fosse demonstrada, o Teorema de Hindman seguiria como consequência.

Apenas em 1975, ao conversar com Steven Glazer, Galvin conseguiu a resposta. Como mostraremos na Seção 5.3, qualquer ultrafiltro idempotente, ou seja, um ultrafiltro tal que $\mathscr{U}+\mathscr{U}=\mathscr{U}$, satisfaz a propriedade acima, mas o que Glazer sabia era que qualquer semigrupo topológico à direita compacto, como é o caso de $\beta \mathbb{N}$, possui elementos idempotentes.

A demonstração de Galvin e Glazer tem duas grandes vantagens em comparação com a original: ela é bastante mais simples e é válida não somente para $\mathbb{N}$, mas para qualquer semigrupo. Também foram desenvolvidas demonstrações utilizando as propriedades algébricas de $\beta \mathbb{N}$ para diversos outros resultados.

Um princípio da Teoria de Ramsey é que certas propriedades combinatórias são inevitáveis em conjuntos "grandes". No caso dos enunciados anteriores, intuitivamente temos a ideais de que se "quebrarmos" uma estrutura infinita em finitas partes, uma dessas partes deverá ser "grande", e, na verdade, é esse o motivo porque surgem essas propriedades.

Nos números naturais, a noção de tamanho mais natural é a densidade $\bar{d}: \mathscr{P}(\mathbb{N}) \rightarrow[0,1]$, definida como, para todo $A \subset \mathbb{N}$,

$$
\bar{d}(A)=\lim _{n \rightarrow \infty} \sup \frac{|A \cap[1, n]|}{n} .
$$

Informalmente, $\bar{d}$ determina a fração de $\mathbb{N}$ ocupada pelo conjunto $A$, por exemplo, a densidade do conjunto dos números pares (e dos ímpares) é $\frac{1}{2}$, enquanto a densidade do conjunto dos números múltiplos de 3 é $\frac{1}{3}$.

Em 1936 Erdős e Turán conjecturaram que qualquer conjunto com densidade não nula conteria progressões aritméticas de comprimento arbitrário, uma generalização do Teorema de Van der Waerden. Em 1975 Endre Szemerédi demonstrou que a conjectura é verdadeira, também com um complexo argumento combinatório, estendendo resultados anteriores. Já em 1977 Hillel Furstenberg desenvolve uma demonstração completamente nova, utilizando Teoria Ergódica. Um sistema dinâmico é uma dupla $(X, T)$, em que $X$ é um espaço topológico e $T: X \rightarrow X$ é uma função contínua. Para cada $x \in X$, considere a sequência $\left(T^{n}(x)\right)_{n \in \mathbb{N}}$. Dizemos que um ponto $x \in X$ é recorrente se, para toda vizinhança $V$ de $x$, o conjunto $\left\{n \in \mathbb{N} \mid T^{n}(x) \in V\right\}$ é infinito.

Para o caso em que $X$ é um espaço compacto de Hausdorff, podemos estender a iteração de funções e definir pontos $T^{\mathscr{U}}(x)$, para $\mathscr{U}$ um ultrafiltro, e com isso transformar a questão sobre a existência de pontos recorrentes em uma questão sobre a existência de pontos fixos. Dados um ponto $x \in X$ e um ultrafiltro $\mathscr{U}$ sobre $\mathbb{N}$, definimos $T^{\mathscr{U}}(x) \in X$ como o único ponto satisfazendo 
a propriedade de que, para toda vizinhança $V$ de $T^{\mathscr{U}}(x),\left\{n \in \mathbb{N} \mid T^{n}(x) \in V\right\} \in \mathscr{U}$.

Com isso podemos mostrar que um ponto $x \in X$ é recorrente se, e somente se, existe algum ultrafiltro $\mathscr{U}$ tal que $x=T^{\mathscr{U}}(x)$. Mais do que isso, vimos que os resultados de Teoria de Ramsey dão origem a ultrafiltros cujos elementos tem as propriedades correspondentes ao resultado, como o Teorema de Van der Waerden, que garante a existência de ultrafiltros contendo apenas conjuntos que possuem progressões aritméticas de comprimento arbitrário. Suponha que $\mathscr{U}$ seja um desses ultrafiltros e que $T^{\mathscr{U}}(x)=x$, note que este é um ponto recorrente com a propriedade adicional de, para toda vizinhança $V$ de $x$, o conjunto $\left\{n \in \mathbb{N} \mid T^{n}(x) \in V\right\}$ possuir progressões aritméticas arbitrariamente longas. A ideia de se usar sistemas dinâmicos em Teoria de Ramsey é que em alguns casos podemos fazer o caminho inverso, e a partir das propriedades dos conjuntos de recorrência de um ponto, determinar um ultrafiltro.

A observação fundamental de Furstenberg para desenvolver sua teoria é que a densidade dos naturais se assemelha a uma medida invariante em um sistema dinâmico que preserva medida. De fato, considere $\mathbb{N}$ e a função $S: \mathbb{N} \rightarrow \mathbb{N}$ dada por $S(n)=n+1$, pode-se mostrar que $\bar{d}\left(S^{-1}(A)\right)=$ $\bar{d}(A)$.

Essa relação se dá pelo Princípio da Correspondência de Furstenberg (6.8). Com ele relacionamos à Teoria de Ramsey não apenas as propriedades dos conjuntos de recorrência dos pontos no sistema dinâmico, mas também a medida desses conjuntos.

Assim, para mostrar que todo conjunto de densidade não nula possui progressões aritméticas arbitrariamente longas, mostramos que todo conjunto de medida não nula em um sistema dinâmico que preserva medida possui um ponto cuja recorrência se dá por conjuntos que contém progressões de comprimento arbitrário, e então utilizar o Princípio da Correspondência para concluir o resultado.

A partir de então se desenvolveram generalizações envolvendo densidade para diversos outros resultados, de fato, este é, até hoje, um campo de pesquisa bastante ativo. Esperamos que este trabalho possa servir de porta de entrada para outros alunos e seja uma surpresa positiva, assim como foi para o próprio autor, perceber como estas áreas da matemáticas estão muito mais próximas do que aparentam em um primeiro momento. 
CAPÍTULO

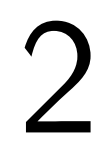

\section{CONSTRUÇÕES BÁSICAS}

Nosso objetivo principal neste capítulo é construir a compactificação de Stone-Čech de semigrupos discretos utilizando ultrafiltros. Para isso precisamos definir os conceitos básicos e demonstrar os resultados preliminares para tal construção.

A compactificação de Stone-Čech nos permite ver um espaço completamente regular como subespaço denso de um espaço topológico compacto de Hausdorff. Além disso, qualquer função contínua poderá ser estendida a esse novo domínio e isso nos permitirá, por exemplo, generalizar o conceito de limite de sequências.

\subsection{Ultrafiltros}

Intuitivamente os filtros definem subconjuntos "grandes" de um espaço e, para isso, ele deve satisfazer algumas condições advindas da nossa concepção intuitiva de grandeza.

A primeira é que o "todo" é "grande". A segunda é que algo que contenha uma coisa "grande" deve ser também "grande". Por fim, a parte comum entre duas coisas "grandes" deve também ser "grande". Formalmente temos a definição de filtro:

Definição 2.1. Um filtro sobre um conjunto $X$ é uma família $\mathscr{F} \subset \mathscr{P}(X)$ de subconjuntos de $X$ satisfazendo:

1. $\emptyset \notin \mathscr{F}$ e $X \in \mathscr{F}$.

2. Se $A \subset B$ e $A \in \mathscr{F}$, então $B \in \mathscr{F}$.

3. Se $A, B \in \mathscr{F}$, então $A \cap B \in \mathscr{F}$.

De todos os subconjuntos de $X$, apenas os que pertencem a $\mathscr{F}$ são considerados grandes. Seguem alguns exemplos: 
Exemplo 2.2. $\quad$ É fácil ver que $\mathscr{F}=\{X\}$ é um filtro, ou seja, o único conjunto grande é o todo.

- Em um espaço de medida, os subconjuntos de medida total formam um filtro.

- Em um espaço topológico, os conjuntos de interior denso formam um filtro.

- Seja $A \subset X$ não vazio. Definimos $\mathscr{F}=\{B \subset X \mid A \subset B\}$. É fácil ver que $\mathscr{F}$ é, de fato, um filtro, mas com uma propriedade adicional, $\bigcap_{B \in \mathscr{F}} B=A \neq \emptyset$.

- Considere $\mathscr{F}=\{A \subset X \mid X \backslash A$ é finito $\}$ em que $X$ é um conjunto infinito. Também é claro que $\mathscr{F}$ satisfaz os axiomas de filtro, porém diferente do caso anterior, temos $\bigcap_{B \in \mathscr{F}} B=\emptyset$.

Definição 2.3. Uma família $\mathscr{A} \subset \mathscr{P}(X)$ tem a propriedade da interseção finita (ou apenas PIF) se para todo subconjunto finito $\left\{A_{1}, \ldots, A_{n}\right\} \subset \mathscr{A}$, ocorrer $\cap_{i=1}^{n} A_{i} \neq \emptyset$.

O item (3) da definição de filtro nos garante que qualquer filtro possui a PIF (por uma indução simples). Mais do que isso, qualquer conjunto com esta propriedade determina unicamente um filtro.

Proposição 2.4. Dado $\mathscr{A} \subset \mathscr{P}(X)$ satisfazendo a PIF, existe um único filtro $\mathscr{F}$ sobre $X$ contendo $\mathscr{A}$ que é minimal com relação à inclusão, ou seja, se $\mathscr{G}$ é um filtro com $\mathscr{A} \subset \mathscr{G}$, então $\mathscr{F} \subset \mathscr{G}$.

Demonstração. Defina

$$
\mathscr{F}=\left\{F \subset X \mid \exists\left\{A_{1}, \ldots, A_{n}\right\} \subset \mathscr{A} \operatorname{com} \bigcap_{i=1}^{n} A_{i} \subset F\right\}
$$

Vamos mostrar que $\mathscr{F}$ é o filtro procurado. É imediato que tal conjunto satisfaz (1) na definição de filtro. $\mathrm{O}$ item (2) também é imediato, pois se $F \in \mathscr{F}$ e $F \subset B$, então existe $\left\{A_{1}, \ldots, A_{n}\right\} \subset \mathscr{A}$ com $\bigcap_{i=1}^{n} A_{i} \subset F \subset B$, portanto $B \in \mathscr{F}$.

Se $F, G \in \mathscr{F}$, então existem $\left\{A_{1}, \ldots, A_{n}\right\} \subset \mathscr{A}$ com $\bigcap_{i=1}^{n} A_{i} \subset F$ e $\left\{B_{1}, \ldots, B_{m}\right\} \subset \mathscr{A}$ com $\bigcap_{i=1}^{m} B_{i} \subset G$. Portanto $\bigcap_{i=1}^{n} A_{i} \cap \bigcap_{i=1}^{m} B_{i} \subset F \cap G$, logo, $F \cap G \in \mathscr{F}$, pois $\left\{A_{1}, \ldots, A_{n}, B_{1}, \ldots, B_{m}\right\}$ é um subconjunto finito de $\mathscr{A}$.

Por fim, se $\mathscr{G}$ é um filtro com $\mathscr{A} \subset \mathscr{G}$, então para todo $F \in \mathscr{F}$ existe $\left\{A_{1}, \ldots, A_{n}\right\} \subset \mathscr{A}$ com $\bigcap_{i=1}^{n} A_{i} \subset F$. Pelo item (3) da definição de filtro, $\bigcap_{i=1}^{n} A_{i} \in \mathscr{G}$, e por (2) temos que $F \in \mathscr{G}$.

Exemplo 2.5. Seja $A \subset X$ um subconjunto não vazio. É claro que o conjunto unitário $\mathscr{A}=\{A\}$ tem a PIF, e o filtro minimal que o estende é $\mathscr{F}=\{B \subset X \mid A \subset B\}$.

Seja $X=\mathbb{N}$ e considere $\mathscr{A}=\left\{2^{n} \mathbb{N} \mid n \in \mathbb{N}\right\}$. Se $A_{1}, \ldots, A_{j} \in \mathscr{A}$, então para cada $i \in\{1, \ldots, j\}$ existe $n_{i} \in \mathbb{N}$ tal que $A_{i}=2^{n_{i}} \mathbb{N}$ e $\bigcap_{i=1}^{j} A_{i}=2^{k} \mathbb{N}$ em que $k=\max \left\{n_{1}, \ldots, n_{j}\right\}$, logo, $\mathscr{A}$ tem a PIF. Neste caso, porém, $\bigcap_{A \in \mathscr{A}} A=\emptyset$, e, diferente dos filtros no exemplo anterior, não existe uma forma explícita de caracterizar seus elementos. 
Como dissemos, podemos interpretar os filtros como subconjuntos grandes de um conjunto, mas ainda podemos pedir a propriedade adicional de que conjuntos "pequenos" quando unidos não podem formar um conjunto grande.

Considere o filtro $\mathscr{F}=\{A \subset \mathbb{N} \mid\{1,2\} \subset A\}$ sobre $\mathbb{N}$, então $\{1\} \cup\{2,3,4, \ldots\}=\mathbb{N} \in \mathscr{F}$, mas nenhum dos dois conjuntos pertencem a $\mathscr{F}$. Na definição de ultrafiltros exigimos que algum dos dois conjuntos pertença a $\mathscr{F}$.

Dissemos que os filtros determinam subconjuntos "grandes", porém os ultrafiltros estendem essa analogia, separando todos os subconjuntos em "grande" ou "pequeno".

Definição 2.6. Uma família $\mathscr{U} \subset \mathscr{P}(X)$ é um ultrafiltro se $\mathscr{U}$ é um filtro satisfazendo a seguinte condição adicional:

4. Para todo $A \subset X, A \in \mathscr{U}$ ou $X \backslash A \in \mathscr{U}$.

Dependendo da situação será mais conveniente utilizar alguma das equivalências apresentadas no resultado à seguir.

Proposição 2.7. Seja $\mathscr{U}$ um filtro. São equivalentes:

1. $\mathscr{U}$ é ultrafiltro.

2. Se $A \cup B \in \mathscr{U}$, então $A \in \mathscr{U}$ ou $B \in \mathscr{U}$.

3. Se $X=A_{1} \cup \cdots \cup A_{n}$, então existe $1 \leq k \leq n \operatorname{com} A_{k} \in \mathscr{U}$.

4. Se $X=A_{1} \cup \cdots \cup A_{n}$ e $A_{i} \cap A_{j}=\emptyset, \forall i \neq j$, então existe um único $1 \leq k \leq n \operatorname{com} A_{k} \in \mathscr{U}$.

5. $\mathscr{U}$ é filtro maximal, ou seja, não existe filtro contendo $\mathscr{U}$ propriamente.

Demonstração. $1 \Rightarrow 2$ ) Como $\mathscr{U}$ é ultrafiltro, temos que $A \in \mathscr{U}$ ou $X \backslash A \in \mathscr{U}$. Se $A \in \mathscr{U}$ acabamos, vamos supor então que $X \backslash A \in \mathscr{U}$. Como $A \cup B \in \mathscr{U}$, então $(X \backslash A) \cap(A \cup B) \in \mathscr{U}$, mas $(X \backslash A) \cap(A \cup B) \subset B$, portanto $B \in \mathscr{U}$.

$2 \Rightarrow 3$ ) Aplique indução sobre $n$. O item (2) é o caso base.

$3 \Rightarrow 4)$ A existência é garantida pelo item (3). Suponha que existam dois conjuntos $A_{i}, A_{j} \in \mathscr{U}$, então $A_{i} \cap A_{j}=\emptyset \in \mathscr{U}$, contradição.

$4 \Rightarrow 5$ ) Seja $\mathscr{V}$ um ultrafiltro tal que $\mathscr{U} \subset \mathscr{V}$ e considere $A \in \mathscr{V}$. Como $\mathscr{U}$ é ultrafiltro, temos que $A \in \mathscr{U}$ ou $X \backslash A \in \mathscr{U}$, mas $X \backslash A \notin \mathscr{V}$ e $\mathscr{U} \subset \mathscr{V}$, então $A \in \mathscr{U}$.

$5 \Rightarrow 1)$ Seja $A \subset X$ e suponha que $A \notin \mathscr{U}$. Afirmamos que existe $B \in \mathscr{U}$ tal que $B \cap A=\emptyset$.

De fato, suponha que por contradição que $A \cap B \neq \emptyset$ para todo $B \in \mathscr{U}$, então $\mathscr{U} \cup\{A\}$ tem a propriedade da interseção finita, e pela Proposição 2.4 existe um filtro contendo $\mathscr{U} \cup\{A\}$, portanto $\mathscr{U}$ não é maximal.

Concluímos então a existência de um elemento $B \in \mathscr{U} \operatorname{com} A \cap B=\emptyset, \log$ o $B \subset X \backslash A$ e assim $X \backslash A \in \mathscr{U}$. 
Já vimos que uma família com a propriedade da interseção finita pode ser estendida a um filtro. A seguir veremos que todo filtro pode ser estendido a um ultrafiltro.

Para a demonstração utilizaremos o Lema de Zorn, que é equivalente ao Axioma da Escolha. Apresentaremos seu enunciado a seguir sem demonstração. As notas de aula Aurichi (2018) apresentam esse e outros resultado equivalentes ao Axioma da Escolha.

Definição 2.8. Uma conjunto parcialmente ordenado é uma dupla $(P, \leq)$ em que $P$ é um conjunto e $\leq$ é uma relação sobre $P$ satisfazendo:

1. Para todo $p \in P, p \leq p$. (Reflexividade)

2. Para todo $p, q \in P$, se $p \leq q$ e $q \leq p$, então $p=q$. (Antissimetria)

3. Para todo $p, q, r \in P$, se $p \leq q$ e $q \leq r$, então $p \leq r$. (Transitividade)

Dizemos também que $\leq$ é uma ordem parcial sobre $P$.

Uma cadeia em $P$ é uma família $\left\{p_{\xi}\right\}_{\xi<\kappa}, \kappa$ um cardinal qualquer, com $p_{\alpha} \leq p_{\beta}$ para todo $\alpha \leq \beta$.

Exemplo 2.9. - Seja $X$ um conjunto, então $(\mathscr{P}(X), \subset)$ é um conjunto parcialmente ordenado.

- Sejam $(P, \leq)$ um conjunto parcialmente ordenado e $Q \subset P$, então $(Q, \leq)$ é também um conjunto parcialmente ordenado.

Lema 2.10 (Zorn). Seja $(P, \leq)$ um conjunto parcialmente ordenado. Se para toda cadeia $Q \subset P$ existe $p \in P \operatorname{com} q \leq p$ para todo $q \in Q$, ou seja, toda cadeia de $P$ admite majorante, então existe um elemento maximal em $P$.

Uma observação para o leitor interessado em Teoria dos Conjuntos: o teorema a seguir é conhecido como o Lema do Ultrafiltro. Apesar de em sua demonstração utilizarmos fortemente o Axioma da Escolha, na forma do Lema de Zorn, pode-se mostrar que este ele é estritamente mais fraco do que o Axioma da Escolha, ou seja, existe um modelo satisfazendo ZF mais o Lema do Ultrafiltro em que o Axioma da Escolha não vale. Portanto neste ponto ainda não estamos utilizando totalmente a força dos axiomas de ZFC. Mais sobre o assunto pode ser encontrado no livro Jech (1973).

Teorema 2.11. Seja $X$ um conjunto. Para todo filtro $\mathscr{F}$ sobre $X$, existe um ultrafiltro $\mathscr{U}$ com $\mathscr{F} \subset \mathscr{U}$.

Demonstração. Considere o conjunto $\{\mathscr{G} \mid \mathscr{G}$ é um filtro e $\mathscr{F} \subset \mathscr{G}\}$ e sobre ele considere a ordem da inclusão. Afirmamos que a união de uma cadeia crescente de filtros é ainda um filtro.

Considere uma família $\left\{H_{\xi}\right\}_{\xi<\kappa}$ de filtros sobre $X \operatorname{com} H_{\alpha} \subset H_{\beta}$ para $\alpha<\beta$ e defina $H=$ 
$\bigcup_{\xi<\kappa} H_{\xi}$. Por definição temos que $X \in H_{\xi}$ e $\emptyset \notin H_{\xi}$ para todo $\xi<\kappa$, portanto $X \in H$ e $\emptyset \notin H$. Se $A, B \in H$, então existem $\alpha, \beta<\kappa \operatorname{com} A \in H_{\alpha}$ e $B \in H_{\beta}$, logo, se $\gamma=\max \{\alpha, \beta\}$, então $A, B \in H_{\gamma}$, portanto $A \cap B \in H_{\gamma}$ e assim $A \cap B \in H$.

Por fim, se $A \in H$ e $A \subset B$, então existe $\alpha<\kappa \operatorname{com} A \in H_{\alpha}$ e $B \in H_{\alpha}$, pois $H_{\alpha}$ é filtro, portanto $B \in H$.

Com isso mostramos que estamos nas condições do Lema de Zorn, assim, existe um filtro $\mathscr{U} \in\{\mathscr{G} \mid \mathscr{G}$ é um filtro e $\mathscr{F} \subset \mathscr{G}\}$ maximal. Pelo teorema anterior, $\mathscr{U}$ é ultrafiltro.

Definição 2.12. Seja $\mathscr{U}$ um ultrafiltro. Temos duas possibilidades: $\bigcap_{A \in \mathscr{U}} A \neq \emptyset$ ou $\bigcap_{A \in \mathscr{U}} A=\emptyset$. No primeiro caso dizemos que $\mathscr{U}$ é um ultrafiltro principal, no segundo caso dizemos que $\mathscr{U}$ é um ultrafiltro livre, ou simplesmente não principal.

Exemplo 2.13. Analisaremos a diferença entre filtros e ultrafiltros a partir dos exemplos em 2.2

- Seja $X$ um conjunto e $\mathscr{F}=\{X\}$. Se $X$ não for um conjunto unitário, então para todo $x \in X, X \backslash\{x\} \neq \emptyset$ e $X \neq\{x\}$. Como $\{x\} \notin \mathscr{F}$ e $X \backslash\{x\} \notin \mathscr{F}$, concluímos que $\mathscr{F}$ não é um ultrafiltro.

- Seja $(X, \mathscr{B}, \mu)$ um espaço de medida finita e suponha que exista um conjunto $A \in \mathscr{B}$ com $0<\mu(A)<\mu(X)$, então a família dos conjuntos de medida total não é um ultrafiltro, pois $\mu(A)<\mu(X)$ e $\mu(X \backslash A)<\mu(X)$. Caso $\mu$ seja uma medida com imagem $\{0,1\}$, então a família dos conjuntos de medida total é um ultrafiltro.

- Em um espaço topológico $X$, se existir um aberto $A \subset X$ que não seja denso, então os conjuntos de interior denso formam um filtro, mas não um ultrafiltro, pois $A$ e $X \backslash A$ não tem interior denso.

- Seja $A \subset X$ não vazio. Então $\mathscr{F}=\{B \subset X \mid A \subset B\}$ se, e somente se, $A$ é um conjunto unitário.

- Considere $\mathscr{F}=\{A \subset X \mid X \backslash A$ é finito $\}$ em que $X$ é um conjunto infinito. Particione $X$ em dois conjuntos infinitos $A$ e $B$, então $A \notin \mathscr{F}$ e $B \notin \mathscr{F}$, portanto $\mathscr{F}$ é um filtro, mas não um ultrafiltro.

Para cada $x \in X$, denotaremos por $\hat{x}$ o ultrafiltro $\{A \subset X \mid x \in A\}$.

É claro todo $x \in X$ determina o ultrafiltro principal $\hat{x}$, mas o próximo resultado diz que esses são os únicos ultrafiltros principais, logo, temos uma bijeção entre elementos de $X$ e os ultrafiltros principais.

Proposição 2.14. Seja $X$ um conjunto. Um ultrafiltro $\mathscr{U}$ é principal se, e somente se, $\mathscr{U}=\hat{x}$ para algum $x \in X$.

Demonstração. Seja $\mathscr{U}$ um ultrafiltro principal e defina $U=\bigcap_{A \in \mathscr{U}} A \neq \emptyset$.

Note que $U \in \mathscr{U}$, pois caso contrário $\mathscr{U} \cup\{U\}$ teria a PIF, e assim poderia ser estendido a um 
ultrafiltro $\mathscr{V} \operatorname{com} \mathscr{U} \subset \mathscr{U} \cup\{U\} \subset \mathscr{V}$, contrariando a maximalidade de $\mathscr{U}$.

Suponha que por contradição que $U$ não seja um conjunto unitário, então existe $x \in U$ tal que $U \backslash\{x\} \neq \emptyset$. Pela Proposição 2.7, item (2), temos que $\{x\} \in \mathscr{U}$ ou $U \backslash\{x\} \in \mathscr{U}$, mas ambas as possibilidades contradizem a suposição de que $U=\bigcap_{A \in \mathscr{U}} A$.

Com isso concluímos que existe $x \in X$ tal que $\{x\}=U \in \mathscr{U}$.

Por fim, seja um conjunto $A \subset X$ qualquer, temos duas possibilidades: $x \in A$, e neste caso $\{x\} \subset A$, $\operatorname{logo} A \in \mathscr{U}$; ou $x \notin A$, e então $\{x\} \cap A=\emptyset$, portanto $A \notin \mathscr{U}$. Assim, $\mathscr{U}=\{A \subset X \mid x \in A\}$.

A recíproca é imediata, pois se $\mathscr{U}=\hat{x}$, então para todo $A \in \mathscr{U}$, temos que $x \in A, \log , x \in$ $\bigcap_{A \in \mathscr{U}} A$.

Corolário 2.15. Um ultrafiltro $\mathscr{U}$ é principal se, e somente se, existe algum $A \in \mathscr{U}$ finito.

Demonstração. Se $\mathscr{U}$ é principal, pela proposição anterior, existe $x \in X \operatorname{com} \mathscr{U}=\hat{x}$, portanto $\{x\} \in \mathscr{U}$.

Reciprocamente, suponha que $A=\left\{a_{1}, \ldots, a_{n}\right\} \in \mathscr{U}$. Note que $X=X \backslash A \cup \bigcup_{i=1}^{n}\left\{a_{i}\right\}$, logo, pelo item (4) na Proposição 2.7, concluímos que existe algum $a \in\left\{a_{1}, \ldots, a_{n}\right\}$ com $\{a\} \in \mathscr{U}$ (note que $X \backslash A \notin \mathscr{U}$, pois $A \in \mathscr{U}$ e $A \cap X \backslash A=\emptyset$ ), portanto $\mathscr{U}=\hat{a}$.

Corolário 2.16. Um ultrafiltro $\mathscr{U}$ sobre $X$ é livre se, e somente se, contém o filtro dos conjuntos cofinitos.

Demonstração. Seja $\mathscr{U}$ um ultrafiltro não principal e considere um conjunto cofinito $A \subset X$. Pela proposição anterior temos que $X \backslash A \notin \mathscr{U}$, então pela definição de ultrafiltro temos $A \in \mathscr{U}$.

Corolário 2.17. Um conjunto $X$ é infinito se, e somente se, existe um ultrafiltro não principal sobre $X$.

Demonstração. Suponha $X$ infinito. Considere um ultrafiltro $\mathscr{U}$ contendo o filtro dos conjuntos cofinitos de $X$ (existe pelo Teorema 2.11). Note que para todo $x \in X, X \backslash\{x\} \in \mathscr{U}$, portanto $\{x\} \notin \mathscr{U}, \operatorname{logo}, \mathscr{U}$ não é ultrafiltro principal.

Se existir um ultrafiltro não principal $\mathscr{U}$, temos pelo Corolário 2.15 que $\mathscr{U}$ contém somente conjuntos infinitos, portanto $X$ é infinito.

\subsection{Compactificação de Stone-Čech $\beta X$}

Nesta seção vamos mostrar que, quando $X$ é um espaço topológico discreto, podemos definir uma topologia sobre o conjunto dos ultrafiltros de $X$ de modo que possamos encarar $X$ como um subespaço denso do conjunto de ultrafiltros.

Nosso objetivo é utilizar esta construção para estender continuamente a operação de um semigrupo discreto para o espaço de ultrafiltros sobre ele, o que gerará um novo semigrupo.

Neste trabalho nos restringiremos à Compactificação de Stone-Čech de espaços discretos, mas 
para um tratamento mais geral sobre o assunto, contendo inclusive a teoria no contexto de Teoria de Categorias, o leitor pode consultar Walker (1974).

Definição 2.18. Dados dois espaços topológicos $X$ e $Y$, dizemos que a função $i: X \rightarrow Y$ é uma inclusão de $X$ em $Y$ se $i$ define um homeomorfismo entre $X$ e $\operatorname{Im}(i)$, considerando sobre $\operatorname{Im}(i)$ a topologia induzida por $Y$.

Considere $Y$ um espaço topológico e seja $X \subset Y$. É claro que a função $i: X \rightarrow Y$ dada por $i(x)=x$ (ou seja, a identidade restrita a $X$ ) é uma inclusão de $X$ em $Y$.

Considere agora um espaço topológico $Z$ e uma inclusão $j: Z \rightarrow Y$ de modo que $\operatorname{Im}(j)=X$, então $j$ é um homeomorfismo entre $Z$ e $X$. Considere o espaço

$$
Y^{\prime}:=(Y \backslash X) \cup Z
$$

em que os abertos de $Y^{\prime}$ são gerados pelos abertos de $Z$ e pelos abertos de $Y \backslash X$ com a topologia induzida de $Y$. Neste espaço temos que $Z \subset Y^{\prime}$ e $j^{-1}$ é uma inclusão de $X$ em $Y^{\prime}$ com imagem $Z$. É fácil ver que a função $\varphi: Y^{\prime} \rightarrow Y$ dada por $\varphi(y)=y$ se $y \in Y$ e $\varphi(z)=i(z)$ se $z \in Z$ é um homeomorfismo entre $Y^{\prime}$ e $Y$.

Com isso queremos explicitar que se existe uma inclusão de um espaço $Z$ em outro espaço $Y$, então não há prejuízo em considerar que $Z \subset Y$, pois estamos identificando $Z$ com sua imagem. Uma última observação é que sempre que tivermos um inclusão $i$ de um espaço $X$ em outro $Y$, não denotaremos a função como $i: X \rightarrow Y$, mas como $i: X \hookrightarrow Y$.

Definição 2.19. Seja $X$ um espaço topológico completamente regular. Uma compactificação de Stone-Čech de $X$ é um espaço topológico $\beta X$ satisfazendo:

1. Existe uma inclusão $i: X \hookrightarrow \beta X$.

2. A imagem de $i$ é densa em $\beta X(\overline{\operatorname{Im}(i)}=\beta X)$.

3. Seja $Y$ um espaço compacto de Hausdorff. Se $f: X \rightarrow Y$ é contínua, então existe uma única função contínua $\varphi: \beta X \rightarrow Y$ que torna comutativo o seguinte diagrama:

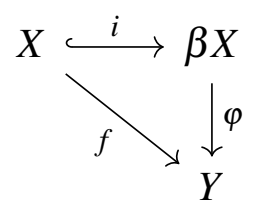

A discussão precedendo esta definição se deve, pois neste trabalho, e em grande parte da literatura sobre o assunto, frequentemente cometeremos o abuso de notação de que $X \subset \beta X$. Pela própria definição podemos concluir que se dois espaços $Y$ e $Z$ são compactificações de Stone-Čech de $X$, então $Y$ e $Z$ são homeomorfos.

De fato, se $i: X \hookrightarrow Y$ é a inclusão de $X$ em $Y$ e $j: X \hookrightarrow Z$ é a inclusão de $X$ em $Z$, então existem as extensões $\varphi: Y \rightarrow Z$ de $i$ e $\psi: Z \rightarrow Y$ de $j$. Construímos o seguinte diagrama: 


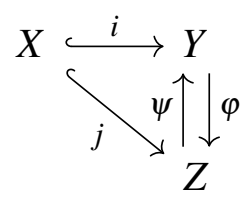

Note que, por definição, $i=\psi \circ j$ e $j=\varphi \circ i$, $\log \circ \varphi \circ \psi \circ j=j$. Considere os dois diagramas abaixo.
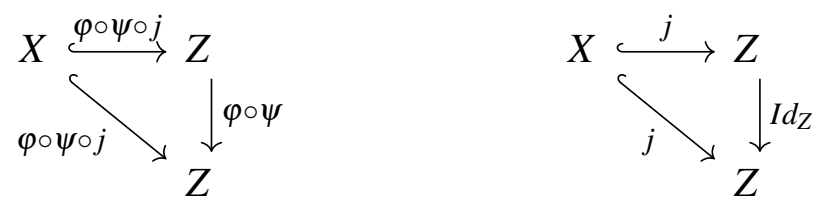

Temos que $(\varphi \circ \psi) \circ(\varphi \circ \psi \circ j)=(\varphi \circ \psi) \circ j$, portanto $\varphi \circ \psi$ é, de fato, a única extensão contínua para o diagrama. Por outro lado, é claro que a identidade em $Z$ é a única extensão contínua para o segundo diagrama, mas como $\varphi \circ \psi \circ j$, concluímos então que os dois diagramas são iguais e, consequentemente, $\varphi \circ \psi$ é a identidade em $Z$.

De forma análoga podemos mostrar que $\psi \circ \varphi$ é a identidade em $Y$, portanto $Y$ e $Z$ são homeomorfos.

Nosso objetivo a partir de agora é definir um topologia sobre o conjunto de ultrafiltros sobre $X$ e mostrar que, caso $X$ seja um espaço discreto, isso determina uma compactificação de Stone-Čech de $X$.

Definição 2.20. Seja $X$ um conjunto. Para cada $A \subset X$, definimos o conjunto

$$
\widehat{A}:=\{\mathscr{U} \in \mathfrak{U} \mid A \in \mathscr{U}\} .
$$

Pela forma como foram definidos e pela definição de ultrafiltro, podemos concluir algumas propriedades desses conjuntos que mostram que eles se comportam de maneira similar aos conjuntos em $X$.

Proposição 2.21. Seja $X$ um conjunto e considere $A, B \subset X$. Temos:

1. Para todo $\mathscr{U} \in \mathfrak{U}, \mathscr{U} \in \widehat{A} \Leftrightarrow A \in \mathscr{U}$.

2. $\widehat{A \cap B}=\widehat{A} \cap \widehat{B}$.

3. $\widehat{A} \cup \widehat{B}=\widehat{A \cap B}$.

4. $\widehat{X}=\mathfrak{U}$.

Demonstração. 1) $\mathscr{U} \in \widehat{A} \Leftrightarrow \mathscr{U} \in\{\mathscr{U} \in \mathfrak{U} \mid A \in \mathscr{U}\} \Leftrightarrow A \in \mathscr{U}$.

2) Pelo item anterior e item 3 da Definição 2.1, temos que

$$
\mathscr{U} \in \widehat{A \cap B} \Leftrightarrow(A \cap B) \in \mathscr{U} \Rightarrow A \in \mathscr{U} \text { e } B \in \mathscr{U} \Leftrightarrow \mathscr{U} \in \widehat{A} \text { e } \mathscr{U} \in \widehat{B} .
$$


3) Pelo item anterior e o item 2 da Proposição 2.7, temos que

$$
\mathscr{U} \in \widehat{A \cup B} \Leftrightarrow(A \cup B) \in \mathscr{U} \Leftrightarrow A \in \mathscr{U} \text { ou } B \in \mathscr{U} \Leftrightarrow \mathscr{U} \in \widehat{A} \text { ou } \mathscr{U} \in \widehat{B} \text {. }
$$

4) Note que para todo ultrafiltro $\mathscr{U}$, temos que $X \in \mathscr{U}$.

Normalmente a topologia sobre um conjunto é muito complexa para ser definida explicitamente, portanto o que faremos é definir uma família de conjuntos mais simples, mas que seja suficiente para definir unicamente uma topologia. Uma família com essa propriedade é dita uma base para uma topologia.

Corolário 2.22. Seja $X$ um conjunto. A família $\{\widehat{A} \subset \mathfrak{U} \mid A \subset X\}$ determina uma base para uma topologia sobre o conjunto de ultrafiltros sobre $X$. Além disso, todos os seus elementos são abertos e fechados.

Demonstração. Seja $\mathfrak{U}$ o conjunto de todos os ultrafiltros sobre $X$ e defina $\mathscr{B}=\{\widehat{A} \subset \mathfrak{U} \mid A \subset X\}$. Primeiro vamos determinar que $\mathscr{B}$ é uma base para uma topologia de $\mathfrak{U}$, para isso precisamos mostrar que os elementos de $\mathscr{B}$ recobrem $\mathfrak{U}$ e que, dados $A, B \in \mathscr{B}$ e $x \in A \cap B$, existe $C \in \mathscr{B}$ com $x \in C \subset A \cap B$.

Pelo item (4) da proposição anterior temos que $\mathfrak{U}=\widehat{X} \in \mathscr{B}$, portanto os elementos de $\mathscr{B}$ recobrem $\mathfrak{U}$.

Dados dois elementos $\widehat{A_{1}} \mathrm{e} \widehat{A_{2}}$ de $\mathscr{B}$, pelo item (2) da proposição anterior temos que $\widehat{A} \cap \widehat{B}=\widehat{A \cap B}$, então dado $\mathscr{U} \in \widehat{A} \cap \widehat{B}, \widehat{A \cap B} \in \mathscr{B}$ e $\mathscr{U} \in \widehat{A \cap B} \subset \widehat{A} \cap \widehat{B}$.

A afirmação de que $\mathscr{B}$ é uma base está, assim, demonstrada.

Para ver que $\mathscr{B}$ é uma base de abertos-fechados, considere um elemento $\widehat{A} \in \mathscr{B}$ qualquer, então $\widehat{X \backslash A} \in \mathscr{B}$ é um aberto, mas $\widehat{A} \cup \widehat{X \backslash A}=\widehat{X}=\mathfrak{U}$ pelos itens (3) e (4) da proposição anterior, portanto $\widehat{A}$ é fechado.

A partir de agora, quando nos referirmos à topologia do espaço de ultrafiltros de um conjunto estaremos nos referindo à topologia gerada por esta base.

Como já vimos anteriormente, existe uma bijeção entre os elementos de $X$ e os ultrafiltros principais sobre $X$, identificando um ponto $x \in X$ com o ultrafiltro $\hat{x} \in \mathfrak{U}$. Além disso, pra todo ultrafiltro principal $\hat{x}$, temos que $\widehat{\{x\}}=\{\hat{x}\}$ é um aberto unitário, logo o conjuntos dos ultrafiltros principais é um espaço discreto (com a topologia induzida).

Suponha que $X$ seja um espaço topológico discreto, então a função $i: X \hookrightarrow \mathfrak{U}$ definida como $i(x)=\hat{x}$ é uma inclusão de $X$ em $\mathfrak{U}$ cuja imagem é o conjunto dos ultrafiltros principais. Assim, podemos identificar um conjunto $A \subset X$, com o conjunto $\{\hat{x} \in \mathfrak{U} \mid x \in A\} \subset \mathfrak{U}$.

A proposição a seguir mostra que podemos considerar o conjunto $\widehat{A}$ como o fecho de $A$ em $\mathfrak{U}$.

Proposição 2.23. Seja $A \subset X$, então $\widehat{A}=\overline{\{\hat{x} \in \mathfrak{U} \mid x \in A\}}$ 
Demonstração. Seja $\mathscr{U} \in \widehat{A}$ e seja $\widehat{B}$ uma vizinhança básica de $\mathscr{U}$ (em que $B \subset X$ ), então existe $x \in A \cap B$ (pois $\mathscr{U}$ é filtro) e, portanto, $\hat{x} \in \widehat{B} \cap \widehat{A}$. Assim, $\widehat{A} \subset \overline{\{\hat{x} \in \mathfrak{U} \mid x \in A\}}$.

Seja $\mathscr{U} \in \overline{\{\hat{x} \in \mathfrak{U} \mid x \in A\}}$ e considere uma vizinhança básica $\widehat{B}$ de $\mathscr{U}$, portanto $B \in \mathscr{U}$. Por definição existe $\hat{x} \in \widehat{B} \operatorname{com} x \in A$, então $x \in A \cap B$. Isso mostra que para todo $B \in \mathscr{U}, A \cap B \neq \emptyset$. Mas então $\mathscr{U} \cup\{A\}$ tem a PIF, e como $\mathscr{U}$ é ultrafiltro, temos que $A \in \mathscr{U}$, caso contrário $\mathscr{U}$ estaria contido propriamente em $\mathscr{U} \cup\{A\}$.

Estamos em condições de demonstrar que $\mathfrak{U}$ com a topologia aqui definida é uma compactificação de Stone-Čech de $X$, mas antes vamos apresentar uma definição e um lema auxiliares, que facilitarão a demonstração.

Definição 2.24. Seja $X$ um espaço topológico. Dizemos que um ultrafiltro $\mathscr{U}$ sobre $X$ converge para $x \in X$ se, para todo aberto $A \subset X \operatorname{com} x \in A, A \in \mathscr{U}$.

Lema 2.25. Seja $\mathscr{U}$ um ultrafiltro sobre um espaço compacto de Hausdorff $X$, então $\mathscr{U}$ converge para um único ponto $x \in X$.

Demonstração. Suponha que $\mathscr{U}$ não seja convergente, então para todo $x \in X$, existe um aberto $A_{x}$ de modo que $x \in A_{x}$ e $A_{x} \notin \mathscr{U}$, portanto o conjunto dos $A_{x}^{\prime} s$ formam uma cobertura, e, por compacidade, existem $x_{1}, \ldots, x_{n}$ de modo que $\cup_{i=1}^{n} A_{x_{i}}=X$. Como $\mathscr{U}$ é ultrafiltro, um dos $A_{x_{i}}$ pertence a $\mathscr{U}$, contradição.

Sejam $x_{1}, x_{2} \in X \operatorname{com} x_{1} \neq x_{2}$. Sendo $X$ Hausdorff, existem abertos disjuntos $A_{1}$ e $A_{2}$ tais que $x_{1} \in A_{1}$ e $x_{2} \in A_{2}$, assim, não podemos ter que ambos os abertos pertencem a $\mathscr{U}$.

Perceba que ultrafiltros diferentes podem convergir para um mesmo ponto, mas um ultrafiltro não converge para dois pontos.

A construção da Compactificação de Stone-Čech feita a seguir não é a mais geral, pois se aplica a apenas a conjuntos discretos, mas isso não nos trará prejuízo uma vez que usaremos essa compactificação em semigrupos que, a princípio, não tem nenhuma topologia definida.

Teorema 2.26. Se X é um espaço topológico discreto, então $\mathfrak{U}$ o espaço de todos os ultrafiltros de X com a topologia definida anteriormente,é uma compactificação de Stone-Čech de X.

Demonstração. Mostraremos os pontos separadamente.

1) $\mathfrak{U}$ é de Hausdorff:

Sejam $\mathscr{U}, \mathscr{V} \in \mathfrak{U} \operatorname{com} \mathscr{U} \neq \mathscr{V}$, então existe $A \in \mathscr{U} \operatorname{com} A \notin \mathscr{V}$. Como $\mathscr{V}$ é ultrafiltro, temos que $X \backslash A \in \mathscr{V}, \log 0 \mathscr{U} \in \widehat{A}, \mathscr{V} \in \widehat{X \backslash A}$ e $\widehat{A} \cap \widehat{X \backslash A}=\emptyset$.

2) $\mathfrak{U}$ é compacto:

Por contradição, suponha que $\mathscr{C}$ seja uma cobertura de $\mathfrak{U}$ por abertos básicos que não admita subcobertura finita. Considere $\mathscr{G} \subset \mathscr{P}(X)$ de modo que $\mathscr{C}=\{\widehat{A} \subset \mathfrak{U} \mid A \in \mathscr{G}\}$ e defina $\mathscr{H}=$ $\{X \backslash A \mid A \in \mathscr{G}\}$. Afirmamos que $\mathscr{H}$ tem a propriedade da interseção finita.

De fato, sejam $X \backslash A_{1}, \ldots, X \backslash A_{n} \in \mathscr{H}$ com $A_{1}, \ldots, A_{n} \in \mathscr{G}$. Note que $\bigcup_{i=1}^{n} A_{i} \neq X$, pois, caso 
contrário, teríamos que $\bigcup_{i=1}^{n} \widehat{A}_{i}=\widehat{\bigcup}_{i=1}^{n} A_{i}=\widehat{X}=\mathfrak{U}$, contrariando a suposição de $\mathscr{C}$ não possuir subcobertura finita. Assim, $\bigcap_{j=1}^{n} X \backslash A_{j}=X \backslash\left(\cup_{j=1}^{n} A_{j}\right) \neq \emptyset$, o que termina a demonstração da afirmação.

Seja $\mathscr{U}$ um ultrafiltro estendendo $\mathscr{H}$, ou seja, $\mathscr{H} \subset \mathscr{U}$. Note que, como $\mathscr{C}$ é cobertura, existe $A \in \mathscr{G} \operatorname{com} A \in \mathscr{U}$, mas $X \backslash A \in \mathscr{H}$ e $\mathscr{H} \subset \mathscr{U}, \log \mathrm{o} A \in \mathscr{U}$ e $X \backslash A \in \mathscr{U}$, contradição.

3) Existe uma inclusão de $X$ em $\mathfrak{U}$ :

Como vimos na discussão precedendo a Proposição 2.23, a função $i: X \hookrightarrow \mathfrak{U}$ definida por $i(x)=\hat{x}$ é uma inclusão.

4) A imagem da inclusão $i: X \rightarrow \mathfrak{U}$ é densa em $\mathfrak{U}$ :

Note que a imagem de $i$ é o conjunto de todos os ultrafiltros principais de $\mathfrak{U}$, logo, pela Proposição 2.23 e pelo item (4) da Proposição 2.21,

$$
\overline{\operatorname{Im}(i)}=\overline{\{\hat{x} \in \mathfrak{U} \mid x \in X\}}=\widehat{X}=\mathfrak{U}
$$

5) Se $Y$ é um espaço compacto de Hausdorff e $f: X \rightarrow Y$ é uma função (contínua, pois $X$ é discreto), então existe uma única função contínua $\varphi: \mathfrak{U} \rightarrow Y$ tal que $\varphi \circ i=f$ :

Para cada $\mathscr{U} \in \mathfrak{U}$, defina $f(\mathscr{U})=\left\{A \subset Y \mid f^{-1}(A) \in \mathscr{U}\right\}$. Vamos mostrar que $f(\mathscr{U})$ é um ultrafiltro sobre $Y$.

É imediato que $Y \in f(\mathscr{U})$ e $\emptyset \notin f(\mathscr{U})$.

Sejam $A, B \in f(\mathscr{U})$, então $f^{-1}(A) \cap f^{-1}(B)=f^{-1}(A \cap B)$, portanto $A \cap B \in f(\mathscr{U})$.

Se $A \subset B \operatorname{com} A \in f(\mathscr{U})$, então

$$
f^{-1}(A) \subset f^{-1}(B) \Rightarrow f^{-1}(B) \in \mathscr{U} \Rightarrow B \in f(\mathscr{U}) .
$$

Seja $A \subset Y$ e suponha que $A \notin f(\mathscr{U})$, portanto

$$
f^{-1}(A) \notin \mathscr{U} \Rightarrow\left(X \backslash f^{-1}(A)\right) \in \mathscr{U} .
$$

Mas $\left(X \backslash f^{-1}(A)\right)=f^{-1}(Y \backslash A)$, portanto $Y \backslash A \in f(\mathscr{U})$. Temos então que $f(\mathscr{U})$ é ultrafiltro.

Como $Y$ é compacto de Hausdorff, pelo lema anterior (2.25), $f(\mathscr{U})$ converge para um único ponto $y_{\mathscr{U}} \in Y$. Definimos então a função $\varphi: \mathfrak{U} \rightarrow Y \operatorname{como} \varphi(\mathscr{U})=y_{\mathscr{U}}$.

Para um ultrafiltro principal $\hat{x}$, temos que o ultrafiltro $f(\hat{x})$ converge para $f(x)$, pois para toda vizinhança $V$ de $f(x)$, temos que $x \in f^{-1}(V)$, portanto $f^{-1}(V) \in \hat{x}$. Assim, para todo $x \in X$,

$$
\varphi \circ i(x)=\varphi(\hat{x})=f(x),
$$

ou seja, $\varphi \circ i=f$.

Vamos mostrar que $\varphi$ é contínua.

Considere um elemento $\mathscr{U} \in \mathfrak{U}$ e um aberto $B \subset Y \operatorname{com} \varphi(\mathscr{U}) \in B$. Como $Y$ é compacto de Hausdorff (portanto regular), existe um aberto $V \subset B \operatorname{com} \varphi(\mathscr{U}) \in V$ e $\bar{V} \subset B$. Defina $A=f^{-1}(\bar{V})$, afirmamos que $A \in \mathscr{U}$.

De fato, suponha que isso não ocorra, então $X \backslash A=f^{-1}(Y \backslash \bar{V}) \in \mathscr{U}$, portanto $Y \backslash \bar{V} \in f(\mathscr{U})$, mas pela definição de $\varphi$, como $\varphi(\mathscr{U}) \in V$ e $V$ é aberto, temos que $V \in f(\mathscr{U})$, o que é uma 
contradição, pois $V \cap(Y \backslash \bar{V})=\emptyset$ e $f(\mathscr{U})$ é um ultrafiltro.

Para concluirmos que $\varphi$ é contínua basta mostrarmos que $\varphi(\widehat{A}) \subset B$.

Seja $\mathscr{V} \in \widehat{A}$ e suponha, por contradição, que $\varphi(\mathscr{V}) \notin B$. Note que $Y \backslash \bar{V}$ é aberto e $\varphi(\mathscr{V}) \in Y \backslash \bar{V}$, portanto, pela definição de $\varphi$, temos que $Y \backslash \bar{V}$, o que contraria a suposição de que $A=f^{-1}(V) \in q$. Concluímos, assim, a demonstração da continuidade de $\varphi$.

Para a unicidade, sejam $\varphi_{1}, \varphi_{2}$ duas funções contínuas de $\mathfrak{U}$ para $Y$ satisfazendo $\varphi_{1} \circ i=\varphi_{2} \circ i=f$. Por absurdo, suponha que exista $\mathscr{U} \in \mathfrak{U}$ tal que $\varphi_{1}(\mathscr{U}) \neq \varphi_{2}(\mathscr{U})$, como $Y$ é um espaço de Hausdorff, existem conjuntos abertos $B_{1}, B_{2} \subset Y$ de modo que $\varphi_{1}(\mathscr{U}) \in B_{1}, \varphi_{2}(\mathscr{U}) \in B_{2}$ e $B_{1} \cap B_{2}=\emptyset \cdot \varphi_{1}^{-1}\left(B_{1}\right) \cap \varphi_{2}^{-1}\left(B_{2}\right)$ é um aberto não vazio de $\mathfrak{U}$, portanto existe um ultrafiltro principal $\hat{x} \in \varphi_{1}^{-1}\left(B_{1}\right) \cap \varphi_{2}^{-1}\left(B_{2}\right)$ (o conjunto dos ultrafiltros principais é denso em $\mathfrak{U}$ ), logo $\varphi_{1}(\hat{x})=f(x)=\varphi_{2}(\hat{x})$, o que implica $f(x) \in B_{1} \cap B_{2}$, uma contradição.

A partir de agora, a menos de menção contrária, denotaremos por $\beta X$ para o espaço dos ultrafiltros sobre $X$ com a topologia que definimos, já que sempre faremos a construção da compactificação de Stone-Čech para espaços discretos e, neste caso, o espaço de ultrafiltros é uma compactificação de Stone-Čech de $X$. Também não faremos distinção entre $X$ e os ultrafiltros principais, já que a identificação $x \leftrightarrow \hat{x}$ é uma inclusão de $X$ em $\beta X$. Com isso em mente, diremos que $X \subset \beta X$ e $\bar{X}=\beta X$.

Um ponto importante da demonstração e que será usado diversas vezes é como definir a extensão de uma função em $X$ para $\beta X$. Se temos uma função contínua $f: X \rightarrow Y \operatorname{com} Y$ compacto de Hausdorff e $\mathscr{U} \in \beta X$, então a extensão contínua $\tilde{f}: \beta X \rightarrow Y$ é dada por $\tilde{f}(\mathscr{U})=y$ de forma que, para toda vizinhança $V$ de $y$, temos que $f^{-1}(V) \in \mathscr{U}$.

\subsection{Compactificação de Semigrupos}

$\mathrm{Na}$ seção anterior vimos que podemos estender um espaço discreto $X$ a um espaço compacto de Hausdorff $\beta X$. Esta construção pode ser utilizada para, a partir de um semigrupo $(S,+)$, construirmos um novo semigrupo cujos elementos são os ultrafiltros sobre $S$ e cuja operação estende a operação de $S$.

Definição 2.27. Um semigrupo é uma dupla $(S,+)$ onde $S$ é um conjunto e $+: S \times S \rightarrow S$ é uma operação associativa.

Para que a compactificação de um semigrupo faça sentido, precisamos definir uma topologia sobre $S$. As construções apresentadas anteriormente supõe semigrupos munidos da topologia discreta e diversos resultados necessitarão fortemente das propriedades dos ultrafiltros em suas demonstrações, portanto assumiremos que todos os semigrupos considerados neste trabalho são espaços discretos, independente de já possuírem uma topologia usualmente associada, como é o caso de $\mathbb{Q}$ ou $\mathbb{R}$. 
Definição 2.28. Seja $(S,+)$ um semigrupo. Para cada $s \in S$, definimos as seguintes funções:

$$
\begin{array}{rlrl}
\lambda_{s}: S & \rightarrow S & \rho_{s}: S & \rightarrow S \\
r & \mapsto s+r & r & \mapsto r+s
\end{array}
$$

A seguir construiremos a extensão da operação + de $S$ para $\beta S$. Partiremos da função $\lambda$ definida acima, mas nada impede de fazermos a mesma construção partindo da função $\rho$. A teoria em ambos os casos é a mesma, com a única diferença que as construções que fizermos à esquerda passarão a ser à direita e vice-versa.

Note que para cada $s \in S, \lambda_{s}: S \rightarrow \beta S$ é continua (lembre-se que $S \subset \beta S$ ) e $\beta S$ é compacto de Hausdorff, portanto admite uma única extensão contínua $\tilde{\lambda_{s}}: \beta S \rightarrow \beta S$. Temos então uma operação intermediária:

$$
\begin{aligned}
*: S & \times \beta S \\
(s, \mathscr{U}) & \mapsto s * \mathscr{U}=\tilde{\lambda}_{s}(\mathscr{U})
\end{aligned}
$$

Novamente, como $S$ é discreto, para cada $\mathscr{U} \in \beta S$, podemos definir a função contínua $f_{\mathscr{U}}: S \rightarrow \beta S$ como $f_{\mathscr{U}}(s)=s * \mathscr{U}=\tilde{\lambda}_{s}(\mathscr{U})$ e, assim, considerar sua extensão contínua $\tilde{f_{\mathscr{U}}}$ : $\beta S \rightarrow \beta S$. Com isso temos a extensão da operação do semigrupo $S$ :

$$
\begin{aligned}
\tilde{+}: & \beta S \times \beta S \\
(\mathscr{U}, \mathscr{V}) & \mapsto \mathscr{U} \tilde{+} \mathscr{V}=\tilde{f_{\mathscr{V}}}(\mathscr{U})
\end{aligned}
$$

Denotaremos a extensão $\tilde{+}$ da operação + do mesmo modo que a operação original, ou seja, $+: \beta S \times \beta S \rightarrow \beta S$, assim como $\lambda_{\mathscr{U}}: \beta S \rightarrow \beta S$ e $\rho_{\mathscr{U}}: \beta S \rightarrow \beta S$.

A função $\rho_{\mathscr{U}}: \beta S \rightarrow \beta S$ nada mais é do que a extensão única extensão contínua da função $f_{\mathscr{U}}$ acima, portanto $\rho_{\mathscr{U}}$ é contínua. Pela forma como definimos a extensão contínua no Teorema 2.26, temos que para todo $\mathscr{U}, \mathscr{V} \in \beta S, \rho_{\mathscr{U}}(\mathscr{V})=\mathscr{V}+\mathscr{U}$ é o único ultrafiltro de $\beta S$ tal que para toda vizinhança básica $\widehat{A}$ de $\mathscr{V}+\mathscr{U}$, ou seja, $A \in \mathscr{V}+\mathscr{U}$, temos que $\rho_{\mathscr{U}}^{-1}(\widehat{A}) \in \mathscr{V}$.

Note que este argumento não é válido para $\lambda_{\mathscr{U}}$, mas se $\mathscr{U}=s \in S$, então $\lambda_{s}(\mathscr{U})$ é apenas a extensão contínua de $\lambda_{s}: S \rightarrow \beta S$, portanto é continua.

Definição 2.29. Seja $A \subset S$ e $x \in S$, então $-x+A=\{s \in S \mid x+s \in A\}$.

Apesar de sabermos que a operação + está definida para todo $\beta S$, não é claro como determinar a soma de dois ultrafiltros. A proposição seguinte nos dá uma maneira, ainda que não muito prática, de operar em $\beta S$.

Proposição 2.30. Para quaisquer ultrafiltros $\mathscr{U}$ e $\mathscr{V}$ sobre um semigrupo $(S,+)$, temos: $\mathscr{U}+\mathscr{V}=\{A \subset S \mid\{x \in S \mid\{y \in S \mid y+x \in A\} \in \mathscr{V}\} \in \mathscr{U}\}=\{A \subset S \mid\{x \in S \mid-x+A \in \mathscr{V}\} \in \mathscr{U}\}$. 
Demonstração. Denote $\mathscr{A}=\{A \subset S \mid\{x \in S \mid\{y \in S \mid y+x \in A\} \in \mathscr{V}\} \in \mathscr{U}\}$. Dado $A \in \mathscr{U}+\mathscr{V}$, temos que $\rho_{\mathscr{V}}^{-1}(\widehat{A}) \in \mathscr{U}$, pois $\mathscr{U}+\mathscr{V}=\rho_{\mathscr{V}}(\mathscr{U}), \log \mathrm{o}$

$$
\begin{aligned}
\rho_{\mathscr{V}}^{-1}(\widehat{A}) \in \mathscr{U} & \Rightarrow & \{x \in S \mid x+\mathscr{V} \in \widehat{A}\} \in \mathscr{U} \\
& \Rightarrow & \left\{x \in S \mid \lambda_{x}^{-1}(\widehat{A}) \in \mathscr{V}\right\} \in \mathscr{U} \\
& \Rightarrow & \{x \in S \mid\{y \in S \mid y+x \in A\} \in \mathscr{V}\} \in \mathscr{U},
\end{aligned}
$$

ou seja, $A \in \mathscr{A}$. Dessa forma concluímos que $\mathscr{U}+\mathscr{V} \subset \mathscr{A}$.

Mas afirmamos que $\mathscr{A}$ tem a propriedade da interseção finita, o que, pela maximalidade de $\mathscr{U}+\mathscr{V}$, nos dará a igualdade.

De fato, sejam $A, B \in \mathscr{A}$, então:

$$
\begin{gathered}
\{x \in S \mid-x+A \in \mathscr{V}\} \in \mathscr{U} \text { e }\{x \in S \mid-x+B \in \mathscr{V}\} \in \mathscr{U} \text {, portanto } \\
\{x \in S \mid-x+A \in \mathscr{V}\} \cap\{x \in S \mid-x+B \in \mathscr{V}\}=\{x \in S \mid-x+A \in \mathscr{V} \mathrm{e}-x+B \in \mathscr{V}\} \in \mathscr{U} .
\end{gathered}
$$

Mas então se $x \in\{x \in S \mid-x+A$ e $-x+B \in \mathscr{V}\}$, então $-x+A \cap-x+B \in \mathscr{V}$, portanto $-x+(A \cap B) \in \mathscr{V}$. Assim,

$$
\begin{aligned}
\{x \in S \mid-x+A \in \mathscr{V}\} \cap\{x \in S \mid-x+B \in \mathscr{V}\} \in \mathscr{U} & \Rightarrow\{x \in S \mid-x+(A \cap B) \in \mathscr{V}\} \in \mathscr{U} \\
& \Rightarrow A \cap B \in \mathscr{A} .
\end{aligned}
$$

Por fim, resta mostrar que $\beta S$ é, de fato, um semigrupo, já que a associatividade não é imediata.

Teorema 2.31. Se $(S,+)$ é um semigrupo, então $(\beta S,+)$ é um semigrupo.

Demonstração. Usando sucessivamente a proposição anterior e o fato de que para todo $\mathscr{U} \in \beta S$, $A \subset S$ e $x \in S$ vale

$-x+\{z \in S \mid-z+A \in \mathscr{U}\}=\{y \in S \mid x+y \in\{z \in S \mid-z+A \in \mathscr{U}\}\}=\{y \in S \mid-(y+x)+A \in \mathscr{U}\}$, temos que para todo $A \subset S$ :

$$
\begin{aligned}
A \in(\mathscr{U}+\mathscr{V})+\mathscr{W} & \Leftrightarrow\{z \in S \mid-z+A \in \mathscr{W}\} \in \mathscr{U}+\mathscr{V} \\
& \Leftrightarrow\{x \in S \mid-x+\{z \in S \mid-z+A \in \mathscr{W}\} \in \mathscr{V}\} \in \mathscr{U} \\
& \Leftrightarrow\{x \in S \mid\{y \in S \mid-(y+x)+A \in \mathscr{W}\} \in \mathscr{V}\} \in \mathscr{U} \\
& \Leftrightarrow\{x \in S \mid\{y \in S \mid-y+(-x+A) \in \mathscr{W}\} \in \mathscr{V}\} \in \mathscr{U} \\
& \Leftrightarrow\{x \in S \mid-x+A \in \mathscr{V}+\mathscr{W}\} \in \mathscr{U} \\
& \Leftrightarrow A \in \mathscr{U}+(\mathscr{V}+\mathscr{W}) .
\end{aligned}
$$

Portanto $(\mathscr{U}+\mathscr{V})+\mathscr{W}=\mathscr{U}+(\mathscr{V}+\mathscr{W})$ 


\section{3}

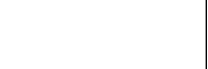

\section{ESTRUTURA ALGÉBRICA DE SEMIGRUPOS}

Dado um semigrupo $(S,+)$ qualquer, construímos um novo semigrupo $(\beta S,+)$ contendo $S$ e estendendo a operação continuamente. Este novo objeto é, em primeiro lugar, um semigrupo, portanto começaremos estudando fatos da estrutura algébrica de semigrupos arbitrários. Mas temos também uma topologia associada a $\beta S$ que é compatível com a operação de semigrupo, assim, aprofundaremos a análise ao considerarmos semigrupos topológicos à direita.

Grande parte dos principais teoremas serão demonstrados a partir da manipulação ou caracterização algébrica de elementos e estruturas de $\beta S$ e, mesmo que por vezes as demonstrações sejam bastante técnicas, serão de grande centralidade neste trabalho e mostram que mesmo um objeto com uma definição tão geral quanto a de semigrupos possui uma estrutura relativamente rígida. É possível o leitor pular este capítulo tendo apenas se familiarizado com as definições básicas de semigrupos (Definição 3.1), o conceito de menor ideal de $S$ (Definição 3.2), e de ordem sobre elementos idempotentes (Definição 3.7). À medida em que forem necessários durante o texto, forneceremos referências aos resultados para que possam ser consultados.

Este capítulo é baseado quase que exclusivamente no livro (HINDMAN; STRAUSS, 2012), um trabalho extensivo e a principal referência sobre o assunto, contendo um estudo bastante aprofundado sobre a álgebra de $\beta S$ e várias de suas aplicações.

\subsection{Semigrupos}

Um semigrupo é uma das estruturas algébricas mais gerais possíveis, uma vez que da operação é exigido apenas a associatividade. Logo todo o trabalho deste capítulo vale também para monóides, grupos e corpos. De modo geral estaremos interessados nos semigrupos $(\mathbb{N},+)$ e $(\beta \mathbb{N},+)$ especificamente, mas utilizaremos os resultados em outros contextos, como em $\mathbb{Z}$ ou no espaço de palavras sobre um alfabeto fixado.

Primeiramente precisamos definir as estruturas algébricas básicas de um semigrupo. 
Definição 3.1. Seja $(S,+)$ um semigrupo:

1. Um conjunto $A \subset S$ não vazio é um subsemigrupo se para todo $a, b \in A, a+b \in A$.

2. Um conjunto $A \subset S$ não vazio é um ideal se para todo $a \in A$ e todo $s \in S, a+s \in A$ e $s+a \in A$. Equivalentemente, $A \subset S$ é ideal se $S+A \subset A$ e $A+S \subset A$. (Em particular, um ideal é um subsemigrupo).

3. Um conjunto $A \subset S$ não vazio é um ideal à esquerda se para todo $a \in A$ e todo $s \in S$, $s+a \in A$. Equivalentemente, $A \subset S$ é ideal se $S+A \subset A$. (Definição análoga para ideal à direita)

4. Um elemento $s \in S$ é idempotente se $s+s=s$.

5. $E(S)=\{s \in S \mid s$ é idempotente).

Seja $\mathscr{I}$ o conjunto de todos os ideais do semigrupo $S$ e suponha que exista um elemento $K \in \mathscr{I}$ minimal.

Seja $I \in \mathscr{I}$ um ideal qualquer. Para todo $k \in K$ e $i \in I$, temos que $i+k \in K \cap I$, em particular, $I \cap K \neq \emptyset$. Além disso, $I \cap K$ é um ideal contido em $K$, portanto, por minimalidade, temos que $K=I \cap K \subset I$. Logo, um ideal minimal é, de fato, o menor ideal de $S$.

Definição 3.2. Denotamos por $K(S)$ o menor ideal de $S$. Se $S$ não admitir ideais próprios, ou seja, $K(S)=S$, então dizemos que $S$ é simples. Dizemos que $S$ é simples à esquerda se não admite ideal à esquerda próprio e definimos analogamente o conceito de simples à direita.

Exemplo 3.3. - Considere o semigrupo $(\mathbb{N},+)$, então, para todo $k \in \mathbb{N}$, o conjunto $\{n \in$ $\mathbb{N} \mid n \geq k\}$ é um ideal. Note que não existe um ideal contido em todos os ideais de $(\mathbb{N},+)$, portanto não existe $K(\mathbb{N})$.

- Considere agora $(\mathbb{N} \cup\{0\}, \cdot)$. Se $I \subset \mathbb{N}$ é um ideal e $i \in I$, então $0 \cdot i=0 \in I$. Por outro lado, $0 \cdot n=0$ para todo $n \in \mathbb{N}$, então $K((\mathbb{N} \cup\{0\}, \cdot))=\{0\}$.

- $\operatorname{Em}(\mathbb{R},+)$ é fácil ver que $\mathbb{R}$ é o único ideal. Portanto $K((\mathbb{R},+))=\mathbb{R}$, ou seja, $(\mathbb{R},+)$ é um semigrupo simples.

Proposição 3.4. Sejam $L$ um ideal à esquerda e $R$ um ideal à direita do semigrupo $(S,+)$, então $L \cap R \neq \emptyset$.

Demonstração. Sejam $a \in L$ e $b \in R$, então $b+a \in L$, pois $S+L \subset L$ e $b+a \in R$, pois $R+S \subset R$. Portanto $b+a \in L \cap R$.

Para todo $t \in S$ o conjunto $S+t$ é um ideal à esquerda de $S$, pois dados $s \in S$ e $a \in S+t$, temos que $a=b+t$ para algum $b \in S$ por definição. Portanto $s+a=s+(b+t)=(s+b)+t \in$ 
$S+t$. De modo análogo construímos o ideal à direita $t+S$ e o ideal $t+S+t$. Mas esses conjuntos possuem uma propriedade adicional caso o elemento $t$ seja idempotente.

Seja $A \subset S$. Um elemento $e \in A$ é uma identidade de $A$ se $e+a=a+e=a$ para todo $a \in A$. De maneira análoga definimos que $e \in A$ é uma identidade à direita de $A$ se $e+a=a$ para todo $a \in A$ e uma identidade à esquerda de $A$ se $a+e=a$ para todo $a \in A$.

Proposição 3.5. Seja $S$ um semigrupo e $e \in E(S)$, então $e$ é identidade à esquerda de $e+S$, identidade à direita de $S+e$ e identidade de $e+S+e$.

Demonstração. Seja $a \in e+S$, então $a=e+b$ para algum $b \in S$, assim, $e+a=e+(e+b)=(e+$ $e)+b=e+b=a$. Invertendo o argumento conseguimos o resultado $S+e$ e consequentemente para $e+S+e$.

Proposição 3.6. Seja $S$ um semigrupo.

1. Um conjunto $L \subset S$ é um ideal à esquerda minimal se, e só se, $S+l=L \forall l \in L$.

2. $I=K(S)$ se, e somente se, $I+s+I=I$ para todo $s \in S$.

Demonstração. 1) Suponha que $L$ seja ideal à esquerda minimal, então dado $l \in L, S+l$ é ideal à esquerda de $S$, mas $S+l \subset S+L \subset L$. Por minimalidade, $S+l=L$.

Reciprocamente, seja $l \in L$, então $L=S+l$ é ideal à esquerda. Tome $J \subset L$ um ideal à esquerda. Se $j \in J$, então $S+j \subset J$, mas $j \in L$, portanto $S+j=L \subset J$.

2) Primeiro suponha $I=K(S)$. Note que $I+s+I$ é ideal para todo $s \in S$, pois $S+(I+s+I)=$ $(S+I)+s+I \subset I+s+I$ e o mesmo vale para $I+s+I+S$. Além disso, $I+s+I=(I+s)+I \subset$ $S+I \subset I$, portanto $I+s+I=I$.

Suponha agora $I+s+I=I$ para todo $s \in S$. Seja $J \subset I$ um ideal e $x \in J$, temos

$$
I=I+x+I=(I+x)+I \subset J+I \subset J .
$$

Portanto $I=J$. Mostramos então que para todo ideal $J \subset I, J=I$, portanto $I$ é um ideal minimal e concluímos que $I=K(S)$.

Definição 3.7. Considere o conjunto $E(S)$ dos elementos idempotentes de $S$. Definimos a relação $\leq$ sobre $E(S)$ como:

$$
e \leq f \text { se, e somente se, } e+f=f+e=e \text {. }
$$

Esta reação, como mostraremos a seguir, é uma ordem parcial sobre o conjunto dos elementos idempotentes, e a partir de agora sempre que nos referirmos a um elemento idempotente minimal, estaremos nos referindo à minimalidade com relação à esta ordem.

Proposição 3.8. A relação $\leq$ definida acima é, de fato, uma ordem parcial. 
Demonstração. Seja um elemento idempotente $e$, então $e+e=e$, o que implica $e \leq_{L} e$.

Sejam dois idempotentes $e$ e $f$ com $e \leq f$ e $f \leq e$, temos $e=e+f=f$.

Por fim, se $e \leq f$ e $f \leq g$, então $e+g=(e+f)+g=e+(f+g)=e+f=e$ e $g+e=$ $g+(f+e)=(g+f)+e=f+e=e$.

Veremos agora que elementos idempotentes estão intimamente relacionados com a estrutura algébrica do semigrupo. Especialmente em $\beta S$, os elementos idempotentes e idempotentes minimais tem propriedades combinatórias importantes, que são centrais ao se estudar a Teoria de Ramsey.

Teorema 3.9. Seja $S$ um semigrupo e seja e $\in E(S)$.

1. Se e pertence a algum ideal à esquerda minimal então e é idempotente minimal.

2. Se S é simples e e é minimal, então $S+e$ é ideal à esquerda minimal.

3. Se todo ideal à esquerda contém um idempotente e e é minimal, então $S+e$ é ideal à esquerda minimal.

Demonstração. (1) Seja $L \subset S$ um ideal à esquerda minimal com $e \in L$. Pelo item (1) da Proposição 3.6, temos que $S+e=L$. Seja $f \in E(S) \operatorname{com} f \leq e$, então $f=f+e \in S+e=L$, assim, $S+f=L$. Como $e$ é identidade à direita em $S+e$ (Proposição 3.5), temos $f=f+e=e$. (2) Suponha que $L \subset S+e$ seja um ideal à esquerda e seja $l \in L$. Como $l \in S+e$, temos que $l=l+e$ pela Proposição 3.5, e como $S$ é simples, $S+l+S=S$, então existem $x, y \in S$ de modo que $x+l+y=e$. Defina $f=e+y+e+x+l$, então

$$
\begin{aligned}
f+f & =(e+y+e+x+l)+(e+y+e+x+l) \\
& =e+y+e+x+(l+e)+y+x+l \\
& =e+y+e+(x+l+y)+x+l \\
& =e+y+(e+e)+x+l \\
& =e+y+e+x+l \\
& =f
\end{aligned}
$$

Portanto $f \in E(S)$. Além disso,

$$
f+e=(e+y+e+x+l)+e=e+y+e+x+(l+e)=e+y+e+x+l=f .
$$

Logo, como $f \leq e$, temos que $f=e$ por minimalidade. Mas $S+e=S+f=S+(e+y+e+x+$ l) $\subset S+l \subset L$, e temos a igualdade.

(3) Suponha que $L \subset S+e$ seja um ideal à esquerda. Seja $f \in L$ idempotente, então $e+f \in L$. Note que $f \in S+e$, portanto $f=f+e$. Assim temos

$$
e+f=e+f+e \text { e } e+f+e+f=e+f+f=e+f
$$


$\log 0 e+f \in E(S)$. Além disso, por minimalidade,

$$
e+(e+f)=e+e+f+e=e+f+e=e+f \Rightarrow e+f \leq e \Rightarrow e+f=e .
$$

Portanto $e \in L \Rightarrow S+e \subset L$.

Em última instância estamos interessados especificamente em resultados sobre a compactificação de Stone-Čech de um semigrupo, logo mesmo que em uma formulação mais geral, veremos que as condições impostas sobre os semigrupos nos resultados seguintes serão satisfeitas por $\beta S$, independente do semigrupo $S$ em questão.

Corolário 3.10. Seja $S$ um semigrupo e $e \in E(S)$. Se $S$ é simples ou todo ideal à esquerda contém um idempotente, então são equivalentes:

1. $e$ é idempotente minimal.

2. $S+e$ é ideal minimal è esquerda.

3. $e$ pertence a algum ideal à esquerda minimal.

Demonstração. A implicação $2 \Rightarrow 3$ é trivial e temos que $3 \Rightarrow 1$ pelo item (1) do teorema anterior.

Se $S$ é simples então $1 \Rightarrow 2$ pelo item (2) do teorema anterior. Se todo ideal à esquerda contém um idempotente, então $1 \Rightarrow 2$ pelo item (3) do teorema anterior.

Lema 3.11. Seja $I \subset S$ um ideal e $L \subset S$ um ideal à esquerda minimal, então $L \subset I$.

Demonstração. Seja $l \in L$. Como $I$ é, em particular, um ideal à direita, existe $m \in I \cap L$ (pela Proposição 3.4), portanto pela minimalidade de $L, S+m=L$. Tome $s \in S$ de modo que $s+m=l$, então $l \in I$, pois $m \in I$.

Lema 3.12. Sejam $S$ um semigrupo e $L \subset S$ um ideal à esquerda minimal. $T \subset S$ é um ideal à esquerda minimal se, e somente se, existe $s \in S$ tal que $T=L+s$. Em outras palavras, dois ideais minimais è esquerda diferem por uma translação.

Demonstração. Seja $T$ um ideal à esquerda minimal. Tome $s \in T$, então $L+s \subset T$ e $S+L+s \subset$ $L+s$. Por minimalidade de $T$, temos $T=L+s$.

Seja $T=L+s . S+L+s \subset L+s$, então resta demonstrar a minimalidade de $T$. Seja $B \subset L+s$ um ideal à esquerda e defina $A=\{l \in L \mid l+s \in B\}$. Note que $A \neq \emptyset$ e $A \subset L$. Seja $a \in A$ e $t \in S$, então $a+s \in B \Rightarrow t+a+s \in B \Rightarrow t+a \in A$, ou seja, $A$ é ideal à esquerda, mas por minimalidade de $L$, temos $A=L$. Assim, $A+s=L+s \subset B \Rightarrow B=L+s$.

Teorema 3.13. Seja $S$ um semigrupo, então

$$
K(S)=\bigcup\{L \subset S \mid L \text { é ideal à esquerda minimal de } S\} .
$$


Demonstração. Seja $I=\bigcup\{L \subset S \mid L$ é ideal à esquerda minimal de $S\}$. Pela Proposição 3.11, se $J$ é ideal de $S$, então $L \subset J$ para todo ideal à esquerda minimal de $S$, portanto $I \subset J$. Assim, resta mostrar que $I$ é um ideal e teremos o resultado.

Sejam $l \in I$ e $s \in S$ e note que $l \in L$ para algum ideal à esquerda minimal $L$ de $S$.

Por um lado, $s+l \in L \subset I$, portanto $I$ é ideal à esquerda. Por outro lado, pela Proposição 3.12, $L+s$ é ideal à esquerda minimal, então $l+s \in L+s \subset I$, portanto $I$ é ideal à direita.

Teorema 3.14. Seja S um semigrupo e suponha que exista um ideal à esquerda minimal que contenha um elemento idempotente. Seja e um idempotente, então são equivalentes:

1. S+e é um ideal à esquerda minimal.

2. $S+e$ é simples à esquerda.

3. $e+S+e$ é um grupo.

4. $e+S$ é um ideal minimal à direita.

5. $e+S$ é simples à direita.

6. e é um idempotente minimal.

7. $e \in K(S)$.

8. $K(S)=S+e+S$.

Demonstração. $1 \Rightarrow 2$ ) Pela definição de minimalidade.

$2 \Rightarrow 3)$ É imediato que $e+S+e$ é fechado pela operação de $S$ e que $e$ é a identidade de $e+S+e$. Resta mostrar que todo elemento de $e+S+e$ possui inverso.

Seja $x=e+s+e \in e+S+e$, em particular, $x \in S+e$. Note que $S+x$ é um ideal à esquerda de $S+e$, e como $S+e$ é simples, temos $S+x=S+e$, portanto $e \in S+x$.

Seja $y \in S$ tal que $e=y+x$, então $e+y+e \in e+S+e$ e $e+y+e+x=y+x=e$ (pois $e$ é a identidade).

$3 \Rightarrow 1)$ Seja $L \subset S+e$ um ideal à esquerda, vamos mostrar que $S+e \subset L$ e assim $S+e$ é ideal à esquerda minimal.

Seja $t \in S+e$, então $e+t \in e+S+e$. Como $e+S+e$ é grupo, existe $-(e+t)$, além disso $-(e+t)+t=-(e+t)+e+t=e \in L$, portanto $S+e \subset S+L \subset L$.

$3 \Rightarrow 4 \Rightarrow 5 \Rightarrow 3$ ) O argumento é idêntico ao das demonstrações anteriores, apenas invertendo os lados.

$1 \Rightarrow 6)$ Seja $f \in S$ um elemento idempotente com $f \leq e$, então $f+e=f \in S+e, \operatorname{logo} S+e=$ $S+f$ e $e \in S+f$. Como $e$ é identidade à direita de $S+e$ e $f$ é identidade à direita de $S+f$, segue que $f=e+f=e$.

$6 \Rightarrow 1)$ Seja $L \subset S+e$ um ideal à esquerda. Basta mostrar que $e \in L$ (assim $S+e \subset L)$.

Seja $t \in L$ idempotente. Note que $(e+t) \in L$ e, como $t \in S+e, t=t+e$, portanto $(e+t)=$ 
$(e+t)+e$, mas então $(e+t)+(e+t)=e+t+t=(e+t)$, ou seja, $(e+t)$ é idempotente. Por outro lado, $e+(e+t)=e+(e+t)+e=(e+t)+e=(e+t)$ e $(e+t)+e=(e+t)$, então $(e+t) \leq e$, e como $e$ é minimal $e=(e+t) \in L$.

$1 \Rightarrow 7)$ Pelo Teorema 3.13, $S+e \subset K(S)$, mas $e \in S+e$.

$7 \Rightarrow 1)$ Se $e \in K(S)$, então pelo Teorema 3.13, existe $L$ um ideal à esquerda minimal com $e \in L$, mas pela Proposição 3.12, $S+e$ é ideal minimal.

$7 \Rightarrow 8)$ Note que $S+e+S$ é um ideal, portanto $K(S) \subset S+e+S$. Porém $e \in K(S)$, então $S+e+S \subset K(S)$ (usando a definição de ideal).

$8 \Rightarrow 7) e=e+e+e \in S+e+S=K(S)$.

\subsection{Semigrupos topológicos à direita}

No capítulo anterior consideramos sobre um semigrupo $S$ a topologia discreta para assim podermos construir a compactificação $\beta S$, portanto $\beta S$ não só possui a estrutura de semigrupo, mas também a de um espaço topológico. Mostraremos que quando a operação de um semigrupo é compatível com sua topologia a existência de algumas estruturas algébricas podem ser garantidas.

Definição 3.15. Um semigrupo topológico à direita é uma tripla $(S,+, \tau)$ de modo que $(S,+)$ é um semigrupo, $(S, \tau)$ é um espaço topológico e $\rho_{s}: S \rightarrow S$ é contínua para todo $s \in S$. Durante todo o trabalho. consideraremos que todos os semigrupos topológicos são de Hausdorff. Neste caso, dizemos que a operação + é contínua à direita.

O conjunto $\Lambda(S)=\left\{s \in S \mid \lambda_{s}\right.$ é contínua $\}$ é chamado o centro topológico de $S$.

Como qualquer função é contínua em um espaço com a topologia discreta, para qualquer semigrupo munido desta topologia temos que $\Lambda(S)=S$. Mas note também que pela construção da extensão da operação de um semigrupo $S$ para $\beta S$ na Seção 2.3, temos que $S \subset \Lambda(\beta S)$.

Por vezes precisaremos verificar se um conjunto $A \subset \beta S$ é um ideal à esquerda, ou seja, que para todo $p \in \beta S, \beta S+p \subset A$. A proposição seguinte usa a compatibilidade entre a operação e a topologia de $\beta S$ para nos permitir determinar o conjunto $\beta S+p$ apenas em termos de $S$.

Proposição 3.16. Para todo $p \in \beta S, \beta S+p=\overline{S+p}$.

Demonstração. Seja $q \in \beta S$ e $V \subset \beta S$ um aberto contendo $q+p$. Como a função $\rho_{p}$ é contínua, $\rho_{p}^{-1}(V)$ é um conjunto aberto, logo existe $s \in S \cap \rho_{p}^{-1}(V)$, pois $S$ é denso em $\beta S$. Segue então que $\rho_{p}(s)=s+p \in V$, portanto $q+p \in \overline{S+p}$, ou seja, $\beta S+p \subset \overline{S+p}$.

Por outro lado, note que $\beta S+p=\rho_{p}(\beta S)$, mas $\beta S$ é compacto e $\rho_{p}$ é contínua, portanto $\beta S+p$ é um conjunto fechado e, consequentemente, fechado. Como $S+p \subset \beta S+p$, temos que $\overline{S+p} \subset \beta S+p$.

O resultado seguinte é de extrema importância, pois garante a existência de ultrafiltros idempotentes na extensão de Stone-Čech de qualquer semigrupo. $\operatorname{Em}(\beta \mathbb{N},+)$, por exemplo, 
não é trivial que exista qualquer elemento idempotente ou que qualquer subsemigrupo fechado possua elemento idempotente. Como corolário imediato temos que qualquer semigrupo finito possui elemento idempotente, já que para semigrupos finitos temos que $S=\beta S$.

Teorema 3.17. Seja $S$ um semigrupo topológico à direita compacto, então $E(S) \neq \emptyset$.

Demonstração. Seja $\mathscr{C}=\{T \subset S \mid T \neq \emptyset, T+T \subset T$ e $T$ é compacto $\}$, ou seja, o conjunto de todos os subsemigrupos compactos não vazios de $S$. Note que $\mathscr{C} \neq \emptyset$, pois $S \in \mathscr{C}$. Com a ordem da inclusão, utilizamos o Lema de Zorn para mostrar que $\mathscr{C}$ possui elemento minimal. De fato, se $\mathscr{A}$ é uma cadeia em $\mathscr{C}$, então $\bigcap \mathscr{A}$ é um limitante inferior para $\mathscr{A}$ (é simples ver que $\bigcap \mathscr{A} \in \mathscr{C}$ ), portanto existe algum elemento $M \in \mathscr{C}$ minimal.

Seja $x \in M$, então $M+x$ é a imagem do conjunto compacto $M$ pela função contínua $\rho_{x}$, portanto $M+x$ é compacto. Além disso, $M+x \subset M+M \subset M$, logo, por minimalidade, $M+x=M$, portanto $M+x \in \mathscr{C}$.

Considere o conjunto $A=\{y \in M \mid y+x=x\}$. Como $M+x=M$, temos que $A \neq \emptyset$. Note que $A=\rho_{x}^{-1}(\{x\})$, portanto $A$ é compacto (pois é fechado), além disso, $A$ é subsemigrupo, já que se $y, z \in A$, então

$$
(y+z)+x=y+(z+x)=y+x=x \Rightarrow(y+z) \in A .
$$

Novamente, por minimalidade, temos $A=M$, portanto $x+x=x$.

Corolário 3.18. Se $S$ é um semigrupo topológico à direita compacto, então todo ideal à esquerda contém um ideal à esquerda minimal e todo ideal à esquerda minimal é fechado (logo, compacto) e contém algum elemento idempotente.

Demonstração. Se $L$ é ideal à esquerda, para todo $l \in L$, temos que $S+l \subset L$ e $S+l$ é ideal à esquerda fechado, pois $S+l=\rho_{l}(S)$ é imagem de um conjunto compacto pela função contínua $\rho_{l}$. Portanto como no teorema anterior, podemos aplicar o Lema de Zorn para conseguir um ideal minimal.

Se $L$ é ideal minimal, então $S+l \subset L$ o que implica $S+l=L$, mas já mostramos que $S+l$ é fechado.

Pela demonstração do teorema anterior temos que qualquer ideal à esquerda compacto contém um idempotente (pois é, em particular, um subsemigrupo), mas todos os ideais minimais são fechados, logo compactos, portanto contém um idempotente.

Note que, em particular, concluímos que todo semigrupo topológico à direita compacto contém um ideal à esquerda minimal, portanto, utilizando o Teorema 3.13, concluímos que, neste caso, existe $K(S)$.

Seja $(S,+)$ um semigrupo topológico à direita compacto e suponha que $S$ possua uma identidade 0 . Se existir algum elemento $e \in E(S)$ diferente da identidade, então $e+0=0+e=e$, portanto $e \leq 0$, assim, 0 é idempotente minimal se, e somente se, $E(S)=\{0\}$. Além disso, se 0 é idempotente minimal, então $S+0=S$ é ideal à esquerda minimal, portanto $S$ é simples à 
esquerda. Por outro lado, se 0 não for o único elemento idempotente de $S$, então existe um idempotente minimal $e \in E(S)$ e $0 \notin S+e$, caso contrário, como $S+e$ é ideal à esquerda minimal, teríamos que 0 seria idempotente minimal.

Dessa forma, um semigrupo topológico à direita compacto possui elemento idempotente diferente da identidade se, e somente se, existe algum ideal à esquerda não trivial.

Proposição 3.19. Sejam $S$ um semigrupo topológico à direita compacto, $L \subset S$ um ideal à esquerda minimal e $R \subset S$ um ideal minimal à direita, então existe $e \in E(S)$ tal que $e \in L \cap R$. Além disso, $L \cap R$ é um grupo.

Demonstração. Pelo corolário anterior existe $f \in L$ idempotente. Pela Proposição 3.6 sabemos que $L=S+f$, pelo Teorema 3.13 temos que $f \in K(S)$ e portanto o Teorema 3.14 garante que $f+S+f$ é um grupo, sendo $f$ sua identidade. Note também que $f+S+f \subset S+f=L$.

Considere $r \in R$ e seja $x \in f+S+f$ o elemento inverso de $f+r+f$, então $x \in L$. Temos que $r+x \in R \cap L, \operatorname{logo} r+x \in K(S)$ pelo Teorema 3.13. Além disso, lembrando que $f$ é identidade de $f+S+f$ :

$$
(r+x)+(r+x)=r+(x+f)+r+(f+x)=r+(x+f+r+f)+x=r+f+x=r+x .
$$

Ou seja, $r+x$ é idempotente. Mas $r+x \in K(S)$ e o Teorema 3.14 implicam $r+x$ ser minimal. Para a segunda parte, defina $e=r+x$. Em primeiro lugar, $e+S+e \subset S+x \subset L$ e $e+S+e \subset$ $r+S \subset R$, portanto $e+S+e \subset L \cap R$. Por outro lado, seja $a \in R \cap L$. Sabemos que $L=S+e$ e $R=e+S, \log 0 a=e+a=a+e$ (Proposição 3.5), portanto $a=e+a+e \in e+S+e$. Concluímos então que $e+S+e=L \cap R$ e pelo Teorema 3.14, $L \cap R$ é um grupo.

Definição 3.20. Seja $(S,+)$ um semigrupo. Um conjunto $A \subset S$ é uma solução à esquerda se existem $s, r \in S$ tais que $A=\{a \in S \mid s+a=r\}$.

$\mathrm{O}$ semigrupo $S$ é dito fracamente cancelativo à esquerda se todas as suas soluções são finitas.

No semigrupo dos naturais temos que $a+s=a+r$ implica $s=r$ para quaisquer $a, s, r \in \mathbb{N}$ (um semigrupo com esta propriedade é dito cancelativo à esquerda). Considere uma solução à esquerda $A \subset \mathbb{N}$. Por definição existem $r, s \in \mathbb{N} \operatorname{com} A=\{a \in S \mid s+a=r\}$. Agora suponha que existam dois elementos $a, b \in A$, então

$$
a, b \in A \Rightarrow s+a=r \text { e } s+b=r \Rightarrow s+a=s+b \Rightarrow a=b .
$$

Em particular, $\mathbb{N}$ é fracamente cancelativo.

Os exemplos naturais que temos de semigrupo são geralmente cancelativos ao menos em um dos lados, mas temos dois exemplos simples para atestar a diferença entre as definições.

Exemplo 3.21. Considere o semigrupo $(\mathbb{N}, \vee), \operatorname{com} m \vee n=\max \{m, n\}$. Seja $A \subset \mathbb{N}$ uma solução à esquerda, então existem $n, m \in \mathbb{N} \operatorname{com} A=\{a \in \mathbb{N} \mid m \vee a=n\}$, mas então $A \subset\{1,2, \ldots, n\}$, portanto é finita. Por outro lado, $2 \vee 3=3=1 \vee 3$, mas $2 \neq 1$, então ( $\mathbb{N}, \vee$ ) é fracamente 
cancelativo, mas não é cancelativo.

Considere agora o semigrupo $(\mathbb{N}, \wedge)$, com $m \wedge n=\min \{m, n\}$. O conjunto $A=\{a \in \mathbb{N} \mid 1 \wedge a=$ $1\}$ é uma solução à esquerda, por definição, mas $A=\mathbb{N}$. Portanto $(\mathbb{N}, \wedge)$ não é fracamente cancelativo.

Seguindo a notação padrão, denotaremos por $S^{*}$ o conjunto dos ultrafiltros não principais de $\beta S$, ou seja, $S^{*}=\beta S \backslash S$.

Proposição 3.22. Um semigrupo infinito $(S,+)$ é fracamente cancelativo à esquerda se, e só se, $S^{*}$ é um ideal à esquerda de $(\beta S,+)$.

Demonstração. Suponha $S$ fracamente cancelativo à esquerda e seja $p \in S^{*}$ (existe pois $S$ é infinito). Seja $q \in \beta S$ e suponha por absurdo que $q+p=r \in S$.

Então $\{r\} \in q+p, \operatorname{logo},\{s \in S \mid-s+\{r\} \in p\} \in q$. Tome então $t \in\{s \in S \mid-s+\{a\} \in p\}$. Isso implica $\{a \in S \mid t+a=s\} \in p$, mas esse conjunto é finito, contrariando $p$ ser ultrafiltro não principal.

Reciprocamente, seja $S^{*}$ um ideal à esquerda e, novamente, suponha por absurdo que existam $r, t \in S$ de modo que $A=\{a \in S \mid t+a=r\}$ seja infinito, então existe um ultrafiltro não principal $p \in S^{*} \operatorname{com} A \in p$.

Note que $t \in S$, portanto $\lambda_{t}$ é uma função contínua, e que $A=\lambda_{t}^{-1}(\{r\})$ é um aberto (pois $r \in S$ ) contendo $p$.

Para toda vizinhança $V$ de $r$, temos que $A \subset \lambda_{t}^{-1}(V)$, logo $\lambda_{t}^{-1}(V)$ é uma vizinhança de $p$. Assim, $\lambda_{t}(p)=t+p=r$ (lembre-se de como definimos a extensão de $S$ para $\beta S$ ), contrariando a hipótese de $S^{*}$ ser ideal à esquerda.

Como corolário temos que se $S$ é fracamente cancelativo à esquerda, não só garantimos a existência de um elemento idempotente de $\beta S$ que não é a identidade do semigrupo, mas a de um elemento idempotente novo, que não pertencia ao semigrupo original. Isso significa que a compactificação de Stone-Čech de qualquer semigrupo fracamente cancelativo à esquerda produz novos elementos idempotentes.

Corolário 3.23. Se $(S,+)$ é um semigrupo infinito fracamente cancelativo à esquerda, então existe $p \in S^{*}$ idempotente.

Demonstração. Basta usar o teorema anterior e o Corolário 3.18.

Proposição 3.24. Seja $S$ um semigrupo topológico à direita compacto, então todos os seus ideias minimais à esquerda são homeomorfos.

Demonstração. Sejam $L$ e $I$ dois ideais à esquerda minimais de $S$ e fixe $z \in I$. Note que $I=S+z$ e existe um idempotente $e \in L$ tal que $L=S+e$. Afirmamos que a função $\varphi=\left.\rho_{z}\right|_{L}$ é o homeomorfismo procurado. 
A função $\varphi$ é contínua por definição de semigrupo topológico à direita. É claro que o domínio de $\varphi$ é $L$ e, para qualquer $s \in L$, temos que $\varphi(s)=s+z \in S+z=I$, portanto o codomínio de $\varphi$ está contido em $I$. Além disso, $\varphi(L)=\varphi(S+e)=S+e+z$ é um ideal à esquerda contido em $I$, logo, por minimalidade, $\varphi(L)=I$, e temos a sobrejetividade. Resta mostrar que $\varphi$ é injetiva.

Pelo Teorema 3.14 sabemos que $e+S+e$ é um grupo e defina $w \in e+S+e$ como o inverso do elemento $e+z+e$ e lembre-se que $z+e=e+z=z$, pois $e$ é uma identidade deste grupo. Seja $s \in L$ qualquer, então

$$
\rho_{w}(\varphi(s))=s+z+w=s+(e+z+e)+w=s+e=s .
$$

A última igualdade segue do fato de $e$ ser uma identidade à direita em $S+e$. Provamos então que $\varphi: I \rightarrow L$ é uma bijeção contínua. Como $S$ é compacto, temos o resultado.

Proposição 3.25. Seja $S$ um semigrupo topológico à direita compacto e seja $A$ um subsemigrupo fechado de $S$ tal que $K(S) \cap A \neq \emptyset$, então $K(A)=A \cap K(S)$.

Demonstração. Em primeiro lugar note que $K(S) \cap A$ é um ideal de $A$. De fato, seja $a \in A$ e $s \in K(S) \cap A$, então $a+s \in A$ pois $A$ é semigrupo e $a+s \in K(S)$ por ser ideal. Pelo Teorema 3.13 temos que $A \cap K(S) \subset K(A)$.

Para a outra inclusão tome $x \in K(A)$, então $A+x$ é um ideal à esquerda de $A$. Mas então pelo Corolário 3.18, $A+x$ contém um elemento idempotente $e$, portanto pela Proposição 3.6, $A+e$ é ideal minimal de $A$. Como $x \in K(S), x \in L$ sendo $L$ um ideal à esquerda minimal de $S$, logo $L=S+x$ e $e \in A+x \subset S+x$, portanto $L=S+e$. Como $e$ é uma identidade à direita de $S+e$ (Proposição 3.5), então $x+e=x$, mas $x+e \in A+e \subset K(A)$.

Por fim, nas demostrações dos teoremas de Van der Waerden e de Halles-Jewett, precisaremos do produto de semigrupos topológicos à direita compactos.

Dada uma família de semigrupos topológicos à direita compactos $\left(S_{i},+_{i}\right)_{i \in I}$, podemos definir o espaço $S=\times_{i \in I} S_{i}$ com a topologia produto. Em analogia com a notação em espaços vetoriais, denotaremos um elemento deste espaço por $\vec{s}$, ou seja, para cada $i \in I$ existe $s_{i} \in S_{i}$ de modo que $\vec{s}=\left(s_{i}\right)_{i \in I}$.

Definimos sobre $S$ a operação $+: S \times S \rightarrow S$ como

$$
\left(s_{i}\right)_{i \in I}+\left(r_{i}\right)_{i \in I}=\left(s_{i}+{ }_{i} r_{i}\right)_{i \in I}
$$

Como as operações $+_{i}$ são associativas em seus respectivos semigrupos, concluímos que a operação + é associativa, logo $(S,+)$ é, de fato, um semigrupo.

O Teorema de Tychonoff garante que o produto de espaços compactos é compacto e, além disso, temos que uma função nele é contínua se, e somente se, todas as suas projeções são contínuas. Logo, como para cada $S_{i}$ a função $\rho_{i, s}: S_{i} \rightarrow S_{i}$ é contínua para todo $s \in S_{i}$, temos que $\rho_{\vec{s}}: S \rightarrow S$ é contínua para todo $\vec{s} \in S$, então $S$ é um semigrupo topológico à direita compacto. Pelo mesmo motivo temos que $\Lambda(S)=\times_{i \in I} \Lambda\left(S_{i}\right)$. 
Proposição 3.26. Seja $\left(S_{i}\right)_{i \in I}$ uma família de semigrupos e seja $S=\chi_{i \in I} S_{i}$, então $K(S)=$ $\chi_{i \in I} K\left(S_{i}\right)$.

Demonstração. Pela Proposição 3.6, temos que para todo $\vec{s} \in S$

$$
\underset{i \in I}{\chi} K\left(S_{i}\right)+\vec{s}+\underset{i \in I}{X} K\left(S_{i}\right)=\underset{i \in I}{\chi}\left(K\left(S_{i}\right)+{ }_{i} s_{i}+{ }_{i} K\left(S_{i}\right)\right)=\underset{i \in I}{X} K\left(S_{i}\right)
$$

Novamente pela mesma proposição, concluímos que $\times_{i \in I} K\left(S_{i}\right)=K(S)$.

De modo geral, a compactificação do produto de semigrupos não é igual ao produto das compactificações deste semigrupo: no caso dos naturais, por exemplo, o que estamos dizendo é que $\beta(\mathbb{N} \times \mathbb{N}) \neq \beta \mathbb{N} \times \beta \mathbb{N}$.

Como definimos a topologia discreta sobre $\mathbb{N}, \mathbb{N} \times \mathbb{N}$ também tem a topologia discreta, portanto a função $\delta: \mathbb{N} \times \mathbb{N} \rightarrow\{0,1\}$ dada por

$$
\delta((m, n))=\left\{\begin{array}{l}
1, \text { se } m=n \\
0, \text { se } m \neq n
\end{array}\right.
$$

é contínua. Suponha, por absurdo, que $\beta \mathbb{N} \times \beta \mathbb{N}$ seja a compactificação de Stone-Čech de $\mathbb{N} \times \mathbb{N}$, então a função $\delta$ admite uma extensão contínua $\tilde{\delta}: \beta \mathbb{N} \times \beta \mathbb{N} \rightarrow\{0,1\}$.

Seja $p \in \mathbb{N}^{*}$. Se $\tilde{\delta}((p, p))=1$, então o conjunto $U=\tilde{\delta}^{-1}(\{1\})$ é uma vizinhança de $(p, p)$. Considere um aberto básico $V$ de $\beta \mathbb{N} \times \beta \mathbb{N}$. Existem $A, B \subset \mathbb{N}$, com $p \in \widehat{A}$ e $p \in \widehat{B}$, tais que $V=\widehat{A} \times \widehat{B}$. Como $p$ é ultrafiltro não principal, os conjuntos $A$ e $B$ são infinitos, logo existem $a \in A$ e $b \in B$ de modo que $a \neq b$ e $(a, b) \in V$, o que implica $V \nsubseteq U$. Como $V$ é um aberto básico qualquer, temos que $U$ não é uma vizinhança de $(p, p)$. Concluímos, portanto, que $\tilde{\delta}((p, p)) \neq 1$. Por outro lado, se $\tilde{\delta}((p, p))=0$, tomamos $U=\tilde{\delta}^{-1}(\{0\})$ e um aberto básico $V=\widehat{A} \times \widehat{B}$ contendo $p$. Note que $A \in p$ e $B \in p, \operatorname{logo} A \cap B \in p$ e, em particular, $A \cap B$ é não vazio. Tome $a \in A \cap B$, então $(a, a) \in V$, mas $(a, a) \notin U$. Assim, $V \nsubseteq U$, e, consequentemente, $U$ não é uma vizinhança de $(p, p)$.

Chegamos, assim, a uma contradição, pois concluímos que se $\tilde{\delta}$ é uma extensão contínua de $\delta$, então $\tilde{\delta}((p, p)) \neq 0$ e $\tilde{\delta}((p, p)) \neq 1$. Logo $\beta \mathbb{N} \times \beta \mathbb{N} \neq \beta(\mathbb{N} \times \mathbb{N})$.

Mesmo com uma formulação mais geral, a proposição seguinte tem por intuito mostrar que o produto de compactificações de um semigrupo, mesmo não satisfazendo a definição de compactificação de Stone-Čech, ainda preserva algumas estruturas algébricas.

Proposição 3.27. Sejam $S$ um semigrupo topológico à direita compacto, $T \subset \Lambda(S)$ um subsemigrupo de $S$ e $A$ um subsemigrupo de $T$. Então:

1. $\bar{A}$ é subsemigrupo de $S$.

2. Se $B$ é ideal à esquerda (direita) de $A$, então $\bar{B}$ é ideal à esquerda (direita) de $\bar{B}$. 
Demonstração. 1) Sejam $x, y \in \bar{A}$. Vamos mostrar que para todo aberto $U \subset S \operatorname{com} x+y \in U$, $U \cap A \neq \emptyset$, e assim concluiremos que $x+y \in \bar{A}$, ou seja, $\bar{A}$ é subsemigrupo.

Seja $U \subset S$ um aberto com $x+y \in U$, temos que $\rho_{y}$ é uma função contínua, portanto $\rho_{y}^{-1}(U)$ é um aberto contendo $x$. Como $x \in \bar{A}$, existe $a \in A \cap \rho_{y}^{-1}(U)$ e, portanto, $a+y \in U$.

Note que $a \in A \subset T \subset \Lambda(S)$, logo a função $\lambda_{a}$ é contínua. Novamente, $\lambda_{a}^{-1}(U)$ é um aberto contendo $y$ e $y \in \bar{A}$, assim, existe $b \in A \cap \lambda_{a}^{-1}(U)$, portanto $a+b \in U$. Como $A$ é subsemigrupo, $a+b \in A$.

2) Sejam $x \in \bar{A}$ e $y \in \bar{B}$. Novamente, vamos mostrar que todo aberto de $S$ contendo $x+y$ tem interseção não vazia $\operatorname{com} B$, e assim teremos $x+y \in \bar{B}$.

Seja $U \subset S$ um aberto com $x+y \in U$. Como $\rho_{y}$ é uma função contínua, $\rho_{y}^{-1}(U)$ é um aberto contendo $x$, logo existe $a \in A \cap \rho_{y}^{-1}(U)$ e portanto $a+y \in U$. Como $B \subset T \subset \Lambda(S)$, temos que $\lambda_{a}$ é contínua, e assim, $\lambda_{a}^{-1}(U)$ é um aberto contendo $y$, logo existe $b \in B \cap \lambda_{a}^{-1}(U)$, portanto $a+b \in U$. Como $B$ é ideal à esquerda de $A, a+b \in A$.

A mesma demonstração vale para $A$ sendo um ideal à direita, apenas invertendo a ordem das operações.

\subsection{Alguns problemas em aberto}

Usando a propriedade universal de $\beta \mathbb{N}$ e o fato de que $\mathbb{N}^{*}$ é um conjunto fechado, e portanto compacto, podemos tomar qualquer função $f: \mathbb{N} \rightarrow \mathbb{N}^{*}$ e estendê-la continuamente a uma função $\tilde{f}: \beta \mathbb{N} \rightarrow \mathbb{N}^{*}$. Como a extensão $\tilde{f}$ preserva injetividade (Lema 5.23), temos que se $f$ é injetiva, então $\beta \mathbb{N}$ e $\tilde{f}(\beta \mathbb{N}) \subset \mathbb{N}^{*}$ são homeomorfos, em outras palavras, podemos encontrar uma "cópia" topológica de $\beta \mathbb{N}$ contida em $\mathbb{N}^{*}$. Mas para além da estrutura topológica, estamos interessados também na estrutura algébrica de $\beta \mathbb{N}$, portanto é natural perguntar se existe algum homeomorfismo de $\beta \mathbb{N}$ para algum subconjunto de $\mathbb{N}^{*}$ que seja também um isomorfismo entre semigrupos.

A resposta foi dada por Dona Strauss em Davenport, Hindman e Leader (2000), em que foi demonstrado que se $\varphi: \beta \mathbb{N} \rightarrow \mathbb{N}^{*}$ é um homomorfismo contínuo, então $\operatorname{Im}(\varphi)$ é um conjunto finito e $\left|\varphi\left(\mathbb{N}^{*}\right)\right|=1$, ou seja, todos os ultrafiltros não principais colapsam para um mesmo ponto. Isso já nos garante que não existe nenhum conjunto contido em $\mathbb{N}^{*}$ que tenha a mesma estrutura topológica e algébrica de $\beta \mathbb{N}$. Resta a questão se existe algum homomorfismo contínuo não trivial de $\beta \mathbb{N}$ para $\mathbb{N}^{*}$, e apesar da pergunta parecer estar muito próxima de ser respondida, permanece em aberto há algumas décadas.

Questão 3.28. Existe algum homomorfismo contínuo $\varphi: \beta \mathbb{N} \rightarrow \mathbb{N}^{*} \operatorname{com}|\operatorname{Im}(\varphi)|>1$ ?

Existem ainda duas formulações equivalentes deste problema.

Teorema 3.29. São equivalentes:

1. Existe um homomorfismo não trivial de $\beta \mathbb{N}$ para $\mathbb{N}^{*}$. 
2. Existe um ultrafiltro $p \in \mathbb{N}^{*}$ não idempotente tal que $p+p$ é idempotente.

3. Existe um subsemigrupo finito de $\mathbb{N}^{*}$ contendo um ultrafiltro não idempotente.

Demonstração. $1 \Rightarrow 2)$ Seja $\varphi: \beta \mathbb{N} \rightarrow \mathbb{N}^{*}$ um homomorfismo contínuo e seja $\varphi(p)=q$ para todo $p \in \mathbb{N}^{*}$. Afirmamos que o conjunto $A=\{x \in \mathbb{N} \mid \varphi(x) \neq q\}$ é limitado.

De fato, suponha que não, então existe algum elemento $r \in \mathbb{N}^{*} \operatorname{com} A \in r$, ou seja, $r \in \widehat{A}$. Mas note que $\varphi(\widehat{A})=\widehat{\varphi(A)}$ e $q \notin \widehat{\varphi(A)}$, portanto $\varphi(r) \neq q$, o que é uma contradição.

Considere então $m=\max (A)$ e considere $p=\varphi(m)$, então $p+p=\varphi(m+m)=q$ pela definição de $m$. Pelo mesmo motivo concluímos as outras igualdades.

$2 \Rightarrow 3)$ Seja $q=p+p$, então o conjunto $\{p, q, p+q\}$ é um subsemigrupo. Note que $p+q=$ $q+p=p+p+p$.

$3 \Rightarrow 1)$ Seja $S \subset \mathbb{N}^{*}$ um semigrupo finito e $p \in S$ um elemento não idempotente. Defina a função $f: \mathbb{N} \rightarrow S$ como $f(n)=n p$, o elemento $p$ somado $n$ vezes. Considere $\varphi: \beta \mathbb{N} \rightarrow \mathbb{N}^{*}$ com a extensão contínua de $f$, então $\varphi$ é um homomorfismo pelo Lema 5.22.

No conjunto dos naturais temos tanto a operação de soma quanto o produto, ou seja, podemos considerar dois semigrupos distintos: $(\mathbb{N},+)$ e $(\mathbb{N}, \cdot)$. Podemos proceder como na Seção 2.3 para definir os semigrupos $(\beta \mathbb{N},+)$ e $(\beta \mathbb{N}, \cdot)$.

Em $\mathbb{N}$ temos uma compreensão vasta de como as operações de adição e multiplicação se relacionam, mas em $\beta \mathbb{N}$ a situação não é tão simples, o que gera diversos problemas que ainda estão em aberto.

Para o caso específico em que $n \in \mathbb{N}$ e $p, q \in \beta \mathbb{N}$, temos que $n \cdot(p+q)=(n \cdot p)+(n \cdot q)$ e $(p+q) \cdot n=(p \cdot n)+(n \cdot q)$, ou seja, vale a distributividde (ver Hindman e Bergelson (1990), Lema 1.2). Em Douwen (1991) foi mostrado o seguinte resultado:

Teorema 3.30. $O$ conjunto $\left\{p \in \mathbb{N}^{*} \mid \forall q, r \in \mathbb{N}^{*}, p \cdot(q+r) \neq(p \cdot q)+(p \cdot r) e(q+r) \cdot p \neq\right.$ $(q \cdot p)+(r \cdot p)\}$ tem interior denso em $\mathbb{N}^{*}$.

Portanto a soma e produto em $\mathbb{N}^{*}$ são altamente não distributivas. A questão permanece, porém, se existe algum outro caso em que ela é válida.

Questão 3.31. Existe $p \in \mathbb{N}^{*}$ e $q, r \in \beta \mathbb{N}$ de modo que $p \cdot(q+r)=(p \cdot q)+(p \cdot r)$ ou $(q+r) \cdot p=$ $(q \cdot p)+(r \cdot p) ?$

Os avanços e resultados relacionados a esta questão podem ser encontrados em Hindman e Strauss (2012), capítulo 13, e estão relacionados a uma questão ainda mais impressionante por sua elementaridade, reforçando a dificuldade de se analisar as relações entre as duas operações em $\beta \mathbb{N}$ :

Questão 3.32. Existem $p, q, r, s \in \mathbb{N}^{*}$ de modo que $p+q=r \cdot s$ ? Equivalentemente, existe $q \in\left(\mathbb{N}^{*}+\mathbb{N}^{*}\right) \cap\left(\mathbb{N}^{*} \cdot \mathbb{N}^{*}\right) ?$ 
CAPÍTULO

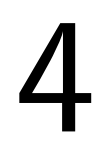

\section{SISTEMAS DINÂMICOS}

Em Blass (1993), Andreas Blass explora como os ultrafiltros sobre os números naturais podem ser relacionados a sistemas dinâmicos $(X, T)$ onde $X$ é um espaço compacto de Hausdorff e $T: X \rightarrow X$ é uma função contínua. O texto se limita, porém, somente ao semigrupo $(\beta \mathbb{N},+)$. O que faremos a seguir é generalizar estes resultados para sistemas dinâmicos sobre semigrupos arbitrários, mostrando que com algumas modificações, os resultados permanecem válidos.

Mostraremos que $\left(\beta S,\left\langle\lambda_{s}\right\rangle_{s \in S}\right)$ não só é um sistema dinâmico universal, mas também contém sistemas dinâmicos minimais universais.

A teoria sobre limites generalizados em sistemas dinâmicos sobre semigrupos arbitrários pode ser encontrada no artigo Hindman, Strauss e Zamboni (2018), que também pode ser consultado para o leitor que quiser aprofundar o estudo sobre as relações entre estruturas algébricas de $\beta S$ e pontos e conjuntos de recorrência de um sistema dinâmico sobre $S$.

\subsection{Limites generalizados}

Em sua forma mais geral, um sistema dinâmico discreto sobre um semigrupo $S$ é apenas um conjunto $X$ e uma família de funções $\left\{T_{s}: X \rightarrow X \mid s \in S\right\}$ satisfazendo a condição de compatibilidade $T_{s+t}=T_{S} \circ T_{t}$ para todo $s, t \in S$. Neste trabalho consideraremos apenas sistemas dinâmicos em que $X$ seja um espaço compacto de Hausdorff e cada uma das funções $T_{S}$ sejam contínuas, com o intuito de utilizar o item (3) da definição de Compactificação de Stone-Čech (Definição 2.19), o que nos permitirá estender a noção de limite.

Usualmente sistemas dinâmicos são denotados por $(X, T)$ pois consideramos a família de funções $\left\{T^{n} \mid n \in \mathbb{N}\right\}$, sendo $T^{n}$ a função $T$ iterada $n$ vezes. Note que isso é um caso especial da nossa definição, tomando $\left(X,\left\langle T^{n}\right\rangle_{n \in \mathbb{N}}\right)$. Neste caso podemos analisar o limite dos pontos $T^{n}(x)$ com $n$ tendendo a infinito, mas para semigrupos arbitrários esse conceito não tem sentido, portanto precisamos do conceito de redes para lidar com este caso.

Apresentaremos aqui apenas o necessário para as aplicações posteriores. Diversos livros de topo- 
logia geral desenvolvem a teoria de redes, mas especificamente sobre o assunto recomendamos o texto Clark (2016).

Definição 4.1. Um conjunto direcionado $(P, \leq)$ é um conjunto parcialmente ordenado (Definição 2.8) satisfazendo a propriedade adicional:

1. Para todo $p, q \in P$, existe $r \in P$ tal que $p \leq r$ e $q \leq r$.

Definição 4.2. Seja um espaço topológico $X$ e um conjunto direcionado $P$. Uma rede é uma função $f: P \rightarrow X$. Podemos denotar uma rede $f: P \rightarrow X$ como $\left(x_{p}\right)_{p \in P}$, em que $x_{p}=f(p)$. Uma rede $\left(x_{p}\right)_{p \in P}$ sobre $X$ converge para um ponto $x$ se para toda vizinhança $V$ de $x$ existe $q \in P$ tal que para todo $r \geq q, x_{r} \in V$. Como com sequências, denotamos este fato por $x_{p} \rightarrow x$.

É fácil ver que uma sequência nada mais é do que um caso particular de rede, onde $P=\mathbb{N}$ com a ordem usual. Para subredes precisaremos de mais duas definições.

Definição 4.3. Sejam $P$ e $Q$ conjuntos direcionados. Dizemos que uma função $f: Q \rightarrow P$ é monótona se para todo $q_{1}, q_{2} \in Q$, temos que $q_{1} \leq q_{2}$ implica $f\left(q_{1}\right) \leq f\left(q_{2}\right)$.

A função $f$ é cofinal se para todo $p \in P$, existe $q \in Q \operatorname{com} p \leq f(q)$.

Dada uma sequência $\left(x_{n}\right)_{n \in \mathbb{N}}$, uma subsequência $\left(x_{n_{k}}\right)_{k \in \mathbb{N}}$ é definida por uma função $f: \mathbb{N} \rightarrow \mathbb{N}$ estritamente crescente (portanto cofinal em $\mathbb{N}$ ) em que $n_{k}=f(k)$. Uma definição natural para subredes seria: dada uma rede $\left(x_{p}\right)_{p \in P},\left(x_{p_{q}}\right)_{q \in P}$ é uma subrede se existe uma função $f: P \rightarrow P$ monótona e cofinal em que $p_{q}=f(q)$.

Essa definição, porém, é muito restritiva para nosso objetivo, que é generalizar dois resultados válidos para sequências em espaços métricos para espaços topológicos arbitrários (Proposições 4.5 e 4.6).

Neste trabalho utilizaremos a definição de subrede de Willard, que segue mais de perto a definição de subsequência. Uma discussão concisa sobre as diferentes definições de subrede pode ser encontrada nas notas de apresentação de Martin Sleziak, Sleziak (2009).

Definição 4.4. Seja $P$ um conjunto direcionado e $\left(x_{p}\right)_{p \in P}$ uma rede sobre um conjunto $X$. Se $Q$ é um conjunto direcionado e $f: Q \rightarrow P$ é uma função monótona cofinal, então $\left(x_{f(q)}\right)_{q \in Q}$ é uma subrede de $\left(x_{p}\right)_{p \in P \text {. }}$

Uma rede $\left(x_{n}\right)_{n \in \mathbb{N}}$ sobre $\mathbb{N}$ com a ordem usual é, também, uma sequência, mas uma subrede de $\left(x_{n}\right)_{n \in \mathbb{N}}$ não necessariamente é uma subsequência. Essa diferença fica clara na demonstração da proposição a seguir.

Proposição 4.5. Toda rede sobre um espaço topológico compacto admite uma sub-rede convergente. 
Demonstração. Seja $(P, \preccurlyeq)$ um conjunto direcionado e $\left(x_{p}\right)_{p \in P}$ uma rede em um espaço compacto $X$. Para cada $p \in P$, defina o conjunto

$$
F_{p}:=\overline{\left\{x_{q} \in X \mid p \preccurlyeq q\right\}} .
$$

Note que a família $\left\{F_{p}\right\}_{p \in P}$ tem a Propriedade da Interseção Finita, e como $X$ é compacto, existe $x \in \bigcap_{p \in P} F_{p}$. Defina

$$
Q:=\left\{(U, p) \in \mathscr{P}(X) \times P \mid x_{p} \in U \text { e } U \text { é vizinhança de } x\right\}
$$

com a ordem $(U, p) \leq(V, r)$ se, e só se, $V \subset U$ e $p \preccurlyeq r$. É fácil verificar que $(Q, \leq)$ é um conjunto direcionado. Vamos mostrar que $\left(x_{f(q)}\right)_{q \in Q}$ é uma subrede de $\left(x_{p}\right)_{p \in P}$ com a função $f: Q \rightarrow P$ definida por $f((U, p))=p$.

É claro pela definição que $f$ é monótona. Também é simples ver que $f$ é cofinal, pois dado qualquer $p \in P$, temos que $(X, p) \in Q$ para todo $p \in P$. Mostramos que $\left(x_{f(q)}\right)_{q \in Q}$ é subrede de $\left(x_{p}\right)_{p \in P}$, resta mostrar que $x_{f(q)} \rightarrow x$.

Seja $U_{0}$ uma vizinhança de $x$. Note que, por definição, $U_{0}$ intercepta cada um dos conjuntos $F_{p}$, logo, existe $p_{0} \in P$ de modo que $x_{p_{0}} \in U_{0}$. Assim, $\left(U, p_{0}\right) \in Q$.

Para todo $(U, p) \in Q \operatorname{com}\left(U_{0}, p_{0}\right) \preccurlyeq(U, p)$, temos que $x_{f(q)}=x_{p} \in V \subset U$, e temos o resultado. Por fim resta mostrar que $x_{f((U, p))} \rightarrow x$, mas isso é claro pois dada qualquer vizinhança $U$ de $x$, existe $p \in P$ com $x_{p} \in U$, então para todo $(V, r) \geq(U, p)$, temos que $x_{f(V, r)}=x_{r} \in V \subset U$.

Imediatamente, por esta demonstração, concluímos que para todo $A \subset X, x \in \bar{A}$ se, e somente se, existe uma rede $\left(x_{p}\right)_{p \in P}$ em $A$ que converge para $x$. Basta considerar $P=\{U \subset$ $X \mid U$ é vizinhança de $x\}$ com a ordem $U \leq V \Leftrightarrow V \subset U$ e uma função $f: P \rightarrow X \operatorname{com} f(U) \in$ $U \cap A$, então é claro que $x_{p} \rightarrow x$. Note que a função $f$ existe, pois $x \in \bar{A}$, logo, se $U$ é vizinhança de $X, U \cap A \neq \emptyset$, além disso, a existência da função que determina um elemento para cada conjunto é garantida pelo Axioma da Escolha.

Note também que a proposição anterior não é válida para sequências, uma vez que as únicas sequências convergentes em $\beta \mathbb{N}$ são eventualmente constantes (Hindman e Strauss (2012), Corolário 3.42.1).

As redes também nos dão uma caracterização completa da continuidade de funções, o que não ocorre com sequências.

Proposição 4.6. Seja $f: X \rightarrow Y$ uma função. A função $f$ é contínua em $x$ se, e somente se, para toda rede $\left(x_{p}\right)_{p \in P}$ sobre $X$, se $x_{p} \rightarrow x$, então $f\left(x_{p}\right) \rightarrow f(x)$.

Demonstração. Suponha que para toda rede $\left(x_{p}\right)_{p \in P} \operatorname{com} x_{p} \rightarrow x$ tenhamos que $f\left(x_{p}\right) \rightarrow f(x)$. Suponha que $f$ não seja contínua em $x$, então existe uma vizinhança $V$ de $f(x)$ de modo que $f^{-1}(V)$ não é vizinhança de $x$. Considere $P=\{U \subset X \mid U$ é vizinhança de $x\}$ com a ordem da inclusão reversa, então $P$ é um conjunto direcionado.

Para cada $p \in P$, defina um ponto $x_{p} \in U \backslash f^{-1}(V)$. Note que este ponto sempre existe pois 
$f^{-1}(V)$ não é vizinhança de $x$, e assim $f\left(x_{p}\right) \notin V$.

É claro que $x_{p} \rightarrow x$, porém para todo $p \in P, f\left(x_{p}\right) \notin V$, então $f\left(x_{p}\right) \nrightarrow f(x)$, contradizendo nossa hipótese.

A demonstração da recíproca é idêntica à demonstração deste fato para sequências.

Como contra-exemplo para este fato envolvendo sequências, considere sobre $\mathbb{R}$ as topologias $\sigma$ dos conjuntos coenumeráveis e $\tau$ a topologia usual. Note que a função $f:(\mathbb{R}, \sigma) \rightarrow(\mathbb{R}, \tau)$ dada por $f(x)=x$ não é contínua, pois $(0,1) \in \sigma$, mas $f^{-1}[(0,1)]=(0,1)$ não é um aberto de $\tau$. $\operatorname{Em}(\mathbb{R}, \sigma)$, um sequência $\left(x_{n}\right)_{n \in \mathbb{N}}$ é convergente se, e somente se, é eventualmente constante, ou seja, existe $N \in \mathbb{N}$ tal que $x_{n}=x_{m} \forall n, m \geq N$. Assim, é claro que, se $x_{n} \rightarrow x$ em $(\mathbb{R}, \sigma)$, então $f\left(x_{n}\right)=x_{n} \rightarrow x$ em $(\mathbb{R}, \tau)$.

Intuitivamente podemos pensar que um ponto é limite de uma sequência se a quantidade de elementos da sequência que estão próximos a esse ponto é grande. A maneira como estenderemos o conceito de limite é modificando o que "grande" significa.

Seja $X$ um espaço topológico e $\left(x_{n}\right)_{n \in \mathbb{N}}$ uma sequência de $X$. Um ponto $x \in X$ é o limite da sequência se

$$
\text { para toda vizinhança } V \text { de } x, \exists N \in \mathbb{N}, x_{n} \in V \forall n \geq N \text {. }
$$

Ou equivalentemente:

para toda vizinhança $V$ de $x,\left\{n \in \mathbb{N} \mid x_{n} \in V\right\}$ tem complementar finito.

Dessa forma fica explícito quais conjuntos são grandes em $\mathbb{N}$ por essa definição. Comentamos no primeiro capítulo que os ultrafiltros nos dão uma forma de definir conjuntos grandes de $\mathbb{N}$. De fato, cada ultrafiltro define uma medida (finitamente aditiva) em $\mu: \mathscr{P}(X) \rightarrow\{0,1\}$, e isso nos leva à seguinte definição: seja $p$ um ultrafiltro sobre $\mathbb{N}$, dizemos que $p-\lim _{n} x_{n}=x$ se, e somente se,

$$
\text { para toda vizinhança } V \text { de } x,\left\{n \in \mathbb{N} \mid x_{n} \in V\right\} \in p .
$$

Considere um sistema dinâmico $(X, T)$ sobre $\mathbb{N}$. Qualquer $x \in X$ determina uma sequência $\left(T^{n}(x)\right)_{n \in \mathbb{N}}$, ou seja, uma função $f: \mathbb{N} \rightarrow X$ dada por $f(n)=T^{n}(x)$. Como $X$ é compacto de Hausdorff, podemos estender continuamente tal função a $\tilde{f}: \beta \mathbb{N} \rightarrow X$.

Considere um ultrafiltro $p \in \beta \mathbb{N}$ e tome $y=\tilde{f}(p)$. Pela maneira como definimos a extensão $\tilde{f}$ (última parte do Teorema 2.26), vemos que para toda vizinhança $U$ de $y,\{n \in \mathbb{N} \mid f(n) \in U\} \in p$, portanto $\left\{n \in \mathbb{N} \mid T^{n}(x) \in U\right\} \in p$, o que coincide com a definição acima.

Definição 4.7. Seja $\left(X,\left\langle T_{S}\right\rangle_{s \in S}\right)$ um sistema dinâmico. Para cada $x \in X$ e $p \in \beta S$ definimos $T_{p}(x)=p$ - $\lim _{s} T_{S}(x)$ como $\tilde{f}(p)$ onde $\tilde{f}$ é a única extensão contínua da função $f: S \rightarrow X$ dada por $f(s)=T_{S}(x)$. Para um conjunto $\left(x_{s}\right)_{s \in S} \subset X$ qualquer, definimos analogamente o elemento $p$ - $\lim _{s} x_{s}$ para cada $p \in \beta S$.

Proposição 4.8. Seja um sistema dinâmico $\left(X,\left\langle T_{S}\right\rangle_{s \in S}\right)$. Para todo $x \in X$, temos $\left\{T_{p}(x) \mid p \in\right.$ $\beta S\}=\overline{\left\{T_{S}(x) \mid s \in S\right\}}$. 
Demonstração. Fixe $x \in X$ e seja $p \in \beta S$. Se $y=T_{p}(x)$ então para toda vizinhança $V$ de $y$ temos $\left\{s \in S \mid T_{S}(x) \in V\right\} \in p, \operatorname{logo} y \in \overline{\left\{T_{S}(x) \mid s \in S\right\}}$. Mostramos então que $\left\{T_{p}(x) \mid p \in \beta S\right\} \subset$ $\overline{\left\{T_{S}(x) \mid s \in S\right\}}$.

Por outro lado, seja $y \in \overline{\left\{T_{S}(x) \mid s \in S\right\}}$. Para cada vizinhança $V$ de $y$, defina $N_{V}=\left\{s \in S \mid T_{S}(x) \in\right.$ $V\}$. Note que a família de conjuntos $\left\{N_{V} \mid V\right.$ vizinhança de $\left.y\right\}$ tem a propriedade da interseção finita, portanto pode ser estendida a um ultrafiltro $p$, portanto $T_{p}(x)=y$.

Note que esta generalização nos permitiu caracterizar o fecho de um conjunto, que intuitivamente são os pontos próximos dele. Veremos que a noção de recorrência em um sistema dinâmico está intimamente relacionada com os ultrafiltros por esse motivo. Além disso, como acontece com limites usuais, funções contínuas preservam limites.

Lema 4.9. Seja $X$ um espaço compacto de Hausdorff e $\left(x_{s}\right)_{s \in S} \subset X$. Para qualquer função $f$ : $X \rightarrow Y$ contínua onde $Y$ é um compacto de Hausdorff, temos que $f\left(p-\lim _{s} x_{s}\right)=p$ - $\lim _{s}\left(f\left(x_{s}\right)\right)$.

Demonstração. Seja $g: S \rightarrow X$ a função definida por $g(s)=x_{s}$ e tome $\tilde{g}: \beta S \rightarrow X$ sua única extensão contínua, assim, $f\left(p-\lim _{s} x_{s}\right)=f \circ \tilde{g}(p)$, e note que $f \circ \tilde{g}$ é uma função contínua. Por outro lado, temos a função $f \circ g: S \rightarrow Y$ que pode ser estendida continuamente a $(f \tilde{\circ} g)$, e $p-\lim _{s} f\left(x_{s}\right)=(f \circ g)(p)$.

Porém $(f \tilde{\circ} g)$ e $f \circ \tilde{g}$ são funções contínuas que coincidem em $S$, logo, são iguais.

Para $x \in X$ fixo, a função $\tilde{f}: \beta S \rightarrow X$ dada por $\tilde{f}(p)=T_{\mathscr{U}}(x)$ é contínua, pois é extensão contínua de $f: S \rightarrow X$, em que $f(s)=T_{s}(x)$. Agora se fixarmos $p \in \beta S$, podemos definir a função $g: X \rightarrow X$ dada por $g(x)=T^{p}(x)$, mas nesse caso temos garantida a continuidade de $g$ apenas se $p$ for um ultrafiltro principal.

Já discutimos como estender a operação do semigrupo para os ultrafiltros sobre ele, e agora mostraremos que essa operação é plenamente compatível com a estrutura do sistema dinâmico.

Proposição 4.10. Seja $\left(X,\left\langle T_{S}\right\rangle_{s \in S}\right)$ um sistema dinâmico. Para todo $p, q \in \beta S, T_{p} \circ T_{q}=T_{p+q}$.

Demonstração. Seja $V$ uma vizinhança de $T_{p+q}(x)$.

$$
\begin{aligned}
T_{p+q}(x) \in V & \Leftrightarrow\left\{t \in S \mid T_{t}(x) \in V\right\} \in p+q \\
& \Leftrightarrow\left\{s \in S \mid-s+\left\{t \in S \mid T_{t}(x) \in V\right\} \in q\right\} \in p \\
& \Leftrightarrow\left\{s \in S \mid\left\{t \in S \mid T_{s+t}(x) \in V\right\} \in q\right\} \in p \\
& \Leftrightarrow\left\{s \in S \mid T_{S}\left(T_{q}(x)\right) \in V\right\} \in p \\
& \Leftrightarrow T_{p}\left(T_{q}(x)\right) \in V
\end{aligned}
$$

Para ver que vale a penúltima equivalência, note que

$$
\left\{s \in S \mid\left\{t \in S \mid T_{s+t}(x) \in V\right\} \in q\right\}=\left\{s \in S \mid q-\lim _{t}\left(T_{s+t}(x)\right) \in V\right\}
$$


mas $s \in S$, então $T_{s}$ é contínua. Pelo resultado anterior, $q-\lim _{t}(T s+t(x))=T_{s}\left(q-\lim _{t}\left(T_{t}(x)\right)\right.$, portanto

$$
\left\{s \in S \mid q-\lim _{t}\left(T_{s+t}(x)\right) \in V\right\}=\left\{s \in S \mid T_{s} \circ T_{q}(x) \in V\right\}
$$

A seguir examinaremos alguns conceitos de recorrência em sistemas dinâmicos. Para um sistema dinâmico usual $(X, T)$, em que o semigrupo em questão é $(\mathbb{N},+)$, dizemos que um ponto $x \in X$ é recorrente se para toda vizinhança $V$ de $x$, o conjunto $\left\{n \in \mathbb{N} \mid T^{n}(x) \in V\right\}$ é infinito, mas além disso estamos interessados se este conjunto possui alguma regularidade, e para isso definimos os conjuntos sindéticos.

Nos naturais, dizemos que um $A \subset \mathbb{N}$ é sindético se possui gaps limitados, ou seja, se existe $M \in \mathbb{N}$ tal que para todo $n \geq M$, temos

$$
A \cap\{n+1, n+2, \ldots, n+M\} \neq \emptyset .
$$

Note que implicitamente estamos usando a ordem usualmente associada aos números naturais, o que não pode ser estendido a semigrupos arbitrários. Temos, portanto, a seguinte definição equivalente:

Definição 4.11. Seja $S$ um semigrupo. Um conjunto $A \subset S$ é sindético se existe $G \subset S$ finito tal que $S=\bigcup_{g \in G}-g+A$.

Nos naturais, se existe $M \in \mathbb{N}$ com $A \cap\{n+1, n+2, \ldots, n+M\} \neq \emptyset$ para todo $n \in \mathbb{N}$, então é fácil ver que $\mathbb{N}=\bigcup_{i=1}^{M+1}-i+A$. Reciprocamente, suponha $\mathbb{N}=\bigcup_{g \in G}-g+A \operatorname{com} G \subset \mathbb{N}$ finito e defina $M=\max (G)+1$. Afirmamos que $A \cap\{n+1, \ldots, n+M\} \neq \emptyset$ para todo $n \in \mathbb{N}$. De fato, se supormos o contrário, ou seja, que existe $n \in \mathbb{N}$ tal que $A \cap\{n+1, \ldots, n+M\}=\emptyset$, então $n+1>a-g$ para todo $g \in G$ e $a \in A$ com $a<n+1$ e, por outro lado, se $a>n+1$, então $a \geq n+M=n+\max (G)+1, \log 0 n+1<a-g$ para todo $g \in G$, o que contradiz a hipótese inicial de que $\mathbb{N}=\bigcup_{g \in G}-g+A$. Note que se o semigrupo não for comutativo, deveríamos distinguir os casos $S=\bigcup_{t \in F}-t+A$ e $S=\bigcup_{t \in F} A-t$, mas esta distinção não será útil no decorrer deste trabalho.

Definição 4.12. Seja $\left(X,\left\langle T_{S}\right\rangle_{s \in S}\right)$ um sistema dinâmico. Então:

- $x \in X$ é recorrente se existe uma rede $\left(s_{p}\right)_{p \in P}$ sobre $S$ tal que $T_{s_{p}}(x) \rightarrow x$.

- $x \in X$ é uniformemente recorrente se para toda vizinhança $V$ de $x,\left\{s \in S \mid T_{S}(x) \in V\right\}$ é sindético.

- $x, y \in X$ são proximais se existe uma rede $\left(s_{p}\right)_{p \in P}$ sobre $S$ tal que as redes $\left(T_{s_{p}}(x)\right)_{p \in P}$ e $\left(T_{s_{p}}(y)\right)_{p \in P}$ convergem para o mesmo ponto. 
Equivalentemente, um ponto $x \in X$ é recorrente se para toda vizinhança $U$ de $x$, o conjunto $\left\{s \in S \mid T_{S}(x) \in U\right\}$ é infinito.

Para o caso em que $S=\mathbb{N}$, um ponto $x$ é uniformemente recorrente se para toda vizinhança $U$ de $x$, existe $M \in \mathbb{N}$ de modo que se $T^{n}(x) \in U$, então $T^{n+m}(x) \in U$ para algum $m \leq M$.

Para o caso em que $X$ é métrico e $S=\mathbb{N}$, os pontos $x$ e $y$ são proximais se existe alguma sequência $\left(n_{k}\right)_{k \in \mathbb{N}}$ de naturais tal que $d\left(T^{n_{k}}(x), T^{n_{k}}(y)\right) \rightarrow 0$, podendo ocorrer inclusive de as sequências $\left(T^{n_{k}}(x)\right)_{k \in \mathbb{N}}$ e $\left(T^{n_{k}}(y)\right)_{n \in \mathbb{N}}$ não convergirem.

O próximo teorema explicita como os ultrafiltros captam a proximidade entre um ponto e uma rede (ou sequência).

Teorema 4.13. Seja $\left(X,\left\langle T_{S}\right\rangle_{s \in S}\right)$ um sistema dinâmico.

1. $x \in X$ é recorrente $\Leftrightarrow T^{p}(x)=x$ para algum $p \in \beta S$ não principal $\Leftrightarrow T^{p}(x)=x$ para algum $p \in \beta S$.

2. $x \in X$ é uniformemente recorrente $\Leftrightarrow$ para todo $q \in \beta S$, existe $p \in \beta S \operatorname{com} T_{p}\left(T_{q}(x)\right)=x$.

3. $x, y \in X$ são proximais $\Leftrightarrow T_{p}(x)=T_{p}(y)$ para algum $p \in \beta S$.

Demonstração. 1) Seja $x$ recorrente. Seja $\left(s_{p}\right)_{p \in P}$ tal que $T_{s_{p}}(x) \rightarrow x$. Para cada vizinhança $V$ de $x$, defina $N_{V}=\left\{s_{p} \mid T_{s_{p}}(x) \in V\right\}$ e defina $\mathscr{N}=\left\{N_{V} \subset S \mid V\right.$ é vizinhança de $\left.x\right\}$. Note que $\mathscr{N}$ tem a propriedade da interseção finita, portanto podemos estendê-lo a um ultrafiltro $p$ e teremos $T_{p}(x)=x$. Se $p=s \in S$, então $T_{n s}(x)=x \forall n \in \mathbb{N}$ ( $n s$ denota $s+s+\cdots+s n$ vezes), então o ultrafiltro $q$ contendo $\{n s \mid n \in \mathbb{N}\}$ e os conjuntos cofinitos não é principal e $T_{q}(x)=x$.

Reciprocamente, suponha $T_{p}(x)=x$. Seja $\left(s_{i}\right)_{i \in I}$ uma rede sobre $S$ convergindo para $p$ (existe pois $\bar{S}=\beta S$ ). Note que $T_{p}(x)=\lim _{p} T_{s_{p}}(x)=x$.

2) Suponha $x$ uniformemente recorrente. Seja $q \in \beta S$. Fixe uma vizinhança fechada $V$ de $x$, então $\left\{s \in S \mid T_{S}(x) \in V\right\}$ é sindético e seja $F \subset S$ como na definição de sindeticidade. Defina os conjuntos $P_{j}=-j+\left\{s \in S \mid T_{s}(x) \in V\right\}=\left\{s \in S \mid T_{j+s}(x) \in V\right\}$ para cada $j \in F$. Como $q$ é ultrafiltro e $\bigcup_{j \in F} P_{j}=S$, existe $j_{0} \in F$ com $P_{j_{0}}=\left\{s \in S \mid T_{j_{0}+s}(x) \in V\right\} \in q$, portanto $T_{j_{0}}\left(T_{q}(x)\right) \in V$, pois $V$ é fechado (precisamos da hipótese de $V$ ser fechado pois $T_{q}$ é definido por um limite).

Como $X$ é compacto de Hausdorff, admite sistema de vizinhanças fechadas, provamos então que para qualquer vizinhança $V$ (não necessariamente fechada), o conjunto $Y_{V}=\left\{s \in S \mid T_{s}\left(T_{q}(x)\right) \in\right.$ $V\}$ é não vazio.

Note que $Y_{V_{1}} \cap Y_{V_{2}}=Y_{V_{1} \cap V_{2}}$, assim, o conjunto de todos os $Y_{V}$ tem a P.I.F., logo, gera um filtro que pode ser estendido a um ultrafiltro $p$, e assim, $\left\{s \in S \mid\left\{r \in S \mid T_{s+r}(x) \in V\right\} \in q\right\} \in p$, ou seja, $T_{p}\left(T_{q}(x)\right) \in V$ para toda vizinhança $V$.

Reciprocamente, se $x$ não é uniformemente recorrente, existe uma vizinhança aberta $V$ de $x$ de modo que para, todo $F \subset S$ finito, o conjunto

$$
Y_{F}=\left\{s \in S \mid(\forall j \in F) T_{j+s}(x) \notin V\right\}
$$


é não vazio. Note que, para dois conjuntos finitos $F_{1}, F_{2} \subset S, Y_{F_{1}} \cap Y_{F_{2}}=Y_{F_{1} \cup F_{2}}$. Assim, o conjunto $\left\{Y_{F} \subset S \mid F \subset S\right.$ é finito $\}$ tem a PIF. Defina um ultrafiltro $q \in \beta S$ tal que $Y_{F} \in q$ para todo $F \subset S$ finito.

Para todo $r \in S$, como $Y_{\{r\}} \in q$, temos

$$
\left\{s \in S \mid T_{r+s}(x) \notin V\right\}=\left\{s \in S \mid T_{S}(x) \notin T_{r}^{-1}(V)\right\}=\left\{s \in S \mid T_{S}(x) \in X \backslash T_{r}^{-1}(V)\right\} \in q .
$$

Novamente, como $X \backslash T_{r}^{-1}(V)$ é fechado, temos que $T_{q}(x) \in X \backslash T_{r}^{-1}(V)$ para todo $r \in S$. Assim, para todo $p \in \beta S, T_{p}\left(T_{q}(x)\right) \notin V$, então $T_{p}\left(T_{q}(x)\right) \neq x$.

3) Suponha $x$ e $y$ proximais, então existe uma rede $\left(s_{p}\right)_{p \in P}$ de modo que as redes $\left(T_{s_{p}}(x)\right)_{p \in P} \mathrm{e}$ $\left(T_{s_{p}}(y)\right)_{p \in P}$ convergem para um mesmo ponto. Possivelmente passando para uma sub-rede, temos que $s_{p} \rightarrow p$ para algum $p \in \beta S$, e neste caso temos que $T_{p}(x)=\lim _{p \in P} T_{s_{p}}(x)=\lim _{p \in P} T_{s_{p}}(y)=$ $T_{p}(y)$.

Suponha $T_{p}(x)=T_{p}(y)$. Seja $\left(s_{p}\right)_{p \in P}$ uma rede sobre $S$ que converge a $p$, então $T_{p}(x)=$ $\lim _{p \in P} T_{s_{p}}(x)=\lim _{p \in P} T_{s_{p}}(y)=T_{p}(y)$.

\subsection{Universalidade de $\beta S$}

A propriedade de qualquer função contínua de $S$ para um espaço compacto de Hausdorff poder ser estendida continuamente a $\beta S$ garante a universalidade do sistema dinâmico $\left(\beta S,\left\langle\lambda_{s}\right\rangle_{s \in S}\right)$. Intuitivamente isso nos permite reproduzir a dinâmica de um espaço $X$ arbitrário em $\beta S$.

Como aplicação deste conceito conseguimos o Teorema de Auslander-Ellis, que garante que qualquer ponto de um sistema dinâmico é proximal a algum elemento uniformemente recorrente.

Definição 4.14. Seja $S$ um semigrupo. Dizemos que $\left(X,\left\langle T_{S}\right\rangle_{s \in S}\right)$ é um sistema dinâmico universal sobre $S$ se para todo sistema dinâmico $\left(Y,\left\langle R_{S}\right\rangle_{s \in S}\right)$ existe uma função contínua $\varphi: X \rightarrow Y$ de modo que para todo $s \in S$, temos que $R_{S} \circ \varphi=\varphi \circ T_{S}$, ou seja, o seguinte diagrama é comutativo.

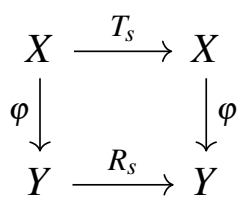

Teorema 4.15. O sistema dinâmico $\left(\beta S,\left\langle\lambda_{S}\right\rangle_{s \in S}\right)$ é universal sobre $S$.

Demonstração. Seja $\left(X,\left\langle T_{S}\right\rangle_{s \in S}\right)$ um sistema dinâmico sobre $S$ e fixe $x \in X$. Considere a função $f: S \rightarrow X$ como $f(s)=T_{S}(x)$, e defina $\varphi: \beta S \rightarrow X$ como a única extensão contínua de $f$. Sejam $r, s \in S$, temos

$$
T_{r} \circ \varphi(s)=T_{r}\left(T_{s}(x)\right)=\varphi(r+s)=\varphi \circ \lambda_{r}(s)
$$


Do mesmo modo que fizemos para $\left(X,\left\langle T_{S}\right\rangle_{s \in S}\right)$, para todo $p \in S^{*}$ podemos definir os limites generalizados da família de funções $\left\langle\lambda_{s}\right\rangle_{s \in S}$ como $\lambda_{p}(q)=p-\lim _{s} \lambda_{s}(q)$. Seja $A \subset \mathbb{N}$ com $p+q \in \widehat{A}$, então pela definição de soma, temos

$$
A \in \lambda_{p}(q) \Leftrightarrow\left\{s \in S \mid \lambda_{s}(q) \in \widehat{A}\right\} \in p \Leftrightarrow\{s \in S \mid s+q \in \widehat{A}\} \in p \Leftrightarrow A \in p+q .
$$

Assim, $\lambda_{p}(q)=p+q$, como era esperado. Podemos então caracterizar pontos recorrentes e proximais a partir da operação de semigrupo estendida a $\beta S$.

Denotaremos por 0 a identidade do semigrupo $S$.

Teorema 4.16. Considere o sistema dinâmico $\left(\beta S,\left\langle\lambda_{s}\right\rangle_{s \in S}\right)$, então:

1. $p$ é recorrente em $\beta S \Leftrightarrow q+p=$ p para algum $q \neq 0$.

2. $p$ é uniformemente recorrente em $\beta S \Leftrightarrow$ para cada $q \in \beta S$, existe $r \in \beta S$ com $r+q+p=p$ $\Leftrightarrow$ p pertence a um ideal à esquerda minimal (fechado) de $\beta S$.

3. $p_{1}$ e $p_{2}$ são proximais em $\beta S \Leftrightarrow$ existe $q$ tal que $q+p_{1}=q+p_{2}$.

4. $p$ gera um subsemigrupo minimal fechado de $\beta S \Leftrightarrow p$ é idempotente $(p+p=p)$.

Demonstração. (1) e (3) são consequências imediatas do Teorema 4.13 aplicado a este sistema dinâmico específico, assim como a primeira equivalência em (2). Vamos mostrar então a segunda equivalência.

Primeiro suponha que $p$ pertence a um ideal minimal à esquerda $I$ e seja $q \in \beta S$, então $I=$ $\beta S+p=\beta S+(q+p)$ (pois $p+\beta S$ é minimal, $q+p \in \beta S+p$ e aplicando Proposição 3.6), portanto temos $p \in \beta S+(q+p)$, ou seja, existe algum $r \in \beta S \operatorname{com} r+q+p=p$.

Reciprocamente, suponha que para cada $q \in \beta S$ exista $r \in \beta S$ de modo que $r+q+p=p$ e que $p$ não pertença a um ideal à esquerda minimal, ou seja, $\beta S+p$ não seja ideal à esquerda minimal. Seja $q^{\prime} \in \beta S+p$ de modo que $\beta S+q^{\prime} \subsetneq \beta S+p$ (note que $\beta S+q^{\prime}$ é ideal à esquerda). Como $q^{\prime}=q+p$ para algum $q \in \beta S$, temos que $\beta S+q+p \subsetneq \beta S+p$. Por hipótese existe $r \in \beta S$ tal que $r+q+p=p$, portanto $p \in \beta S+q+p$ o que implica $\beta S+p \subset \beta S+q+p$, uma contradição. (4) Se $p$ é idempotente, então $\{p\}$ é subsemigrupo minimal fechado. Vamos mostrar a recíproca. Seja $F$ o subsemigrupo minimal fechado gerado por $p$. Note que $F+p$ é subsemigrupo fechado de $F$, pois $F$ é compacto e $F+p$ é imagem de $F$ pela função $\rho_{p}$. Por minimalidade temos que $F+p=F$, então o conjunto $A=\{q \in F \mid q+p=p\}$ é não vazio, fechado (pré-imagem contínua de $\{p\})$ e é subsemigrupo de $F$, já que

$$
q, r \in A \Rightarrow(q+r)+p=q+(r+p)=q+p=p .
$$

Portanto $p \in A \Rightarrow p+p=p$.

Com o teorema anterior e a estrutura algébrica de $\beta S$ conseguimos um primeiro resultado de recorrência. 
Corolário 4.17. Seja $(S,+)$ um semigrupo fracamente cancelativo à esquerda (Definição 3.20), então existe $p \in S^{*}$ uniformemente recorrente em $\left(\beta S,\left\langle\lambda_{s}\right\rangle_{s \in S}\right)$.

Demonstração. Pela Proposição 3.22 e Corolário 3.18, existe um ideal à esquerda minimal contido em $S^{*}$, e pelo item (2) do teorema anterior, qualquer um de seus elementos é uniformemente recorrente.

Para generalizarmos o resultado anterior para sistemas dinâmicos arbitrários precisamos utilizar a universalidade de $\beta S$, que relaciona o comportamento de ultrafiltros em $\left(\beta S,\left\langle\lambda_{s}\right\rangle_{s \in S}\right)$ com o de pontos em um sistema dinâmico qualquer.

Teorema 4.18. Seja $\left(X,\left\langle T_{S}\right\rangle_{s \in S}\right)$ um sistema dinâmico e seja $x \in X$, então:

1. Se pé recorrente em $\beta S$, então $T_{p}(x)$ é recorrente em $X$.

2. Se pé uniformemente recorrente em $\beta S$, então $T_{p}(x)$ é uniformemente recorrente em $X$.

3. Se $p_{1}$ e $p_{2}$ são proximais em $\beta S$, então $T_{p_{1}}(x)$ e $T_{p_{2}}(x)$ são proximais em $X$.

Demonstração. (1) Se $p$ é recorrente, então existe $q \in \beta S$ tal que $q+p=p$, portanto $T_{q}\left(T_{p}(x)\right)=$ $T_{q+p}(x)=T_{p}(x)$.

(2) Se $p$ é uniformemente recorrente, para todo $q \in \beta S$, existe $r \in \beta S$ com $r+q+p=p$, e assim, $T_{r}\left(T_{q}\left(T_{p}(x)\right)\right)=T_{r+q+p}(x)=T_{p}(x)$.

(3) Suponha $p_{1}$ e $p_{2}$ proximais. Existe $q \in \beta S$ de modo que $q+p_{1}=q+p_{2}$, então $T_{q}\left(T_{p_{1}}(x)\right)=$ $T_{q}\left(T_{p_{2}}(x)\right)$.

Teorema 4.19. Seja $\left(\beta S,\left\langle\lambda_{s}\right\rangle_{s \in S}\right)$ um sistema dinâmico e suponha que o semigrupo $(S,+)$ possua uma identidade 0 . Se p é uniformemente recorrente e proximal a 0 , então p é idempotente; e se p é idempotente, então pé recorrente e proximal a 0.

Demonstração. Primeira parte: se $p$ é proximal a 0 , então existe $q \operatorname{com} q+p=q+0=q$. Para tal $q$, existe $r \operatorname{com} r+q+p=p$. Combinando as equações,

$$
r+q+p=r+q=p \Rightarrow r+q+p=p+p=p .
$$

Segunda parte: Tome $q=p$ na definição de recorrência e proximalidade.

O último teorema desta seção foi demonstrado originalmente usando um elemento uniformemente recorrente e proximal a 0 . Uma possibilidade para generalizá-lo seria adicionar artificialmente uma unidade ao semigrupo $S$ para que o conceito de proximalidade de 0 tenha sentido, porém podemos utilizar um conceito equivalente, o de ultrafiltro idempotente minimal. 
Proposição 4.20. Seja o sistema dinâmico $\left(\beta S,\left\langle\lambda_{s}\right\rangle_{s \in S}\right)$ e suponha que o semigrupo $(S,+)$ possua uma identidade 0 . Um ultrafiltro $p$ é uniformemente recorrente e proximal a 0 se, e somente se, $p$ é idempotente e pertence a algum ideal à esquerda minimal (dizemos que $p$ é um idempotente minimal).

Demonstração. Pelo item (2) do Teorema 4.16, $p$ é uniformemente recorrente se, e somente se, pertence a algum ideal à esquerda minimal. Além disso, temos

$$
p \text { é idempotente } \Leftrightarrow p+p=p=p+0 \Leftrightarrow p \text { e } 0 \text { são proximais. }
$$

E temos o resultado.

Por fim, temos o principal resultado da seção.

Teorema 4.21 (Auslander-Ellis). Seja $\left(X,\left\langle T_{S}\right\rangle_{s \in S}\right)$ um sistema dinâmico no qual $S$ é um semigrupo fracamente cancelativo à esquerda. Para cada $x \in X$, existe $y \in X$ uniformemente recorrente e proximal a $x$.

Demonstração. Como $S$ é fracamente cancelativo à esquerda, temos que $S^{*}$ é ideal à esquerda, portanto contém um ideal à esquerda minimal que por sua vez contém um elemento $p$ idempotente. Assim temos que $p$ é uniformemente recorrente pelo Teorema 4.16 e consequentemente $T_{p}(x)$ é uniformemente recorrente pelo Teorema 4.18 .

Como $p$ é idempotente, temos que

$$
T_{p}\left(T_{p}(x)\right)=T_{p+p}(x)=T_{p}(x) .
$$

Portanto pelo Teorema 4.13, $x$ e $T_{p}(x)$ são proximais.

\subsection{Sistemas Dinâmicos Minimais}

Como o nome sugere, um sistema dinâmico é minimal se ele não contém propriamente nenhum outro sistema dinâmico. Intuitivamente estes são os sistemas mais simples possíveis, pois podem estar contidos em uma estrutura maior, mas não podem ser decompostos. Mostraremos que $\left(\beta S,\left\langle\lambda_{s}\right\rangle_{s \in S}\right)$ não só é universal como contém sistemas dinâmicos minimais universais.

Definição 4.22. Seja $\left(X,\left\langle T_{S}\right\rangle_{s \in S}\right)$ um sistema dinâmico. Um conjunto $Y \subset X$ é invariante se para todo $s \in S$ temos que $T_{S}(Y) \subset Y$.

Proposição 4.23. Considere o sistema dinâmico $\left(\beta S,\left\langle\lambda_{s}\right\rangle_{s \in S}\right)$. Um conjunto $A \subset \beta S$ é invariante fechado se, e somente se, é um ideal à esquerda fechado de $\beta S$. 
Demonstração. Seja $A \subset \beta S$ um conjunto invariante fechado. Seja $p \in A$, então para todo $s \in S$, $\lambda_{s}(p)=s+p \in A$, portanto $S+p \subset A$. Mas $A$ é fechado, então $\overline{S+p}=\beta S+p \subset A$ (Proposição 3.16).

Seja $L \subset \beta S$ um ideal à esquerda fechado. Para todo $s \in S$ e todo $p \in L, \lambda_{s}(p)=s+p \in L$, portanto $L$ é invariante.

Exemplo 4.24. Um sistema dinâmico é minimal se dentro dele não existem "subsistemas". Formalmente, um sistema dinâmico é minimal se não possui subconjuntos invariantes fechados próprios. Considere $X=[0,1]$ e $T: X \rightarrow X$ dada por $T(x)=\frac{x}{2}$. O conjunto $\left\{\frac{1}{2^{n}} \mid n \in \mathbb{N}\right\} \cup\{0\}$ é fechado invariante e próprio, portanto esse sistema não é minimal. Neste caso é fácil verificar o Teorema de Auslander-Ellis, pois 0 é ponto fixo e para todo $x \in[0,1], d\left(T^{n}(x), 0\right) \rightarrow 0$, ou seja, 0 é uniformemente recorrente e proximal a todo $x \in X$.

Seja agora $X=[0,1]$, mas modificamos $T$ para $T(x)=\overline{\pi x}^{1}$, a multiplicação módulo 1 . Neste caso não é tão claro o comportamento do sistema, mas pode-se mostrar que a órbita de qualquer ponto $x \in[0,1]$, ou seja, o conjunto $\mathscr{O}(x):=\left\{T^{n}(x) \mid n \in \mathbb{N}\right\}$, é densa, o que caracteriza um sistema dinâmico minimal. Equivalentemente, para todo $x \in[0,1],\left\{T^{p}(x) \mid p \in \beta \mathbb{N}\right\}=X$, pela Proposição 4.8.

Lema 4.25. Um sistema dinâmico $\left(X,\left\langle T_{S}\right\rangle_{s \in S}\right)$ é minimal se, e somente se, para todo $x \in X$, $\overline{\mathscr{O}(x)}=X$.

Demonstração. Suponha $\left(X,\left\langle T_{S}\right\rangle_{s \in S}\right)$ minimal e seja $y \in \overline{\mathscr{O}(x)}$. Note que existe uma rede $\left(x_{n}\right)_{p \in P} \subset \mathscr{O}(x) \operatorname{com} x_{n} \rightarrow y$, o que, pela definição de $\mathscr{O}(x)$, nos dá uma rede $\left(s_{p}\right)_{p \in P} \subset S$ tal que $T_{S_{n}}(x) \rightarrow y$. Fixe $s \in S$, como $T_{s}$ é contínua, temos que $T_{S}\left(T_{S_{n}}(x)\right) \rightarrow T_{S}(y) \operatorname{com} T_{S}\left(T_{S_{n}}(x)\right) \in \mathscr{O}(x)$ para todo $p \in P$. Mostramos então que $\overline{\mathscr{O}(x)}$ é invariante, $\log _{\mathrm{o}} \overline{\mathscr{O}(x)}=X$ por minimalidade.

Reciprocamente, seja $A \subset X$ um conjunto invariante fechado. Note que para todo $x \in A$, temos que $\mathscr{O}(x) \subset A$, e por $A$ ser fechado temos que $\overline{\mathscr{O}(x)}=X \subset A$.

Corolário 4.26. Um conjunto $A \subset \beta S$ é invariante fechado minimal (com relação à inclusão) se, e somente se, é um ideal à esquerda minimal.

Demonstração. Segue do lema anterior e do fato que ideais à esquerda minimais são fechados (Corolário 3.18).

Definição 4.27. Seja $S$ um semigrupo. Dizemos que $\left(X,\left\langle T_{S}\right\rangle_{s \in S}\right)$ é um sistema dinâmico universal minimal sobre $S$ se é um sistema dinâmico minimal e para todo sistema dinâmico minimal $\left(Y,\left\langle R_{S}\right\rangle_{s \in S}\right)$ existe uma função contínua sobrejetiva $\varphi: X \rightarrow Y$ de modo que para todo $s \in S$, temos que $R_{S} \circ \varphi=\varphi \circ T_{S}$, ou seja, o seguinte diagrama é comutativo.

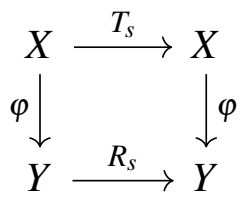


Teorema 4.28. Seja $S$ um semigrupo, L um ideal à esquerda minimal de $\beta S$ e para cada $s \in S$, $\lambda_{s}^{\prime}=\lambda_{s}\left\lceil_{L}\right.$. Então $\left(L,\left\langle\lambda_{s}^{\prime}\right\rangle_{s \in S}\right)$ é um sistema dinâmico minimal universal.

Demonstração. Seja $\left(X,\left\langle T_{S}\right\rangle_{s \in S}\right)$ um sistema dinâmico minimal. Fixe $x \in X$ e considere a função $f: S \rightarrow X$ definida por $f(s)=T_{s}(x)$. Se $\tilde{f}: \beta S \rightarrow X$ é a única extensão contínua de $f$, então definimos $\varphi=\tilde{f} \uparrow_{L}$. Note que pela demonstração do Teorema 4.15 temos que para todo $s \in S$, $\tilde{f} \circ \lambda_{s}=T_{s} \circ \tilde{f}$, portanto $\varphi \circ \lambda_{s}^{\prime}=T_{s} \circ \varphi$.

A sobrejetividade de $\varphi$ é consequência de $X$ ser minimal, $\log _{\mathrm{o}} \overline{\mathscr{O}(x)}=X$ e de que $\left\{T_{p}(x) \in\right.$ $X \mid p \in \beta S\}=\overline{\left\{T_{S}(x) \in X \mid s \in S\right\}}$.

Lema 4.29. Seja $S$ um semigrupo e considere $L, L^{\prime} \subset \beta S$ ideais à esquerda minimais. Se $\varphi$ : $L \rightarrow L^{\prime}$ é um função contínua tal que $\lambda_{s} \circ \varphi=\varphi \circ \lambda_{s}^{\prime}$ para todo $s \in S$, então existe $p \in L^{\prime}$ tal que $\varphi=\rho_{p} \Upsilon_{L}$.

Demonstração. Seja $q \in L$ um ultrafiltro idempotente e considere $p=\varphi(q)$. As funções $\rho_{p} \mathrm{e}$ $\varphi \circ \rho_{q}$ são contínuas de $\beta S$ em $L^{\prime}$. Além disso, dado $s \in S$,

$$
\rho_{p}(s)=s+p=s \varphi(q)=\lambda_{s} \circ \varphi(q)=\varphi \circ \lambda_{s}^{\prime}(q)=\varphi(s+q)=\varphi \circ \rho_{q}(s) .
$$

Como $\rho_{p}=\varphi \circ \rho_{q}(s)$ em $S$ e tais funções admitem uma única extensão contínua, temos que elas coincidem em $\beta S$. Mas como $q$ é uma identidade à direita de $L$, temos que $\varphi \circ \rho_{q} \uparrow_{L}=\varphi$, portanto $\varphi=\rho_{p} \Upsilon_{L}$.

Teorema 4.30. Seja $S$ um semigrupo e $L \subset \beta S$ um ideal à esquerda minimal. $S e\left(X,\left\langle T_{S}\right\rangle_{s \in S}\right) e^{\prime}$ um sistema dinâmico universal minimal sobre $S$, então existe um homeomorfismo $\varphi: L \rightarrow X$ tal que $\varphi \circ \lambda_{s}=T_{s} \circ \varphi$ para todo $s \in S$.

Demonstração. Considere as funções $\varphi: L \rightarrow X$ e $\tau: X \rightarrow L$ garantidas pela minimalidade de ambos os sistemas dinâmicos. Temos o seguinte diagrama.

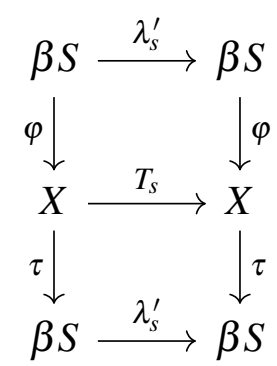

Como $\varphi$ é uma função contínua entre dois compactos, sabemos que $\varphi$ é uma função fechada, portanto para mostrar que ela é, de fato, um homeomorfismo basta demonstrar sua injetividade. Como o diagrama é comutativo, temos que $\tau \circ \varphi: L \rightarrow L$ satisfaz $\lambda_{s}^{\prime} \circ(\tau \circ \varphi)=(\tau \circ \varphi) \circ$ $\lambda_{s}^{\prime}$, portanto pelo lema anterior sabemos que existe $p \in L$ tal que $\tau \circ \varphi=\rho_{p} \uparrow_{L}$, que é um homeomorfismo como visto na demonstração da Proposição 3.24. 



\section{5}

\section{TEORIA DE RAMSEY}

Um dos princípios básicos da Teoria de Ramsey é que não importa quão caoticamente particionarmos um objeto matemático, alguma das partes permanecerá organizada. Usualmente, e em grande parte deste trabalho, lidamos com os números naturais. Como exemplo temos Teorema de Schur, um dos resultados mais antigos da área.

Teorema 5.1 (Schur). Seja $r \in \mathbb{N}$ e $\mathbb{N}=\bigcup_{j=1}^{r} A_{j}$. Existem $i \in\{1, \ldots, r\}$ e $m, n \in \mathbb{N}$ tais que $m \neq n e\{m, n, m+n\} \subset A_{i}$.

Podemos pensar que temos um objeto $X$ e que $\mathscr{G} \subset \mathscr{P}(X)$ são os subconjuntos "organizados" de $X$. No caso do enunciado anterior teríamos $X=\mathbb{N}$ e $\mathscr{G}=\{\{m, n, m+n\} \mid m, n \in$ $\mathbb{N}, m \neq n\}$.

Dizemos que $\mathscr{G}$ é fracamente regular por partições se para qualquer partição finita $A_{1}, \ldots, A_{r}$ de $X$, existem $j \in\{1, \ldots, r\}$ e $G \in \mathscr{G}$ de modo que $G \subset A_{j}$, portanto o teorema anterior pode ser reformulado da seguinte maneira:

Teorema 5.2 (Schur). O conjunto $\mathscr{G}=\{\{m, n, m+n\} \mid m, n \in \mathbb{N}, m \neq n\}$ é fracamente regular por partições em $\mathbb{N}$.

Escolhemos esta definição por ser mais presente nas referências utilizadas neste trabalho, mas usualmente estes resultados são enunciados em termos de colorações.

Imagine que tenhamos uma paleta com $r \in \mathbb{N}$ cores e vamos colorir cada elemento de $\mathbb{N}$ com uma dessas cores. Podemos então definir a função $c: \mathbb{N} \rightarrow\{1, \ldots, r\}$ em que $c(n)=i$ se, e somente se, o número $n$ foi colorido com a $i$-ésima cor da nossa paleta. Note que isso define uma partição finita de $\mathbb{N}$, na qual cada cor determina uma das partes, ou seja, $\mathbb{N}=\bigcup_{i=1}^{r} f^{-1}(i)$. Assim, um conjunto $A \subset \mathbb{N}$ é monocromático, ou seja, todos os seus elementos foram coloridos por uma mesma cor, se e somente se, está contido em uma das partes $f^{-1}(i)$.

$\mathrm{O}$ enunciado seguinte é, portanto, equivalente aos dois anteriores. 
Teorema 5.3 (Schur). Para toda coloração finita de $\mathbb{N}$, existem dois números $m, n \in \mathbb{N}$ distintos de modo que o conjunto $\{m, n, m+n\}$ é monocromático.

Utilizando o item (4) da Proposição 2.7, podemos facilmente reformular estes enunciados para o contexto de ultrafiltros.

Teorema 5.4. Seja $X$ um conjunto e $\mathscr{G} \subset \mathscr{P}(X)$. São equivalentes:

1. G é fracamente regular por partições.

2. Existe um ultrafiltro $p$ sobre $X$ tal que para todo $A \in p$, existe $G \in \mathscr{G}$ tal que $G \subset A$.

Demonstração. $1 \Rightarrow 2$ ) Suponha que $\emptyset \notin \mathscr{G}$ (se $\emptyset \in \mathscr{G}$ então qualquer ultrafiltro satisfaz a condição), e defina

$$
\mathscr{A}=\{B \subset X \mid B \cap G \neq \emptyset \forall G \in \mathscr{G}\} .
$$

Note que $\mathscr{A}$ tem a propriedade da interseção finita, pois se supormos que existam $A_{1}, \ldots, A_{n} \in \mathscr{A}$ $\operatorname{com} \bigcap_{i=1}^{n} A_{i}=\emptyset$, então $\bigcup_{i=1}^{n} X \backslash A_{i}=X$ é uma partição de $X$, mas nenhum $G \in \mathscr{G}$ está contido em nenhum desses conjuntos, contrariando a hipótese de $\mathscr{G}$ ser fracamente regular por partições. Podemos então estender $\mathscr{A}$ a um ultrafiltro $p$, então dado qualquer $A \in p, X \backslash A \notin \mathscr{A}$, portanto existe $G \in \mathscr{G}$ tal que $G \cap(X \backslash A)=\emptyset$, $\log$, $G \subset A$.

$2 \Rightarrow 1)$ Seja $X=\bigcup_{i=1}^{r} A_{i}$ uma partição finita e $p \in \beta S$ como no enunciado. Existe $j \in\{1, \ldots, r\}$ $\operatorname{com} A_{j} \in p$, então existe $G \in \mathscr{G} \operatorname{com} G \subset A_{j}$.

A demonstração da maioria dos resultados deste capítulo segue um mesmo roteiro: é dada uma propriedade $P$ e queremos mostrar que a família

$$
\mathscr{G}_{P}:=\{G \subset S \mid G \text { satisfaz a propriedade } P\}
$$

é fracamente regular por partições. Se existir um conjunto não vazio

$$
\begin{aligned}
\mathfrak{U}_{P} & :=\left\{p \in \beta S \mid \forall A \in p, \exists G \in \mathscr{G}_{P} \text { com } G \subset A\right\} \\
& =\{p \in \beta S \mid \text { todo elemento de } p \text { contém um conjunto que satisfaz a propriedade } P\}
\end{aligned}
$$

poderemos utilizar o resultado anterior para concluir a demonstração. Assim, nosso plano será utilizar as propriedades algébricas de $\beta S$ para determinar a existência de $\mathfrak{U}_{P}$.

\subsection{Teorema de Ramsey}

Este é, provavelmente, o resultado mais conhecido da Teoria de Ramsey, especialmente em sua versão finita na Teoria de Grafos.

Este teorema tem uma diferença fundamental quando comparado com os demais que serão apresentados neste trabalho: ele é um resultado sobre conjuntos, e não sobre semigrupos. Assim, 
os resultados envolvendo semigrupos são considerados uma subárea, chamada combinatória aditiva, devido ao papel da operação, usualmente denotada pela soma, do semigrupo.

Definição 5.5. Sejam $X$ um conjunto e $k \in \mathbb{N}$. Denotamos por $[X]^{k}$ o conjunto de todos os subconjuntos de $X$ com exatamente $k$ elementos.

Perceba a diferença entre os conjuntos $X^{k} \mathrm{e}[X]^{k}$. O primeiro tem como elementos uma sequência com $k$ entradas, portanto no caso $k=2$ e tomando dois elementos distintos $x, y \in X$, temos que $(x, y) \neq(y, x)$ e $(x, x)$ é um elemento de $X^{2}$. Em $[X]^{2}$, os elementos são conjuntos, $\operatorname{logo}\{x, x\}=\{x\}$ não é um elemento de $[X]^{2}$, pois tem apenas um elemento, e $\{x, y\}=\{y, x\}$, pois ambos denotam o conjunto contendo apenas $x$ e $y$.

Lema 5.6. Sejam $X$ um conjunto, $p$ um ultrafiltro não principal sobre $X$ e $r, k \in \mathbb{N}$. Suponha dada uma partição finita $[X]^{k}=\bigcup_{i=1}^{r}$.

Para cada $\alpha \in[X]^{k-1}$ (portanto $\alpha=\left\{x_{1}, \ldots, x_{k-1}\right\}$ ), defina

$$
B_{k}(\alpha, i)=\left\{y \in X \mid \alpha \cup\{y\} \in A_{i}\right\}
$$

Agora, indutivamente, para todo $t \in\{1, \ldots, k-1\}$, todo $\alpha \in[X]^{t-1}$ e todo $i \in\{1, \ldots, r\}$, definimos

$$
B_{t}(\alpha, i)=\left\{y \in X \mid B_{t+1}(\alpha \cup\{y\}, i) \in p\right\} .
$$

Para todo $t \in\{1, \ldots, k\}$ e todo $\alpha \in[X]^{t-1}, S \backslash E=\bigcup_{i=1}^{r} B_{t}(\alpha, i)$.

Demonstração. Para o caso $t=k$, dado $\alpha \in[X]^{k-1}$, então para todo $y \in X \backslash \alpha$, temos que $\alpha \cup\{y\} \in[X]^{k}$, portanto existe $j \in\{1, \ldots, r\}$ com $\alpha \cup\{y\} \in A_{j}$, ou seja, $y \in B_{k}(\alpha, j)$.

Considere $t \in\{1, \ldots, k-1\}$ e, por indução, suponha que o resultado seja válido para todo $\ell \in\{t+1, \ldots, k\}$.

Seja $\alpha \in[X]^{t-1}$ e considere $y \in X \backslash \alpha$. Pela hipótese de indução, $X \backslash(\alpha \cup\{y\})=\bigcup_{i=1}^{r} B_{t+1}(\alpha \cup$ $\{y\}, i)$. Note que $\alpha \cup\{y\}$ é finito, portanto $\alpha \cup\{y\} \notin p$, logo existe algum $j \in\{1, \ldots, r\}$ tal que $B_{t+1}(\alpha \cup\{y\}, j) \in p$, ou seja, $y \in B_{t}(\alpha, j)$.

Para a demonstração do Teorema de Ramsey para o caso $[X]^{2}$, dada uma partição finita $[X]^{2}=\bigcup_{i=1}^{r} A_{i}$, fixamos um ultrafiltro não principal $p$ sobre $X$ e construímos uma função $g: X \rightarrow\{1, \ldots, r\}$ definida como

$$
g(x)=j \Leftrightarrow\left\{y \in X \mid\{x, y\} \in A_{j}\right\} \in p .
$$

O lema anterior nos permite uma generalização dessa ideia, para que possamos demonstrar o caso geral.

Teorema 5.7 (Ramsey). Sejam $r, k \in \mathbb{N}$ e X um conjunto infinito. Se $[X]^{k}=\bigcup_{i=1}^{r} A_{i}$, então existem $j \in\{1, \ldots, r\}$ e um conjunto $C \subset X$ infinito tal que $[C]^{k} \subset A_{j}$. 
Demonstração. Fixe um ultrafiltro não principal $p$ sobre $X$.

Para o caso $k=1$, o enunciado apenas afirma que existe algum conjunto infinito contido em alguma das partes. Note que existe $j \in\{1, \ldots, r\} \operatorname{com} A_{j} \in p$, e como $p$ é não principal, $C=A_{j} \subset A_{j}$ é infinito.

Seja $k \geq 2$ e suponha, por indução, que o resultado seja válido para todo $n<k$.

Definimos os conjuntos $B_{t}(\alpha, i)$ da mesma forma que fizemos no lema anterior. Note que $X=\bigcup_{i=1}^{r} B_{1}(\emptyset, i)$, portanto existe $j \in\{1, \ldots, r\}$ tal que $B_{1}(\emptyset, j) \in p$.

Tome $x_{1} \in B_{1}(\emptyset, j)$, então, pela definição, $B_{2}\left(\left\{x_{1}\right\}, j\right) \in p$.

Por indução, seja $n \in \mathbb{N}$ e suponha que já tenhamos escolhido um conjunto $\left\{x_{1}, \ldots, x_{n}\right\}$ de modo que, para todo $t \in\{1, \ldots, k-1\}$ e todo $m_{1}<m_{2}<\cdots<m_{t} \leq n$, tenhamos

$$
B_{t+1}\left(\left\{x_{m_{1}}, \ldots, x_{m_{t}}\right\}, j\right) \in p
$$

Tome um elemento $x_{n+1}$ distinto dos anteriores tal que

$$
x_{n+1} \in \bigcap\left\{B_{t+1}\left(\left\{x_{m_{1}}, \ldots, x_{m_{t}}\right\}, j\right) \mid t \in\{0,1, \ldots, k-1\}, m_{1}<m_{2}<\cdots<m_{t} \leq n\right\}
$$

Note que tal elemento existe, pois o conjunto acima é a interseção de finitos conjuntos do ultrafiltro $p$.

Precisamos mostrar que $x_{n+1}$ satisfaz a propriedades exigida pela hipótese de indução, ou seja,que $B_{t+1}\left(\left\{x_{m_{1}}, \ldots, x_{m_{t}}\right\}, j\right) \in p$ para todo $t \in\{1, \ldots, k-1\}$ e todo $m_{1}<m_{2}<\cdots<m_{t} \leq n+1$.

Seja $m_{1}<m_{2}<\cdots<m_{t} \leq n+1$ e $t \in\{1, \ldots, k-1\}$. Se $m_{t} \leq n$ estamos ainda no caso da hipótese de indução, portanto $B_{t+1}\left(\left\{x_{m_{1}}, \ldots, x_{m_{t}}\right\}, j\right) \in p$.

Suponha então que $m_{t}=n+1$. Para o caso $t=1, B_{2}\left(\left\{x_{n+1}\right\}, j\right) \in p$, pois $x_{n+1} \in B_{1}(\emptyset, j)$. Para $t>1, B_{t+1}\left(\left\{x_{m_{1}}, \ldots, x_{m_{t}}\right\}, j\right) \in p$, pois $x_{n+1} \in B_{t}\left(\left\{x_{m_{1}}, \ldots, x_{m_{t-1}}\right\}, j\right)$.

Construímos, assim, uma sequência infinita $\left(x_{n}\right)_{n \in \mathbb{N}}$ e definimos $C=\left\{x_{n} \mid n \in \mathbb{N}\right\}$.

Um elemento de $[C]^{k}$ é um conjunto $\left\{x_{m_{1}}, \ldots, x_{m_{k}}\right\}$ com $m_{1}<m_{2}<\ldots, m_{k}$. Pela forma como escolhemos esses elementos, temos que $x_{m_{k}} \in B_{k}\left(\left\{x_{m_{1}}, \ldots, x_{m_{k-1}}\right\}, j\right)$, portanto $\left\{x_{m_{1}}, \ldots, x_{m_{k}}\right\} \in$ $A_{j}$ ), ou seja, $[C]^{k} \subset A_{j}$.

\subsection{Teorema de Van Der Waerden}

O Teorema de Van der Waerden é um dos resultados mais clássicos da Teoria de Ramsey, tendo sido demonstrado em 1927, antes mesmo do Teorema de Ramsey.

O enunciado original trata especificamente do semigrupo $(\mathbb{N},+)$, mas aqui já vemos uma clara vantagem na abordagem que utilizamos com a compactificação de Stone-Čech de semigrupos. Argumentos puramente combinatórios normalmente utilizam propriedades específicas dos naturais, fazendo com que não seja claro como generalizá-los para outros casos. No nosso caso, ao contrário, temos uma teoria tratando de semigrupos arbitrários, o que nos permite recorrer a argumentos combinatórios em pontos específicos, onde é fácil verificar se é possível ou não a generalização. 
Para $k \in \mathbb{N}$ e $s \in S$, utilizamos $k s$ para denotar $s+s+\cdots+s$ iterado $k$ vezes.

Teorema 5.8 (Van der Waerden). Seja $r \in \mathbb{N} e \bigcup_{i=i}^{r} A_{i}=S$ uma partição finita do semigrupo $S$. Existe $j \in\{1, \ldots, r\}$ tal que, para cada $k \in \mathbb{N}$, existem $a, d \in S \operatorname{com}\{a, a+d, \ldots, a+k d\} \subset A_{j}$.

neste caso estamos procurando um conjunto da partição que contenha progressões aritméticas arbitrariamente longas, o que motiva a seguinte definição:

Definição 5.9. Seja $(S,+)$ um semigrupo. Um conjunto $A \subset S$ é dito AP-rich se para todo $k \in \mathbb{N}$, existem $a \in S$ e $d \in S$ tais que $\{a, a+d, \ldots, a+k d\} \subset A$.

Assim, o Teorema de Van der Waerden diz que a família dos conjuntos AP-rich é fracamente regular por partições.

Para a demonstração utilizaremos também o conceito de conjunto sindético por partes, fortemente relacionado aos conjuntos sindéticos (Definição 4.11).

Como para conjuntos sindéticos, a definição de conjuntos sindéticos por partes nos naturais admite uma formulação equivalente: um conjunto $A \subset \mathbb{N}$ é sindético por partes se existem $M \in \mathbb{N}$ e intervalos arbitrariamente longos de $\mathbb{N}$ de modo que o comprimento dos gaps de $A$ sejam limitados por $M$, ou seja, dentro desses infinitos intervalos, qualquer intervalo de comprimento $M$ intersepta $A$. É claro que um conjunto sindético é, também, sindético por partes.

Novamente perceba que implicitamente estamos utilizando a ordem dos naturais, por isso precisamos de uma definição mais geral para semigrupos arbitrários.

Definição 5.10. Seja $(S,+)$ um semigrupo. Um conjunto $A \subset S$ é sindético por partes se existe $G \subset S$ finito tal que $\left\{s^{-1}\left(\bigcup_{g \in G} g^{-1} A\right) \mid s \in S\right\}$ tem a propriedade da interseção finita.

O plano para a demonstração do Teorema 5.8 é bastante simples: primeiro mostraremos que existem ultrafiltros cujos elementos são todos sindéticos por partes, o que implica a família de conjuntos sindéticos por partes ser fracamente regular por partições pelo Teorema 5.4; a seguir mostraremos que conjuntos sindéticos por partes são AP-rich, o que nos dará o resultado.

Antes de passar para a demonstração, ressaltamos que o teorema não garante que existam $a, d \in S$ com $\{a+k d \mid k \in \mathbb{N}\}$ contido em algum dos elementos da partição, ou seja, não necessariamente contém uma progressão infinta. Considere $(\mathbb{N},+)$, vamos particioná-lo em dois conjuntos $A$ e $B$. Começamos com $1 \in A$, e os próximos dois números, 2 e 3 , definimos que pertencem a $B$. Os três números seguintes pertencem a $A$ e os próximos quatro a $B$ e assim sucessivamente, como abaixo

$$
\begin{array}{cccccccccccccccccccccc}
1 & 2 & 3 & 4 & 5 & 6 & 7 & 8 & 9 & 10 & 11 & 12 & 13 & 14 & 15 & 16 & 17 & 18 & 19 & 20 & 21 & 22 \\
A & B & B & A & A & A & B & B & B & B & A & A & A & A & A & B & B & B & B & B & B & A
\end{array}
$$

E assim por diante. Fixe $a, d \in \mathbb{N}$ quaisquer. Na partição que definimos existem dois intervalos consecutivos de comprimento $d$ e $d+1$ contidos em conjuntos distintos e $\{a+n d \mid n \in \mathbb{N}\}$ conterá elementos de ambos os intervalos, portanto elementos tanto de $A$ quanto de $B$. 
Teorema 5.11. Seja $S$ um semigrupo e $p \in \beta S$. São equivalentes:

1. $p \in K(\beta S)$. (Definição 3.2)

2. Para todo $A \in p,\{x \in S \mid-x+A \in p\}$ é sindético.

3. Para todo $q \in \beta S, p \in \beta S+q+p$.

Demonstração. $1 \Rightarrow 2$ ) Seja $A \in p$ e $B=\{x \in S \mid-x+A \in p\}$. Pelo Teorema 3.13, existe um ideal à esquerda minimal $L$ com $p \in L$. Para todo $q \in L, p \in \beta S+q=\overline{S+q}$, dessa forma existe $s_{q} \in S$ tal que $s_{q}+q \in \widehat{A}$ e $q \in-s_{q}+A$, mas $L$ é compacto 3.18 e $\bigcup_{q \in L} \widehat{-s_{q}+A}$ é uma cobertura aberta de $L$, portanto existe $G \subset S$ finito com $L \subset \bigcup_{g \in G} \widehat{-g+A}$.

Seja $s \in S$, então $s+p \in L$ e existe $g \in G$ tal que $s+p \in \widehat{-g+A} \Rightarrow-g+A \in s+p$, portanto $-(g+s)+A=-s+(-g+A) \in p$. Mas então $g+s \in B \Rightarrow s \in-g+B$. Mostramos então que $S=\bigcup_{g \in G}-g+B$, ou seja, $B$ é sindético.

$2 \Rightarrow 3$ ) Seja $p$ como no enunciado. Suponha que exista $q \in \beta S$ tal que $p \notin \beta S+q+p$, então existe $A \in p$ com $\widehat{A} \cap \beta S+q+p=\emptyset$. Seja $B=\{x \in S \mid-x+A \in p\}$ e tome $G \subset S$ finito com $S=\bigcup_{g \in G}-g+B$, então existe $g \in G$ com $-g+B \in q$. Note que $B \in g+q$, ou seja, $\{x \in S \mid-x+A \in p\} \in g+q$, portanto $A \in g+p+q$, contradição.

$3 \Rightarrow 1)$ Tome $q \in K(\beta S)$, então $p \in \beta S+q+p$, mas $\beta S+q \subset K(\beta S)$ e portanto $\beta S+q+p \subset$ $K(\beta S)+p \subset K(\beta S)$.

Teorema 5.12. Seja $S$ um semigrupo e $A \subset S$. Então $\widehat{A} \cap K(\beta S) \neq \emptyset$ se, e só se, A é sindético por partes.

Demonstração. Primeiro suponha $\widehat{A} \cap K(\beta S) \neq \emptyset$. Seja $p \in K(\beta S) \operatorname{com} A \in p$ e defina $B=\{x \in$ $S \mid-x+A \in p\}$. Pelo teorema anterior sabemos que $B$ é sindético, ou seja, $S=\bigcup_{g \in G}-g+B$ para algum $G \subset S$ finito. Para cada $s \in S$, existe $g_{s} \in G$ tal que $s \in-g_{s}+B$, então pela definição de $B$, $-s+\left(-g_{s}+A\right) \in p, \operatorname{logo},-s+\left(\bigcup_{g \in G}-g+A\right) \in p$ para todo $s \in S$. Como todos os conjuntos dessa forma pertencem a $p$, eles possuem a propriedade da interseção finita.

Reciprocamente, seja $G \subset S$ finito tal que $\left\{-s+\left(\bigcup_{g \in G}-g+A\right) \mid s \in S\right\}$ tenha a propriedade da interseção finita, então existe $p \in \beta S$ tal que $\left\{-s+\left(\bigcup_{g \in G}-g+A\right) \mid s \in S\right\} \subset p$. Note que $S+p \subset$ $\bigcup_{g \in G}-g+A$, pois para todo $s \in S$ sabemos que $\bigcup_{g \in G}-g+A \in s+p$, portanto, pela Proposição 3.16, $\beta S+p \subset \bigcup_{g \in G-g}+A$. Pelo Teorema 3.13 podemos escolher $q \in \beta S+p \cap K(\beta S)$, mas então $q \in \widehat{-g+A}$ para algum $g \in G$, e assim $g+q \in \widehat{A} \cap K(\beta S)$.

Com o corolário a seguir concluímos não só que existem ultrafiltros cujos elementos são todos sindéticos por partes, mas caracterizamos todos esses conjuntos a partir de ultrafiltros, especificamente como elementos de $K(\beta S)$.

É curioso como uma propriedade combinatória de conjuntos de um semigrupo pode ser traduzida como uma estrutura algébrica de sua compactificação. Este fenômeno se repetirá na seção 
seguinte, sobre o Teorema de Hindman, e também quando tratarmos de conjuntos de densidade não nula em $\mathbb{N}$.

Corolário 5.13. Seja $S$ um semigrupo e $p \in \beta S$. Então $p \in \overline{K(\beta S)}$ se, e só se, para todo $A \in p$, $A$ é sindético por partes.

Demonstração. Seja $p \in \overline{K(\beta S)}$. Se $A \in p$, então $\widehat{A}$ é uma vizinhança de $p$, logo, existe $q \in$ $K(\beta S) \cap \widehat{A}$. Pelo teorema anterior, $A$ é sindético por partes.

Reciprocamente, suponha $p \in \beta S$ e para todo $A \in p, A$ é sindético por partes. Note que $\mathscr{B}=$ $\{\widehat{A} \subset \beta S \mid A \in p\}$ é uma base local de $p$, mas para todo $\widehat{A} \in \mathscr{B}$ temos pelo teorema anterior que $\widehat{A} \cap K(\beta S) \neq \emptyset$, portanto $p \in \overline{K(\beta S)}$.

Por fim concluiremos a segunda parte de nosso plano, e assim teremos que para todo ultrafiltro $p \in \overline{K(\beta S)}$, se $A \in p$ então $A$ é um conjunto AP-rich.

Teorema 5.14. Considere $(S,+)$. Se $A \subset S$ é sindético por partes então A é um conjunto AP-rich.

Demonstração. Considere $Y=X_{t=0}^{k} \beta S$, com a operação de $\beta S$ coordenada a coordenada, então $Y$ é um semigrupo topológico à direita compacto e $\chi_{t=0}^{k} S \subset \Lambda(Y)$.

Para cada $p \in \beta S$, defina $\Delta p=(p, p, \ldots, p)$. Defina também

$$
\begin{gathered}
I=\{(a, a+d, a+2 d, \ldots, a+k d) \mid a, d \in S\} \mathrm{e} \\
E=I \cup\{\Delta a \mid a \in S\} .
\end{gathered}
$$

É fácil ver que $E$ é subsemigrupo de $\chi_{t=0}^{k} S$ e que $I$ é ideal de $E$. Pela Proposição 3.27 temos que $\bar{E}$ é subsemigrupo de $Y$ e $\bar{I}$ é ideal de $\bar{E}$, os fechos sendo tomados em $Y$. Além disso, temos que $K(Y)=\chi_{t=0}^{k} K(\beta S)$ pela Proposição 3.26. Afirmamos que $\bar{E} \cap K(Y) \neq \emptyset$, o que implica $K(\bar{E})=\bar{E} \cap K(Y)$ pela Proposição 3.25 .

Seja $p \in K(\beta S)$. Considere uma vizinhança $U$ de $\Delta p$ e para cada $t \in\{0, \ldots, k\}$ defina $A_{t} \in p$ de modo que $\mathrm{X}_{t=0}^{k} A_{t} \subset U$. Assim $\bigcap_{t=0}^{k} A_{t} \in p$, logo existe $a \in \bigcap_{t=0}^{k} A_{t}$ e portanto $\Delta a \in U \cap \bar{E}$.

Note que $\bar{E} \cap \mathrm{X}_{t=0}^{k} K(\beta S)=K(\bar{E}) \subset \bar{I}$, e como $A$ é sindético por partes, pelo Corolário 5.13 existe $p \in \widehat{A} \cap K(\beta S)$, logo, $\Delta p \in K(\bar{E}) \subset I$. Seja $U=\chi_{t=0}^{k} \widehat{A}$ e note que $U$ é vizinhança de $\Delta p$. Portanto $U \cap I \neq \emptyset$, ou seja, existem $a, d \in S \operatorname{com}(a, a+d, \ldots, a+k d) \in A$.

Teorema de Van der Waerden. Seja $p \in \overline{K(\beta S)}$. Para todo $A \in p$, temos que $A$ é sindético por partes pelo Corolário 5.13, mas então $A$ é AP-rich pelo teorema anterior.

Concluímos então que todo conjunto pertencente a $p$ é AP-rich, logo, pelo Teorema 5.4, a família dos conjuntos AP-rich é fracamente regular por partições. 


\subsection{Teorema de Hindman}

No começo do capítulo mencionamos o Teorema de Schur, que diz que o conjunto $\{\{x, y, x+y\} \subset \mathbb{N} \mid x \neq y\}$ é fracamente regular por partições, mas isso é o melhor que podemos conseguir? Será que não podemos conseguir mais pares em uma mesma partição cujas somas ainda estão contidas nela, ou mesmo que existam infinitos pares?

Naturalmente nos perguntamos se $\mathscr{G}=\{G \subset \mathbb{N} \mid G$ é fechado pela soma $\}$ é fracamente regular por partições, ou seja, se dados $x, y \in F$ temos que $x+y \in F$, mas facilmente podemos ver que isso é falso. Para cada $n \in \mathbb{N}$, definimos $k_{n}$ como o maior número de modo que $2^{k_{n}}$ divide $n$ (possivelmente 0 ), então considere a partição:

$$
\begin{aligned}
& n \in A_{0} \Leftrightarrow k_{n} \text { é par } \\
& n \in A_{1} \Leftrightarrow k_{n} \text { é ímpar }
\end{aligned}
$$

Assim, dado qualquer $n \in \mathbb{N}, n \in A_{0}$ se, e só se, $n+n \in A_{1}$ e $n \in A_{1}$ se, e só se, $n+n \in A_{0}$, portanto nem $A_{0}$ nem $A_{1}$ contém algum conjunto fechado pela soma.

Note que se um conjunto $G \subset \mathbb{N}$ é fechado pela soma, então existe algum conjunto infinito $H \subset \mathbb{N}$ de modo que para qualquer sequência finita $\left(h_{1}, h_{2}, \ldots, h_{n}\right)$ de elementos de $H$, temos que $\sum_{i=1}^{n} h_{i} \in F$. Vamos enfraquecer esta definição para que não seja permitido a soma de elementos repetidos de $H$.

Considere agora um conjunto $A \subset \mathbb{N}$ de modo que existe $H \subset \mathbb{N}$ tal que para todo conjunto finito $\left\{h_{1}, h_{2}, \ldots, h_{n}\right\}$ de $H, \sum_{i=1}^{n} h_{i} \in A$. Ao substituir sequências finitas por conjuntos finitos, eliminamos o problema da soma repetida de números.

Para um conjunto $H \subset \mathbb{N}$, denotaremos por $F S(H)$ o conjunto $\left\{\sum_{h \in F} h \mid F \subset H\right.$ é finito $\}$, a soma de finitos elementos distintos de $H$. O Teorema de Hindman, portanto, diz que $\mathscr{G}=$ $\{F S(H) \mid H$ é infinito $\}$ é fracamente regular por partições.

O conjunto considerado acima é apropriado para semigrupos comutativos, como $\mathbb{N}$, onde não nos preocupamos com a ordem com que são feitas as operações, porém para semigrupos arbitrários definiremos que um conjunto $A \subset S$ é um IP-set se existe uma sequência infinita $\left(h_{1}, h_{2}, \ldots\right)$ de $S$ com elementos dois a dois distintos, de modo que para qualquer sequência finita estritamente crescente de $\mathbb{N}\left(n_{1}, n_{2}, \ldots, n_{k}\right)$, temos que $h_{n_{1}}+h_{n_{2}}+\ldots h_{n_{k}} \in A$. Para evitar repetição, acordaremos que quando tivermos o conjunto das somas finitas de uma sequência, estamos sempre lidando com a soma em ordem crescente de índices.

Teorema 5.15 (Hindman). Sejam $S$ um semigrupo infinito, $r \in \mathbb{N}$ e $S=\bigcup_{i=1}^{r} A_{i}$ uma partição finita de S. Existe $j \in\{1, \ldots, r\}$ tal que $A_{j}$ é um IP-set.

Apesar de originalmente IP-set denotar Infinite dimensional Paralelepiped, o termo é pertinente também no contexto de ultrafiltros, pois os IP-sets são justamente os conjuntos que pertencem a algum ultrafiltro IdemPotente. 
Vamos mostrar que todos os elementos de um ultrafiltro idempotente são IP-sets. Como já sabemos que $\beta S$ contém algum ultrafiltro idempotente, teremos o resultado.

Definição 5.16. Seja $p \in \beta S$ e $A \subset S$. Definimos $A^{*}(p)=\{s \in A \mid-s+A \in p\}$.

A princípio este conjunto pode ser vazio, mas se $p$ é um ultrafiltro idempotente não principal e $A \in p$, temos

$$
A^{*}(p)=\{s \in A \mid-s+A \in p\}=\{s \in S \mid-s+A \in p\} \cap A .
$$

Mas como $A \in p$ e $p$ é idempotente:

$$
A \in p \Leftrightarrow A \in p+p \Leftrightarrow\{s \in S \mid-s+A \in p\} \in p .
$$

Portanto $A^{*}(p)$ é a interseção de dois conjuntos pertencentes a $p, \operatorname{logo} A^{*}(p) \in p$ e, em particular, é infinto.

Lema 5.17. Seja $(S,+)$ um semigrupo, $p \in S^{*}$ idempotente e $A \in p$, então $-s+A^{*}(p) \in p \forall s \in$ $A^{*}(p)$.

Demonstração. Sejam $a \in A^{*}(p)$ e $B=-a+A$, então $B \in p$. Lembre-se que um conjunto $X$ pertence a $p+p$ se, e somente se, $\{s \in S \mid-s+X \in p\} \in p$ e que $p=p+p$, logo

$$
\begin{gathered}
B \in p \Leftrightarrow B \in p+p \Leftrightarrow\{s \in S \mid-s+B \in p\} \in p \\
\Leftrightarrow\{s \in S \mid-s+B \in p\} \cap B \in p \Leftrightarrow\{b \in B \mid-b+B \in p\} \in p \Leftrightarrow B^{*}(p) \in p .
\end{gathered}
$$

Vamos mostrar que $B^{*}(p) \subset-a+A^{*}(p)$ e assim teremos que $-a+A^{*}(p) \in p$.

Se $s \in B^{*}(p)$, então $a+s \in A^{*}(p) \subset A$ e $-s+B \in p$, logo, $-s+B=-s+(-a+A)=-(a+$ $s)+A \in p$. Como $a+s \in A$ e $-(a+s)+A \in p$, temos que $a+s \in A^{*}(p) \Rightarrow s \in-a+A^{*}(p)$.

Teorema 5.18 (Hindman). Sejam $S$ um semigrupo infinito, $p \in S^{*}$ um ultrafiltro idempotente e $A \in p$, então A é um IP-set.

Demonstração. Seja $h_{1} \in A^{*}(p)$. Por indução, suponha que a sequência $\left(h_{1}, \ldots, h_{n}\right)$ de elementos de $A^{*}(p)$ tal que $F S\left(\left(h_{i}\right)_{i=1}^{n}\right) \subset A^{*}(p)$ já tenha sido escolhida. Defina $E=F S\left(\left(h_{i}\right)_{i=1}^{n}\right)$ e note que $E$ é finito. Além disso, para cada $a \in E$ temos $-a+A^{*}(p) \in p$ (pelo lema anterior) e portanto $\bigcap_{a \in E}-a+A^{*}(p) \in p$. Como $p \in S^{*}$, o conjunto $\bigcap_{a \in E}-a+A^{*}(p)$ é infinito, portanto existe $h_{n+1} \in A^{*}(p) \cap \bigcap_{a \in E}-a+A^{*}(p) \operatorname{com} h_{n+1} \notin\left\{h_{1} \ldots, h_{n}\right\}$. Assim, $h_{n+1} \in A^{*}(p)$, logo para todo $a \in E, a \cdot h_{n+1} \in A^{*}(p)$, portanto $F S\left(\left(h_{i}\right)_{i=1}^{n+1}\right) \subset A^{*}(p) \subset A$.

Da mesma forma que no Teorema de Van der Waerden, conseguimos mostrar que existem ultrafiltros, mais especificamente os ultrafiltros idempotentes, formados somente por IP-sets, então pelo Teorema 5.4 temos como corolário o Teorema de Hindman do início da seção.

Mais do que isso, podemos mostrar que um conjunto $A \subset S$ é um IP-set se, e somente se, existe um 
ultrafiltro $p \in \beta S$ idempotente com $A \in p$, ou seja, conseguimos também a recíproca do teorema anterior, o que nos permite caracterizar os IP-sets via ultrafiltros. Antes disso precisaremos de dois resultados técnicos auxiliares.

Lema 5.19. Sejam $S$ um semigrupo e $\mathscr{A} \subset \mathscr{P}(S)$ com a propriedade da interseção finita. Se para cada $A \in \mathscr{A}$ e todo $x \in A$ existir $B \in \mathscr{A} \operatorname{com} x+B \subset A$ então $\bigcap_{A \in \mathscr{A}} \bar{A}$ é subsemigrupo de $\beta S$.

Demonstração. Seja $T=\bigcap_{A \in \mathscr{A}} \bar{A}$. Note que $\mathscr{A}$ tem a propriedade da interseção finita, portanto $\mathscr{A}$ pode ser estendido a um ultrafiltro $p \in \beta S$, e note que $p \in T$, ou seja, $T \neq \emptyset$.

Sejam $p, q \in T$ e $A \in \mathscr{A}$. Dado $x \in A$, defina $B \in \mathscr{A}$ tal que $x+B \subset A$ (existe por hipótese), então $B \subset-x+A$ e $-x+A \in q$. Temos então que $A \subset\{x \in S \mid-x+A \in q\}$ e, logo, $\{x \in S \mid-x+A \in$ $q\} \in p$, o que é a definição de $A \in p+q$, portanto $p+q \in T$.

Lema 5.20. Seja $S$ um semigrupo e $\left(h_{i}\right)_{n \in \mathbb{N}}$ uma sequência infinita de $S$ com elementos dois a dois distintos, então $\left.\bigcap_{m \in \mathbb{N}} F S \widehat{\left(h_{i}\right)_{i=m}^{\infty}}\right)$ é um subsemigrupo de $\beta S$. Em particular, existe um idempotente $p \in \beta S$ tal que para todo $m \in \mathbb{N}, F S\left(\left(h_{i}\right)_{i=m}^{\infty}\right) \in p$.

Demonstração. Seja $\left.T=\bigcap_{m \in \mathbb{N}} F S \widehat{\left(h_{i}\right)_{i=m}^{\infty}}\right)$ e vamos usar o lema anterior para mostrar que $T$ é subsemigrupo.

É trivial que a família $\left\{F S\left(\left(h_{i}\right)_{i=m}^{\infty}\right) \mid m \in \mathbb{N}\right\}$ tem a Propriedade da Interseção Finita. Seja $m \in \mathbb{N}$ e $s \in F S\left(\left(h_{i}\right)_{i=m}^{\infty}\right)$. Tome $F \subset \mathbb{N}$ finito com $\min (F) \geq m$ e $s=\sum_{n \in F} h_{n}$ (em ordem crescente de índices). Defina $k=\max (F)+1$ e note que $s+F S\left(\left(h_{i}\right)_{i=k}^{\infty}\right) \subset F S\left(\left(h_{i}\right)_{i=m}^{\infty}\right)$.

Estamos portanto nos termos do lema anterior, $\log T$ é subsemigrupo de $S$.

Em particular, existe um ultrafiltro idempotente $p \in T$, pois $T$ é subsemigrupo compacto de Hausdorff (Teorema 3.17).Assim, $\left.p \in F S \widehat{\left(\left(h_{i}\right)_{i=m}^{\infty}\right.}\right)$ para todo $m \in \mathbb{N}$, $\operatorname{logo} F S\left(\left(h_{i}\right)_{i=m}^{\infty}\right) \in p$ para todo $m \in \mathbb{N}$.

Teorema 5.21. Seja $S$ um semigrupo e $A \subset S$. Existe um idempotente $p \in S^{*}$ com $A \in p$ se, e somente se, A é um IP-set.

Demonstração. O Teorema 5.18 diz que se $p \in S^{*}$ é idempotente então para todo $A \in p, A$ é um IP-set.

Por outro lado, se $A$ é um IP-set, então existe $\left(h_{n}\right)_{i \in \mathbb{N}} \operatorname{com} F S\left(\left(h_{n}\right)_{i \in \mathbb{N}}\right) \subset A, \operatorname{logo} A \in p$ pela segunda parte do lema anterior.

\subsection{Teorema de Hindman para soma e produto}

No caso dos números naturais, podemos considerar os semigrupos $(\mathbb{N},+)$ ou $(\mathbb{N}, \cdot)$, portanto todos os resultados da seção anterior são válidos para ambos. Uma pergunta natural seria se podemos generalizar o Teorema de Hindman para ambas as estruturas simultaneamente, 
mais precisamente, se dada uma partição qualquer, existem dois conjuntos infinitos $H_{1}, H_{2} \subset S$ tais que as somas finitas de $H_{1}$ e os produtos finitos de $H_{2}$ estejam em uma mesma parte.

Se conseguirmos um ultrafiltro sobre $\beta \mathbb{N}$ idempotente tanto com relação à soma quanto ao produto, ou seja, $p=p+p=p \cdot p$, o resultado seguiria trivialmente, mas tais ultrafiltros não existem. ${ }^{1}$

Veremos, porém, que os idempotentes das duas operações estão topologicamente próximos, e isso garantirá o resultado.

Lema 5.22. Seja $(S,+)$ um semigrupo e $\mathscr{A} \subset \mathscr{P}(S)$ uma família de subconjuntos com a propriedade da interseção finita. Seja $(T, \cdot)$ um semigrupo topológico compacto à direita e suponha que $\varphi: S \rightarrow T$ satisfaça $\varphi(S) \subset \Lambda(T)$. Suponha que exista $A \in \mathscr{A}$ tal que para todo $x \in A$ exista $B \in \mathscr{A}$ tal que $\varphi(x+y)=\varphi(x) \cdot \varphi(y) \forall y \in B$. Então para todo $p, q \in \bigcap_{A \in \mathscr{A}} \widehat{A}$ temos que $\tilde{\varphi}(p+q)=\tilde{\varphi}(p) \cdot \tilde{\varphi}(q)$. (Lembrando que $\tilde{\varphi}: \beta S \rightarrow T$ é a extensão contínua de $\varphi$ definindo sobre $S$ a topologia discreta)

Demonstração. Fixe $A, B \in \mathscr{A}$ como garantido pelo enunciado e sejam $p, q \in \bigcap_{C \in \mathscr{A}} \widehat{C}$. Note que se $p \in \bigcap_{C \in \mathscr{A}} \widehat{C}$, então $A, B \in p$. Para todo $x \in A$, temos:

$$
\begin{array}{rlr}
\tilde{\varphi}(x+q) & =\tilde{\varphi} \circ \lambda_{x}\left(q-\lim _{y} y\right) & \\
& =q-\lim _{y} \varphi(x+y) & \left(\text { pois } \tilde{\varphi} \circ \lambda_{x} \text { é contínua }\right) \\
& =q-\lim _{y} \varphi(x) \varphi(y) & (\varphi(x+y)=\varphi(x) \varphi(y) \text { em } B \text { e } B \in q) \\
& =\varphi(x) q-\lim _{y} \varphi(y) & (\varphi(x) \in \Lambda(T)) \\
& =\varphi(x) \tilde{\varphi}(q)
\end{array}
$$

Por outro lado, como $A \in p$, temos:

$$
\begin{array}{rlr}
\tilde{\varphi}(p+q) & =\tilde{\varphi} \circ \rho_{q}\left(p-\lim _{x} x\right) & \\
& =p-\lim _{x} \tilde{\varphi}(x+q) & \left(\tilde{\varphi} \circ \rho_{q}\right. \text { é contínua) } \\
& =p-\lim _{x}(\varphi(x) \tilde{\varphi}(q)) & \text { (conta anterior) } \\
& =\left(p-\lim _{x} \varphi(x)\right) \tilde{\varphi}(q) & \\
& =\tilde{\varphi}(p) \tilde{\varphi}(q) & \text { (continuidade de } \left.\rho_{\tilde{\varphi}}(q)\right)
\end{array}
$$

Em particular, usando o resultado anterior para o caso $\mathscr{A}=\{S\}$, se $\varphi(S) \subset \Lambda(T)$ é um homomorfismo, então $\tilde{\varphi}: \beta S \rightarrow T$ é também homomorfismo.

Além disso, mostraremos agora que essa extensão preserva injetividade e sobrejetividade. 
Lema 5.23. Sejam $X$ e $Y$ dois espaços topológicos discretos e $f: X \rightarrow Y$ uma função (contínua). Se $f$ é injetiva, $\tilde{f}: \beta X \rightarrow \beta Y$ é injetiva. Se $f$ for uma função sobrejetiva, então $\tilde{f}$ é sobrejetiva.

Demonstração. Suponha que existam $p, q \in \beta X$ com $p \neq q$ e $\tilde{f}(p)=\tilde{f}(q)=r$. Existe $A \subset X$ tal que $A \in p$ e $A \notin q$. Porém temos:

$$
\tilde{f}(p)=r \Rightarrow f(A) \in r \Rightarrow f^{-1}(f(A))=A \in q \text { (pela injetividade) } \Rightarrow q \in \widehat{A},
$$

contradição.

Para a segunda parte, suponha $f$ sobrejetiva e seja $p \in \beta Y$ qualquer. Note que o conjunto $\left\{f^{-1}(A) \mid A \in p\right\}$ tem a PIF, portanto existe $q \in \beta X$ tal que $\left\{f^{-1}(A) \mid A \in p\right\} \subset q$. Por construção temos que $f^{-1}(A) \in q$ para toda vizinhança $A$ de $p$, portanto $\tilde{f}(q)=p$.

Lema 5.24. $p \in \overline{E(\beta S)}$ se, e somente se, $A$ é um IP-set para todo $A \in p$.

Demonstração. Seja $p \in E(\beta S)$ e considere uma vizinhança básica $\widehat{A}$ de $p$, então $A \subset S$ e $A \in p$. Como $p \in \overline{E(\beta S)}$, existe $q \in \widehat{A}$ idempotente, ou seja, $A \in q$, e pelo Teorema 5.21, existe um conjunto como no enunciado.

Reciprocamente seja $A \in p$, então $\widehat{A}$ é uma vizinhança de $p$. Como $A$ é um IP-set, pelo Teorema 5.21, existe $q \in \beta S$ idempotente com $A \in q$, logo, $q \in \widehat{A}$.

Os resultados seguintes são específicos para o conjuntos dos naturais, pois examinaremos relações entre as estruturas algébricas de $(\beta \mathbb{N},+)$ e $(\beta \mathbb{N}, \cdot)$. Para maior clareza usaremos uma notação diferente da usada no resto do texto.

Definição 5.25. $I P+:=\{p \in \beta \mathbb{N} \mid p+p=p\}$, o conjunto dos idempotentes de $\beta \mathbb{N}$ com a operação de soma.

$I P m+=\{p \in \beta \mathbb{N} \mid p$ é idempotente minimal em $(\beta \mathbb{N},+)\}$.

Analogamente definimos $I P \cdot$ e $I P m \cdot$ para a operação produto.

Nos lemas seguintes estaremos tratando de $\beta \mathbb{N}$ não isoladamente, mas visto dentro de uma estrutura maior. Para um espaço topológico completamente regular $X$ arbitrário, se $Y \subset X$, não necessariamente temos $\beta Y \subset \beta X$. Como exemplo tome $\mathbb{R}$ com a topologia usual, vamos mostrar que $\mathbb{Q} \subset \mathbb{R}$, mas $\beta \mathbb{Q} \nsubseteq \beta \mathbb{R}$.

Suponha que $\beta \mathbb{Q} \subset \beta \mathbb{R}$, então qualquer função contínua $f: \mathbb{Q} \rightarrow \mathbb{Q}$ admitiria uma extensão contínua $\tilde{f}: \beta \mathbb{Q} \rightarrow \beta \mathbb{Q}$. Como estamos supondo $\beta \mathbb{Q} \subset \beta \mathbb{R}$ temos necessariamente que $\beta \mathbb{Q}=$ $\overline{\mathbb{Q}} \subset \beta \mathbb{R}$ (fecho sendo tomado em $\beta \mathbb{R}$ ) e, assim, $\mathbb{R} \subset \overline{\mathbb{Q}}$. Note que a função $f: \mathbb{Q} \rightarrow \mathbb{Q}$ definida por $f(x)=0$ para $x<\pi$ e $f(x)=1$ para $x>\pi$ é contínua, mas ela não pode ser estendida continuamente para $\mathbb{R}$, portanto não pode ser estendida continuamente para $\beta \mathbb{Q}$, uma contradição. Concluímos então que $\beta \mathbb{Q}$ não está contido em $\beta \mathbb{R}$.

No nosso caso, porém, consideramos a topologia discreta, portanto o problema que tivemos no caso anterior não ocorre, já que toda função em um espaço topológico discreto é contínua. 
Considere um semigrupo $(S,+)$ e $B \subset S$ um subsemigrupo de $S$. Note que $B \subset S$, portanto $B \subset \beta S$, logo podemos tomar o conjunto $\bar{B} \subset \beta S$. Como estamos considerando a topologia discreta sobre ambos os conjuntos, uma função $f: B \rightarrow X$ em que $X$ é um espaço compacto de Hausdorff pode ser estendida continuamente para $g: S \rightarrow X$, que por sua vez admite uma única extensão contínua $\tilde{g}: \beta S \rightarrow X$. Como $B$ é denso em $\bar{B}$ e $\tilde{g}$ restrita a $\bar{B}$ é contínua, temos que $\tilde{g} \uparrow_{B}=f$. Provamos então que $\bar{B}=\beta B$. Note que, para as nossas aplicações, este argumento é válido inclusive para $\mathbb{R}$ e $\mathbb{Q}$, pois não estamos considerando a topologia usual desses semigrupos, e sim a topologia discreta.

Então dizer que um ultrafiltro $p$ em $\beta \mathbb{Q}$ está em $\beta \mathbb{N}$, significa que $p \in \overline{\mathbb{N}}$, ou seja, para qualquer $A \subset \mathbb{Q} \operatorname{com} A \in p$, existe $n \in \mathbb{N}$ tal que $n \in A$, portanto $\mathbb{N} \cap A \neq \emptyset \forall A \in p$, concluímos então que $\mathbb{N} \in p$. Além disso, se $\mathbb{N} \in p$, então $\{A \cap \mathbb{N} \mid A \in p\}$ é um ultrafiltro sobre $\mathbb{N}$.

Mesmo vendo um ultrafiltro $p$ de $\beta \mathbb{N}$ como estando estando em $\beta \mathbb{Q}$, ainda assim temos que $\mathbb{N} \in p$, então para qualquer partição finita de $\mathbb{N}$ teremos que alguma das partes pertencerá a $p$.

Lema 5.26. Seja $p \in \beta \mathbb{N}$ idempotente. Para todo $n \in \mathbb{N}, n \mathbb{N} \in p$, em que $n \mathbb{N}$ denota o conjunto de todos os múltiplos de $n$.

Demonstração. Considere o semigrupo finito $\left(\mathbb{Z}_{n},+\right)$ em que $\mathbb{Z}_{n}=\{0,1, \ldots, n-1\}$ com a operação $+: \mathbb{Z}_{n} \times \mathbb{Z}_{n} \rightarrow \mathbb{Z}_{n}$ dada por $z_{1}+z_{2}={\overline{z_{1}+z_{2}}}^{n}$, a operação soma módulo $n$. Como $\mathbb{Z}_{n}$ é finito, temos que $\mathbb{Z}_{n}=\beta \mathbb{Z}_{n}$.

Seja $\gamma:(\mathbb{Z},+) \rightarrow\left(\mathbb{Z}_{n},+\right)$ o homomorfismo dado por $\gamma(x)=\overline{n x}^{n}$, então pela discussão procedendo o Lema 5.22, $\tilde{\gamma}: \beta \mathbb{Z} \rightarrow \mathbb{Z}_{n}$ é um homomorfismo. Portanto $\tilde{\gamma}(p)=\tilde{\gamma}(p)+\tilde{\gamma}(p)$, logo, $\tilde{\gamma}(p)=0$. Temos então $\gamma^{-1}(\{0\})=n \mathbb{Z} \cup\{0\} \in p$, portanto $n \mathbb{Z} \cap \mathbb{N}=n \mathbb{N} \in p$.

Lema 5.27. Sejam $p \in(\beta \mathbb{N},+)$ idempotente e $\alpha>0$ um número racional, então $\alpha p$ é idempotente em $\beta \mathbb{N}$. Se $p$ é idempotente minimal, então $\alpha p$ também é idempotente minimal.

Demonstração. A função $h_{\alpha}: \mathbb{N} \rightarrow \mathbb{Q}$ definida por $h_{\alpha}(n)=\alpha n$ é um homomorfismo (tanto com relação à soma como à multiplicação) injetivo, portanto sua extensão contínua $\tilde{h_{\alpha}}: \beta \mathbb{N} \rightarrow \beta \mathbb{Q}$ também é um homomorfismo injetivo. Por ser homomorfismo temos que $\alpha p$ é idempotente em $(\mathbb{Q},+)$.

Seja $\alpha=\frac{m}{n}, n, m \in \mathbb{N}$. Pelo lema anterior, sabemos que $n \mathbb{N} \in p$, assim, $\alpha n \mathbb{N}=m \mathbb{N} \in p$, portanto $\mathbb{N} \in \alpha p$, o que implica $\alpha p \in \beta \mathbb{N}$.

Suponha agora que $p$ seja minimal sobre $\mathbb{N}$ e seja $q \leq \alpha p$ um idempotente de $\beta \mathbb{N}$. Note que $\tilde{h_{\alpha^{-1}}}: \beta \mathbb{N} \rightarrow \mathbb{Q}$ é homomorfismo, portanto $\alpha^{-1} q$ é idempotente em $\beta \mathbb{N}$, e é a inversa de $\tilde{h}_{\alpha^{-1}}$. Além disso,

$$
\alpha^{-1} q p=\alpha^{-1} q \alpha^{-1} \alpha p=\tilde{h_{\alpha^{-1}}}(q) \tilde{h_{\alpha^{-1}}}(\alpha p)=\tilde{h_{\alpha^{-1}}}(q \alpha p)=\tilde{h_{\alpha^{-1}}}(q)=\alpha^{-1} q .
$$

Portanto $\alpha^{-1} q \leq p$, mas como $p$ é minimal, $\alpha^{-1} q=p$ e portanto $q=\alpha p$, ou seja, $\alpha p$ é minimal. 
Teorema 5.28. $\overline{I P+} e \overline{I P m+}$ são ideais à esquerda de $(\beta \mathbb{N}, \cdot)$.

Demonstração. Seja $p \in \overline{I P+}$. Vamos mostrar que $n p \in \overline{I P+} \forall n \in \mathbb{N}$.

Seja $n \in \mathbb{N}$ e $A \in n p$. Então $n^{-1} A \in p$ e existe $q \in \beta \mathbb{N}$ idempotente tal que $n^{-1} A \in q$, portanto $A \in$ $n q$. Mas pelo lema anterior $n q$ é idempotente, portanto dada uma vizinhança de $n p$ encontramos um idempotente $n q$ que pertence à ela.

A demonstração da segunda parte é idêntica, porém utilizando a segunda parte do lema anterior.

Corolário 5.29. $(I P m \cdot) \cap \overline{(I P m+)} \neq \emptyset$. Em particular, existe $p \in \overline{I P+}$ tal que $p \cdot p=p$.

Demonstração. Pelo teorema anterior $I P m+$ é um ideal à esquerda de $(\beta \mathbb{N}, \cdot)$, portanto contém um idempotente minimal $p \in I P$. pelo Corolário 3.18.

Corolário 5.30 (Hindman generalizado). Seja $r \in \mathbb{N}$ e $\mathbb{N}=\bigcup_{n=1}^{r} A_{r}$. Existe $i \in\{1, \ldots, r\}$ e dois conjuntos infinitos $H_{1}, H_{2} \subset \mathbb{N}$ tais que $F S\left(H_{1}\right) \subset A_{i}$ e $F P\left(H_{2}\right) \subset A_{i}$.

Demonstração. Pelo corolário anterior existe $p \in \overline{I P+} \operatorname{com} p \cdot p=p$, além disso, existe $i \in$ $\{1, \ldots, r\} \operatorname{com} A_{i} \in p$. Pelo Teorema 5.21 temos o conjunto infinito $H_{2} \subset \mathbb{N}$ e pelo Lema 5.24 temos o conjunto $H_{1} \subset \mathbb{N}$.

\subsection{Generalização dos Teoremas de Hindman e Van der Waerden}

Como mencionado no início do capítulo, as estruturas algébricas geradas por diferentes propriedades fracamente regulares por partições podem ter interseção não vazia, o que nos permite imediatamente generalizar os resultados todos em um só. Como corolário dos resultados temos o seguinte:

Corolário 5.31. Seja $r \in \mathbb{N}$ e $\mathbb{N}=\bigcup_{n=1}^{r} A_{r}$. Existe $j \in\{1, \ldots, r\}$ tal que:

1. Existe um conjunto infinito $H_{1} \subset \mathbb{N}$ tal que $F S\left(H_{1}\right) \subset A_{j}$.

2. Existe um conjunto infinito $H_{2} \subset \mathbb{N}$ tal que $F P\left(H_{2}\right) \subset A_{j}$.

3. $A_{j}$ contém progressões aritméticas arbitrariamente longas.

4. $A_{j}$ contém progressões geométricas arbitrariamente longas.

Demonstração. Seja $p \in(I P m \cdot) \cap \overline{(I P m+)}$. Como $p$ é ultrafiltro, existe $j \in\{1, \ldots, r\}$ tal que $A_{j} \in p$. Pelo Corolário 5.30 temos os itens (1) e (2).

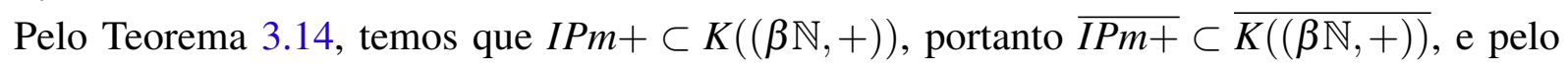


Teorema $5.14 A_{j}$ possui progressões aritméticas arbitrariamente longas. Do mesmo modo, $p \in(I P m \cdot) \subset \overline{K((\beta \mathbb{N}, \cdot))}$, portanto $A_{j}$ é sindético por partes em $(\mathbb{N}, \cdot)$ e pelo Teorema 5.14, temos que $A_{j}$ possui progressões geométricas arbitrariamente longas.

\subsection{Teorema de Hales-Jewett}

O Teorema de Hales-Jewett é um dos clássicos da Teoria de Ramsey e ilustra quão geral a definição de semigrupo pode ser.

O semigrupo usado nesta seção será o conjunto de palavras sobre um alfabeto com a operação de justaposição. Considere um conjunto finito $\Lambda=\left\{a_{1}, \ldots, a_{n}\right\}$, o qual chamaremos alfabeto e um elemento $v \notin \Lambda$ que denominado uma variável, definimos:

$$
W(\Lambda)=\Lambda^{<\omega}=\left\{\left(a_{1}, \ldots, a_{n}\right) \mid n \in \mathbb{N} \text { e } a_{i} \in A \text { para todo } i \in\{1, \ldots, n\}\right\} .
$$

Este é o conjunto de todas as palavras formadas pelos elementos do alfabeto $\Lambda$. Analogamente definimos $W(\Lambda, v)$, em que as palavras podem ser formadas pelo alfabeto $\Lambda$ e pela variável $v \notin \Lambda$. Denotamos também por $V(\Lambda) \subset W(\Lambda, v)$ o conjunto de palavras sobre $\Lambda \cup\{v\}$ em que $v$ ocorre ao menos uma vez, portanto $W(\Lambda, v)=W(\Lambda) \cup V(\Lambda)$. Dizemos que os elementos $\operatorname{de} W(\Lambda)$ são palavras constantes, enquanto que os elementos de $V(\Lambda)$ são palavras variáveis.

A operação usada será a de justaposição. Dadas duas palavras $\left(a_{1}, \ldots, a_{n}\right),\left(b_{1}, \ldots, b_{m}\right) \in W(\Lambda, v)$ temos

$$
\left(a_{1}, \ldots, a_{n}\right)^{\frown}\left(b_{1}, \ldots, b_{m}\right)=\left(a_{1}, \ldots, a_{n}, b_{1}, \ldots, b_{m}\right) .
$$

É fácil ver que $(W(\Lambda), \frown)$ é um semigrupo, de fato, é o semigrupo livre gerado por $\Lambda$.

Denotaremos uma palavra em $W(\Lambda, v)$ como $w(v)$, e se $a \in \Lambda \cup v$, então $w(a)$ é a palavra resultante de se substituir cada uma das ocorrências da variável $v$ por $a$ (dizemos que $w(a)$ é uma instância de $w(v)$ ). Se $a \in \Lambda$, então $w(a) \in W(\Lambda)$, e se $a=v$ então $w(a)=w(v)$.

Teorema 5.32. Seja $\Lambda$ um alfabeto finito, e considere o semigrupo $(W(\Lambda), \frown)$. Se $B \subset W(\Lambda) e ́$ sindético por partes, então existe uma palavra variável $w(v) \in V(\Lambda)$ tal que para todo a $\in \Lambda$, $w(a) \in B$.

Demonstração. Seja $\Lambda=\left\{a_{0}, \ldots, a_{l}\right\}$. Como no Teorema 5.14, temos que $Y=\chi_{t=0}^{l} \beta W(\Lambda)$ é uma compactificação de $X_{t=0}^{l} W(\Lambda)$.

Seja $I=\left\{\left(w\left(a_{0}\right), w\left(a_{1}\right), \ldots, w\left(a_{l}\right)\right) \mid w(v) \in V(\Lambda)\right\}$ e defina $E=I \cup\{(w, w, \ldots, w) \mid w \in W(\Lambda)\}$. Novamente temos que $\bar{E}$ é subsemigrupo de $Y, \bar{I}$ é ideal de $\bar{E}$ e $K(\bar{E})=\bar{E} \cap K(Y)$ e podemos provar que se $p \in K(\beta W(\Lambda))$, então $\Delta p \in \bar{E}$, da mesma forma que no teorema já referido.

Como $B$ é sindético por partes, existe $p \in \bar{B} \cap K(\beta W(\Lambda))$, portanto $p \in K(\bar{E}) \subset I$. Note que $U=$ $\chi_{t=0}^{l} \bar{B}$ é vizinhança de $\Delta p$, portanto $U \cap I \neq \emptyset$, então pela definição de $I$, existe $w(v) \in W(\Lambda, v)$ tal que $\left(w\left(a_{0}\right), \ldots, w\left(a_{l}\right)\right) \in U$.

Corolário 5.33 (Hales-Jewett). Sejam $\Lambda$ um alfabeto finito e $r \in \mathbb{N}$. Se $\bigcup_{i=1}^{r} A_{i}=S$ é uma partição de $S$, então existe $j \in\{1, \ldots, r\}$ e $w(v) \in W(\Lambda, v)$ tal que $\{w(a) \mid a \in \Lambda\} \subset A_{j}$. 
O que faremos a seguir é mostrar uma espécie de versão multidimensional do Teorema de Hales-Jewett. Ao invés de tratar de uma única palavra cujas instâncias estão contidas um uma mesma parte, teremos todas as instâncias de uma sequência infinita de palavras.

Este resultado foi originalmente publicado em Bergelson, Blass e Hindman (1994), tendo sido também incluído no Livro de Hindman e Strauss.

Definição 5.34. Seja $\left(s_{n}(v)\right)_{n \in \mathbb{N}}$ uma sequência em $W(\Lambda, v)$.

1. Uma sequência $\left(t_{n}\right)_{n \in \mathbb{N}}(v)$ de $W(\Lambda, v)$ é uma redução variável de $\left(s_{n}\right)_{n \in \mathbb{N}}$ se existe uma sequência $\left(n_{k}\right)_{k \in \mathbb{N}}$ de naturais com $n_{1}=1$ e uma sequência $\left(a_{n}\right)_{n \in \mathbb{N}}$ em $\Lambda \cup\{v\}$ (em que $v$ de fato ocorre) de modo que para todo $k \in \mathbb{N}$, temos

$$
\left.t_{k}(v)=s_{n_{k}}\left(a_{n_{k}}\right)\right\urcorner s_{n_{k}+1}\left(a_{n_{k}+1}\right)^{\frown} \ldots \frown s_{n_{k+1}-1}\left(a_{n_{k+1}-1}\right) .
$$

2. Uma sequência $\left(t_{n}\right)_{n \in \mathbb{N}}(v)$ de $W(\Lambda, v)$ é uma extração variável de $\left(s_{n}\right)_{n \in \mathbb{N}}$ se é uma redução variável de alguma subsequência de $\left(s_{n}(v)\right)_{n \in \mathbb{N}}$.

Exemplo 5.35. Considere $\Lambda=\{a, b, c\}, v \notin \Lambda$ uma variável e a sequência de palavras

$$
(a v, b v, c v, a v, b v, c v, \ldots)
$$

Alguns exemplos de reduções variáveis são:

$$
(a v b v c v, a v b v c v, \ldots)
$$

em que a sequência de naturais usada é $n_{k}=3 k$ e $a_{n}=v$ para todo $n$. Ou ainda:

$$
(a a, b b c c, a a b b c c, a a b b c c a a, \ldots) .
$$

Aqui a sequência de naturais usada é $n_{k}=n_{k-1}+k \operatorname{com} n_{0}=0$ e os $a_{n}$ repetem a sequência $(a, b, c)$.

A partir destas sequências podemos conseguir diferentes tipos de palavras.

Definição 5.36. Seja $\left(s_{n}(v)\right)_{n \in \mathbb{N}}$ uma sequência em $W(\Lambda, v)$.

1. Uma palavra $w \in W(\Lambda, v)$ é uma palavra reduzida de $\left(s_{n}(v)\right)_{n \in \mathbb{N}}$ se existem $k \in \mathbb{N}$ e $a_{1}, \ldots, a_{k} \in A \cup\{v\}$ tal que

$$
w=s_{1}\left(a_{1}\right) \frown \ldots s_{k}\left(a_{k}\right) .
$$

Se $w \in W(\Lambda, v)$ então $w$ é uma palavra reduzida variável, se $w \in S$ dizemos que $w$ é uma palavra reduzida constante.

2. Uma palavra $w \in W(\Lambda, v)$ é uma palavra extraída de $\left(s_{n}(v)\right)_{n \in \mathbb{N}}$ se é uma palavra reduzida de alguma subsequência de $\left(s_{n}(v)\right)_{n \in \mathbb{N}}$. Definimos analogamente ao caso anterior palavras extraídas variáveis e palavras extraídas constantes. 
Podemos definir esses conceitos analogamente para uma sequência finita $\left(s_{n}(v)\right)_{n=1}^{k}, k \in \mathbb{N}$.

Portanto uma palavra reduzida é simplesmente uma palavra formada pela concatenação de instâncias do segmento inicial.

Definição 5.37. Seja $\left(s_{n}(v)\right)_{n \in \mathbb{N}}$ um sequência em $W(\Lambda, v)$ e $k \in \mathbb{N} \cup\{\infty\}$. Para cada $m \in \mathbb{N}$ definimos:

1. $E\left(\left(s_{n}(v)\right)_{n=m}^{k}\right)$, o conjunto de todas as palavras extraídas de $\left(s_{n}(v)\right)_{n=m}^{k}$

2. $E V\left(\left(s_{n}(v)\right)_{n=m}^{k}\right)$, o conjunto das palavras variáveis extraídas de $\left(s_{n}(v)\right)_{n=m}^{k}$

3. $E C\left(\left(s_{n}(v)\right)_{n=m}^{k}\right)$, o conjunto das palavras constantes extraídas de $\left(s_{n}(v)\right)_{n=m}^{k}$.

Definimos também os seguintes conjuntos de $\beta W(\Lambda, v)$ :

$$
\begin{aligned}
& 1, \quad \mathscr{E}\left(\left(s_{n}(v)\right)_{n \in \mathbb{N}}\right)=\cap_{m \in \mathbb{N}} E\left(\left(\widehat{\left.s_{n}(v)\right)_{n=m}^{\infty}}\right) .\right. \\
& 2, \quad \mathscr{E} \mathscr{V}\left(\left(s_{n}(v)\right)_{n \in \mathbb{N}}\right)=\cap_{m \in \mathbb{N}} E V\left(\widehat{\left(s_{n}(v)\right)_{n=m}^{\infty}}\right) . \\
& 3, \quad \mathscr{E} \mathscr{C}\left(\left(s_{n}(v)\right)_{n \in \mathbb{N}}\right)=\cap_{m \in \mathbb{N}} E C\left(\widehat{\left(s_{n}(v)\right)_{n=m}^{\infty}}\right) .
\end{aligned}
$$

Lema 5.38. Seja $J$ um conjunto, $(D, \leq)$ um conjunto direcionado e $(S,+)$ um semigrupo. Seja $\left(T_{i}\right)_{i \in D}$ uma família de subconjuntos não vazios de $S$ em que se $i<j$, então $T_{j} \subset T_{i}$ e com a propriedade adicional de que para cada $i \in D$ e cada $x \in T_{i}$ existe $j \in D \operatorname{com} x+T_{i} \subset T_{j}$ e considere $Q=\bigcap_{i \in D} \overline{T_{i}}$, onde o fecho é tomado em $\beta S$. Sejam $\left(E_{i}\right)_{i \in D}$ e $\left(I_{i}\right)_{i \in D}$ famílias decrescentes de subconjuntos não vazios de $\chi_{j \in J} S$ satisfazendo:

1. Para cada $i \in D, I_{i} \subset E_{i} \subset \chi_{j \in J} T_{i}$.

2. Para cada $i \in I$ e cada $\vec{x} \in I_{i}$ existe $l \in D$ tal que $\vec{x}+E_{l} \subset I_{i}$.

3. Para cada $i \in I$ e cada $\vec{x} \in E_{i} \backslash I_{i}$ existe $l \in D$ tal que $\vec{x}+E_{l} \subset E_{i}$ e $\vec{x}+I_{l} \subset I_{i}$.

Se $Y=\chi_{j \in J} \beta S, E=\bigcap_{i \in D} \overline{E_{i}}$ e $I=\bigcap_{i \in D} \overline{I_{i}}$, os fechos em $E$ e $I$ tomados em $Y$, então $E$ é subsemigrupo de $\chi_{j \in J} Q$ e $I$ é ideal de $E$.

Demonstração. Pelo Lema 5.19 temos que $Q$ é um subsemigrupo. Comecemos mostrando que $E$ é subsemigrupo de $Y$.

Sejam $\vec{p}, \vec{q} \in E$. Considere $U \subset Y$ uma vizinhança de $\vec{p}+\vec{q}$ e $i \in D$ qualquer. Como $U$ é vizinhança de $\vec{p}+\vec{q}, V=\rho_{\vec{q}}^{-1}(U)$ é uma vizinhança de $\vec{p}$, e portanto existe $\vec{r} \in V \cap E_{i}$. Por hipótese existe $j \in D$ tal que $\vec{r}+E_{j} \subset E_{i}$. Por construção temos que $\vec{r}+\vec{q} \in U$, e como $\vec{r} \in E_{i} \subset \chi_{i \in D} S$ temos que $\lambda_{\vec{r}}$ é contínua, logo $W=\lambda_{\vec{r}}^{-1}(U)$ é vizinhança de $\vec{q}$ e assim existe $\vec{t} \in W \cap E_{j}$ e $\vec{r}+\vec{t} \in U \cap E_{i}$. Portanto $\vec{q}+\vec{q} \in \overline{E_{i}}$ para todo $i \in D$, $\operatorname{logo} \vec{p}+\vec{q} \in E$. 
Suponha agora que $\vec{p} \in I$ e $\vec{q} \in E$, vamos mostrar que $\vec{p}+\vec{q} \in I$.

Considere $U \subset Y$ uma vizinhança de $\vec{p}+\vec{q}$ e $i \in D$ qualquer. Como $U$ é vizinhança de $\vec{p}+\vec{q}$, $V=\rho_{\vec{q}}^{-1}(U)$ é uma vizinhança de $\vec{p}$, e portanto existe $\vec{r} \in V \cap I_{i}$. Por hipótese existe $j \in D$ tal que $\vec{r}+E_{j} \subset I_{i}$. Por construção temos que $\vec{r}+\vec{q} \in U$, e como $\vec{r} \in I_{i} \subset X_{i \in D} S$ temos que $\lambda_{\vec{r}}$ é contínua, $\operatorname{logo} W=\lambda_{\vec{r}}^{-1}(U)$ é vizinhança de $\vec{q}$ e assim existe $\vec{t} \in W \cap E_{j}$ e $\vec{r}+\vec{t} \in U \cap I_{i}$. Mostramos então $\vec{q}+\vec{q} \in \overline{I_{i}}$ para todo $i \in D, \operatorname{logo} \vec{p}+\vec{q} \in I$.

Por fim suponha que $\vec{p} \in E$ e $\vec{q} \in I$, vamos mostrar que $\vec{p}+\vec{q} \in I$.

Considere $U \subset Y$ uma vizinhança de $\vec{p}+\vec{q}$ e $i \in D$ qualquer. Como $U$ é vizinhança de $\vec{p}+\vec{q}$, $V=\rho_{\vec{q}}^{-1}(U)$ é uma vizinhança de $\vec{p}$, e portanto existe $\vec{r} \in V \cap E_{i}$. Por hipótese existe $j \in D$ tal que $\vec{r}+I_{j} \subset I_{i}$. Por construção temos que $\vec{r}+\vec{q} \in U$, e como $\vec{r} \in E_{i} \subset X_{i \in D} S$ temos que $\lambda_{\vec{r}}$ é contínua, $\operatorname{logo} W=\lambda_{\vec{r}}^{-1}(U)$ é vizinhança de $\vec{q}$ e assim existe $\vec{t} \in W \cap I_{j}$ e $\vec{r}+\vec{t} \in U \cap I_{i}$. Mostramos então $\vec{q}+\vec{q} \in \overline{I_{i}}$ para todo $i \in D$, $\operatorname{logo} \vec{p}+\vec{q} \in I$.

Lema 5.39. Seja $\left(s_{n}\right)_{n \in \mathbb{N}}$ uma sequência em $W(\Lambda, v)$, então $\mathscr{E}\left(\left(s_{n}\right)_{n=1}^{\infty}\right.$ é um subsemigrupo compacto de $\beta W(\Lambda, v), \mathscr{E} \mathscr{V}\left(\left(s_{n}\right)_{n \in \mathbb{N}}\right)$ é um ideal de $\mathscr{E}\left(\left(s_{n}\right)_{n \in \mathbb{N}}\right)$. Se $\Lambda \neq \emptyset$, então $\mathscr{E} \mathscr{C}\left(\left(s_{n}\right)_{n \in \mathbb{N}}\right)$ é um subsemigrupo compacto de $\beta W(\Lambda, v)$

Demonstração. Considere $J=\{1\}, D=\mathbb{N}$ e para todo $n \in \mathbb{N}, T_{n}=W(\Lambda, v)$. Para cada $m \in \mathbb{N}$, defina $E_{m}=E\left(\left(s_{n}(v)\right)_{n=m}^{\infty}\right), I_{m}=E V\left(\left(s_{n}(v)\right)_{n=m}^{\infty}\right)$ e $C_{m}=E C\left(\left(s_{n}(v)\right)_{n=m}^{\infty}\right)$. Vamos mostrar que estamos nas hipóteses do lema anterior.

É claro pela definição que $I_{m} \subset E_{m} \subset W(\Lambda, v)=T_{m}$ para todo $m \in \mathbb{N}$, portanto o item (1) é satisfeito.

Para o item (2) sejam $m \in \mathbb{N}$ e $w \in I_{m}$. Como $w \in I_{m}$, existem $t \in \mathbb{N}, n_{1}, \ldots, n_{t} \in \mathbb{N}$ com $n_{1} \geq m$ e $a_{1}, \ldots, a_{t} \in A \cup\{v\}$ com ao menos um $a_{i}=v$ tal que

$$
w=s_{n_{1}}\left(a_{1}\right) \frown s_{n_{2}}\left(a_{2}\right) \frown \ldots \frown s_{n_{t}}\left(a_{t}\right)
$$

Se $u \in E_{n_{t}+1}$ então $u=s_{k_{1}}\left(b_{1}\right)^{\frown} \ldots \frown s_{k_{r}}\left(b_{r}\right)$ com $k_{1} \geq n_{t}+1$ e $b_{i} \in A \cup\{v\}$ para cada $i \in$ $\{1, \ldots, r\}$, e assim é claro que $w^{\frown} u=\left(s_{n_{1}}\left(a_{1}\right)^{\frown} \ldots \frown s_{n_{t}}\left(a_{t}\right)\right)^{\frown}\left(s_{k_{1}}\left(b_{1}\right)^{\frown} \ldots \frown s_{k_{r}}\left(b_{r}\right)\right) \in I_{m}$.

Note que se repetirmos a mesma demonstração utilizando $a_{1}, \ldots, a_{t} \in \Lambda$ conseguimos o item (3), e assim conseguimos a primeira parte.

Suponha agora que $\Lambda \neq \emptyset$, pelo Lema 5.19 basta mostrar que para todo $k \in \mathbb{N}$ e $w \in C_{k}$ existe $m \in \mathbb{N}$ com $w^{\frown} C_{m} \subset E C\left(\left(s_{n}(v)\right)_{n=k}^{\infty}\right)$. Novamente essa demonstração é idêntica à verificação anterior para o item (2), porém utilizando constantes $a_{1}, \ldots, a_{t} \in \Lambda$.

Estamos em condição de demonstrar o teorema principal desta seção.

Teorema 5.40. Seja $\Lambda$ um alfabeto finito não vazio e $\left(s_{n}(v)\right)_{n \in \mathbb{N}}$ uma sequência em $W(\Lambda, v)$. Sejam $V(\Lambda)=\bigcup_{i=1}^{r_{1}} A_{i} e W(\Lambda)=\bigcup_{i=1}^{r_{2}} B_{i}$ partições finitas. Existem $j_{1} \in\left\{1, \ldots, r_{1}\right\}, j_{2} \in\left\{1 \ldots, r_{2}\right\}$ e uma extração variável $\left(w_{n}(v)\right)_{n \in \mathbb{N}}$ de $\left(s_{n}(v)\right)_{n \in \mathbb{N}}$ tal que $A_{j_{1}}$ contém todas as palavras extraídas variáveis de $\left(w_{n}(v)\right)_{n \in \mathbb{N}}$ e $B_{j_{2}}$ contém todas as palavras extraídas constantes de $\left(w_{n}(v)\right)_{n \in \mathbb{N}}$. 
Demonstração. Pelo lema anterior $\mathscr{E} \mathscr{C}\left(\left(s_{n}(v)\right)_{n \in \mathbb{N}}\right) \subset \mathscr{E}\left(\left(s_{n}(v)\right)_{n \in \mathbb{N}}\right)$ é um subsemigrupo compacto de $\beta W(\Lambda, v)$, portanto contém um idempotente minimal $p$. Note que $p$ é um idempotente de $\mathscr{E}\left(\left(s_{n}(v)\right)_{n \in \mathbb{N}}\right)$, porém não necessariamente minimal. Tome então $q \leq p$ minimal em $\mathscr{E}\left(\left(s_{n}(v)\right)_{n \in \mathbb{N}}\right)$ e como $\mathscr{E} \mathscr{V}\left(\left(s_{n}(v)\right)_{n \in \mathbb{N}}\right)$ é um ideal de $\mathscr{E}\left(\left(s_{n}(v)\right)_{n \in \mathbb{N}}\right)$, temos que

$$
q \in K(\beta W(\Lambda, v)) \subset \mathscr{E} \mathscr{V}\left(\left(s_{n}(v)\right)_{n \in \mathbb{N}}\right)
$$

Para cada $a \in \Lambda$, considere a função avaliação $\varphi_{a}: W(\Lambda, v) \rightarrow W(\Lambda)$ dada por $w(v)=w(a)$. É imediato que $\varphi_{a}$ é homomorfismo, portanto sua extensão contínua $\tilde{\varphi}_{a}: \beta W(\Lambda, v) \rightarrow \beta W(\Lambda)$ é um homomorfismo (Lema 5.22). Vamos mostrar que para todo $a \in \Lambda, \tilde{\varphi}_{a}(q)=p$.

Por ser homomorfismo temos que $\tilde{\varphi}_{a}(p)$ e $\tilde{\varphi}_{a}(q)$ são idempotentes e que $\tilde{\varphi}_{a}(q) \leq \tilde{\varphi}_{a}(p)$. Além disso, $\tilde{\varphi}_{a}$ é a função identidade em $W(\Lambda)$ e $p \in \beta W(\Lambda)$, portanto $\tilde{\varphi}_{a}(p)=p$. Mas então $\tilde{\varphi}_{a}(q) \leq$ $\tilde{\varphi}_{a}(p)=p$, logo, por minimalidade de $p$, basta mostrarmos que $\tilde{\varphi}(q) \in \mathscr{E} \mathscr{C}\left(\left(s_{n}(v)\right)_{n \in \mathbb{N}}\right)$.

Seja $m \in \mathbb{N}$. Pela definição de $\mathscr{E} \mathscr{V}\left(\left(s_{n}(v)\right)_{n \in \mathbb{N}}\right)$ vemos que

$$
E V\left(s_{n}(v)_{n=m}^{\infty}\right) \in q \Rightarrow \tilde{\varphi}_{a}\left(E V\left(s_{n}(v)_{n=m}^{\infty}\right)\right) \in \tilde{\varphi}_{a}(q)
$$

mas

$$
\tilde{\varphi}_{a}\left(E V\left(s_{n}(v)_{n=m}^{\infty}\right)\right) \subset E C\left(s_{n}(v)_{n=m}^{\infty}\right),
$$

$\operatorname{logo} E C\left(s_{n}(v)_{n=m}^{\infty}\right) \in \tilde{\varphi}_{a}(q)$ para todo $m \in \mathbb{N}$, o que demonstra nossa afirmação.

Seja $D \in\left\{A_{1}, \ldots, A_{j_{1}}\right\}$ pertencente a $q$ e $C \in\left\{B_{1}, \ldots, B_{j_{2}}\right\}$ pertencente a $p$ e defina os seguintes conjuntos:

$$
\begin{gathered}
C^{\prime}=\left\{t \in C \mid, C \in t^{\frown} p\right\} \cap\left\{t \in C \mid D \in t^{\frown} q\right\} \mathrm{e} \\
D^{\prime}=\left\{t(v) \in D \mid D \in t(v)^{\frown} p\right\} \cap\left\{t(v) \in D \mid D \in t(v)^{\frown} q\right\} .
\end{gathered}
$$

Vamos mostrar que $\left\{t \in C \mid C \in t^{\frown} p\right\} \in p$.

$$
\begin{gathered}
t^{\frown p} p=\left\{B \subset W(\Lambda, v) \mid\left\{r \in W(\Lambda, v) \mid t^{\frown} r \in B\right\} \in p\right\} \text { portanto } \\
\left\{t \in C \mid C \in t^{\frown} p\right\} \\
=\left\{t \in C \mid C \in\left\{B \subset W(\Lambda, v) \mid\left\{r \in W(\Lambda, v) \mid t^{\frown} r \in B\right\} \in p\right\}\right\} \\
=\left\{t \in C \mid\left\{r \in W(\Lambda, v) \mid t^{\frown} r \in C\right\} \in p\right\} \\
=\left\{t \in W(\Lambda, v) \mid\left\{r \in W(\Lambda, v) \mid t^{\frown} r \in C\right\} \in p\right\} \cap C
\end{gathered}
$$

Note que $C \in p=p^{\frown} p, \operatorname{logo}\left\{t \in W(\Lambda, v) \mid\left\{r \in W(\Lambda, v) \mid t^{\frown} r \in C\right\} \in p\right\} \in p$, assim temos que os dois termos da interseção acima são elementos de $p$ e, consequentemente, $\left\{t \in C \mid C \in t^{\curvearrowleft} p\right\} \in p$. Analogamente, usando que $q=q^{\frown} q=p^{\frown} q=q^{\frown} p$ podemos mostrar que $\left\{t \in C \mid D \in t^{\frown} q\right\} \in p$, $\left\{t(v) \in D \mid D \in t(v)^{\frown} p\right\} \in q$ e $\left\{t(v) \in D \mid D \in t(v)^{\wedge} q\right\} \in q$, e portanto que $C^{\prime} \in p$ e $D^{\prime} \in q$.

Por falta de notação específica denotaremos, como no produto, o conjunto

$$
t^{-1} V:=\left\{r \in W(\Lambda, v) \mid t^{\frown} r \in V\right\} \text { para } V \subset W(\Lambda, v) \text { e } t \in W(\Lambda, v)
$$


Vamos mostrar que para todo $x \in C^{\prime}$ e $y \in D^{\prime}, x^{-1} C^{\prime} \in p, x^{-1} D^{\prime} \in q, y^{-1} D^{\prime} \in p$ e $y^{-1} D^{\prime} \in q$.

Seja $y \in D^{\prime}$. Por definição temos que $y^{\wedge} q \in \widehat{D}$, então $\lambda_{y}^{-1}\left(\rho_{p}^{-1}(\widehat{D})\right)$ e $\lambda_{y}^{-1}\left(\rho_{q}^{-1}(\widehat{D})\right)$ são vizinhanças de $q$, pois $q$ é idempotente, $q \leq p$,

$$
\begin{aligned}
& \lambda_{y}^{-1}\left(\rho_{q}^{-1}(\widehat{D})\right)=\left\{r \in \beta W(\Lambda, v) \mid y^{\frown} r^{\frown} q \in \widehat{D}\right\} \mathrm{e} \\
& \lambda_{y}^{-1}\left(\rho_{p}^{-1}(\widehat{D})\right)=\left\{r \in \beta W(\Lambda, v) \mid y^{\frown} r^{\frown} p \in \widehat{D}\right\} .
\end{aligned}
$$

Além disso, como $y \in D^{\prime}$, temos que $y^{-1} D \in q$.

Note que $y^{-1} D^{\prime}=y^{-1} D \cap \lambda_{y}^{-1}\left(\rho_{q}^{-1}(\widehat{D})\right) \cap \lambda_{y}^{-1}\left(\rho_{p}^{-1}(\widehat{D})\right)$, pois

$$
\begin{aligned}
y^{-1} D^{\prime} & =\left\{t \in W(\Lambda, v) \mid y^{\frown} t \in D^{\prime}\right\} \\
& =\left\{t \in W(\Lambda, v) \mid y^{\frown} t \in\left\{r \in D \mid D \in r^{\frown} p \text { e } D \in r^{\frown} q\right\}\right\} \\
& =\left\{t \in W(\Lambda, v) \mid y^{\frown} t \in D \text { e } D \in y^{\urcorner} t^{\frown} p \text { e } D \in y^{\urcorner} t^{\frown} q .\right\}
\end{aligned}
$$

Então $y^{-1} D^{\prime} \in q$, já que cada conjunto da interseção é um elemento de $q$. Do mesmo modo podemos ver que $p$ pertence a cada um dos conjuntos, logo $y^{-1} D^{\prime} \in p$. De modo análogo concluímos que $x^{-1} D^{\prime}=x^{-1} D \cap \lambda_{x}^{-1}\left(\rho_{q}^{-1}(\widehat{D})\right) \cap \lambda_{x}^{-1}\left(\rho_{p}^{-1}(\widehat{D})\right) \in q$ e $x^{-1} C^{\prime}=x^{-1} c \cap \lambda_{x}^{-1}\left(\rho_{q}^{-1}(\widehat{D})\right) \cap$ $\lambda_{x}^{-1}\left(\rho_{p}^{-1}(\widehat{C})\right) \in p$. A afirmação está, assim, demonstrada.

Dados $t, r \in E\left(\left(s_{n}(v)\right)_{n=1}^{\infty}\right)$, diremos que $t \preccurlyeq r$ se existir $k \in \mathbb{N}$ de modo que $t \in E\left(\left(s_{n}(v)\right)_{n=1}^{k}\right)$ e $r \in E\left(\left(s_{n}(v)\right)_{n=k+1}^{\infty}\right)$, ou seja, se $t$ é uma palavra extraída de um segmento inicial da sequência e $r$ uma palavra extraída do restante da sequência. Note que

$$
\left\{r \in E\left(\left(s_{n}(v)\right)_{n \in \mathbb{N}}\right) \mid t \preccurlyeq r\right\}=E\left(\left(s_{n}(v)\right)_{n=k+1}^{\infty}\right) \text { em que } t \in E\left(\left(s_{n}(v)\right)_{n=1}^{k}\right) \text {. }
$$

Como $q \in \bigcap_{n \in \mathbb{N}} E\left(\widehat{\left(s_{n}(v)\right)_{n=l}^{\infty}}\right)$, temos que $E\left(\left(s_{n}(v)\right)_{n=l}^{\infty}\right) \in q$ para todo $l \in \mathbb{N}$, portanto $\{r \in$ $\left.E\left(\left(s_{n}(v)\right)_{n \in \mathbb{N}}\right) \mid t \preccurlyeq r\right\} \in q$.

Vamos agora construir uma extração variável $\left(w_{n}(v)\right)_{n=1}^{\infty}$ de $\left(s_{n}(v)\right)_{n=1}^{\infty}$ tal que $E C\left(\left(w_{n}(v)\right)_{n=1}^{\infty}\right) \subset$ $C^{\prime}$ e $E V\left(\left(w_{n}(v)\right)_{n=1}^{\infty}\right) \subset D^{\prime}$, ou seja, todas as extrações constantes de $\left(w_{n}(v)\right)_{n=1}^{\infty}$ estão em $C^{\prime} \subset C$ e todas as extrações variáveis de $\left(w_{n}(v)\right)_{n=1}^{\infty}$ estão em $D^{\prime} \subset D$, e assim teremos o resultado.

Note que $C^{\prime} \in p$ e para todo $a \in A$ temos que $p=\varphi_{a}(q)$, o que implica $\varphi_{a}^{-1}\left(C^{\prime}\right) \in q$ para todo $a \in A$ e $D^{\prime} \in q$, então o conjunto $D^{\prime} \cap \bigcap_{a \in A} \varphi_{a}\left(C^{\prime}\right) \in q$ e, em particular, é infinito. Tome $w_{1}(v) \in D^{\prime} \cap \bigcap_{a \in A} \varphi_{a}\left(C^{\prime}\right)$.

Suponha que já tenhamos escolhido $w_{1}(v), \ldots, w_{k-1}(v)$ com $w_{i} \preccurlyeq w_{i+1}$ para $i \in\{1, \ldots, k-1\}$, $E C\left(\left(w_{n}(v)\right)_{n=1}^{k}\right) \subset C^{\prime}$ e $E V\left(\left(w_{n}(v)\right)_{n=1}^{k}\right) \subset D^{\prime}$. Demonstramos então que:

$$
\begin{gathered}
\bigcap_{n=1}^{k-1}\left\{t \in E\left(\left(s_{n}(v)\right)_{n=1}^{k-1}\right) \mid w_{n}(v) \preccurlyeq t\right\} \in q \forall n \in\{1, \ldots, k-1\} ; \\
t^{-1} D^{\prime} \in q \forall t \in E V\left(\left(w_{n}(v)\right)_{n=1}^{k-1}\right),
\end{gathered}
$$

pois $E V\left(\left(w_{n}(v)\right)_{n=1}^{k-1}\right) \subset D^{\prime}$ pela hipótese de indução;

$$
\varphi_{a}^{-1}\left(t^{-1}\left(D^{\prime}\right)\right) \in q \forall a \in A \text { e } \forall t \in E V\left(\left(w_{n}(v)\right)_{n=1}^{k-1}\right) ;
$$




$$
\text { e } t^{-1}\left(C^{\prime}\right) \in q \forall t \in E C\left(\left(w_{n}(v)\right)_{n=1}^{k-1}\right) .
$$

Como são finitos conjuntos, sua interseção é infinita, pois pertence a $q$, logo podemos escolher um elemento $w_{k}(v)$ nesta interseção que seja distinto de todas as escolhas anteriores. Construímos então $w_{k}(v)$ satisfazendo:

1. $w_{k-1}(v) \preccurlyeq w_{k}(v)$.

2. $w_{k}(v) \in D^{\prime}$.

3. $w_{k}(v) \in \varphi_{a}^{-1}\left(C^{\prime}\right)$ para todo $a \in A$.

4. $w_{k}(v) \in t^{-1} D^{\prime}$ para todo $t \in E V\left(\left(w_{i}(v)\right)_{i=1}^{k-1}\right)$.

5. $w_{k}(v) \in \varphi_{a}^{-1}\left(t^{-1}\left(D^{\prime}\right)\right)$ para todo $t \in E V\left(\left(w_{i}(v)\right)_{i=1}^{k-1}\right)$ e todo $a \in A$.

6. $w_{k}(v) \in t^{-1} D^{\prime}$ para todo $t \in E C\left(\left(w_{i}(v)\right)_{i=1}^{k-1}\right)$.

7. $w_{k}(v) \in \varphi_{a}^{-1}\left(t^{-1}\left(C^{\prime}\right)\right)$ para todo $t \in E C\left(\left(w_{i}(v)\right)_{i=1}^{k-1}\right)$ e todo $a \in A$.

Para concluir a indução precisamos mostrar que $E C\left(\left(w_{i}(v)\right)_{i=1}^{k}\right) \subset C^{\prime}$ e $E V\left(\left(w_{i}(v)\right)_{i=1}^{k}\right) \subset D^{\prime}$. Seja $u \in E C\left(\left(w_{i}(v)\right)_{i=1}^{k}\right)$, então existe $l \in \mathbb{N}, n_{1}, \ldots, n_{l} \in \mathbb{N}$ e $a_{1}, \ldots, a_{l} \in A$ de modo que $u=w_{n_{1}}\left(a_{1}\right)^{\frown} w_{n_{2}}\left(a_{2}\right)^{\frown} \ldots{ }^{\wedge} w_{n_{l}}\left(a_{l}\right)$. Se $n_{l}<k$ a hipótese de indução garante que $u \in C^{\prime}$, então suponha que $n_{l}=k$. Se $l=1$, então $u=w_{k}\left(a_{1}\right) \in C^{\prime}$ ) pela condição (3), se $l>1$ então a condição (7) diz que $u \in C^{\prime}$.

Tome agora $u \in E V\left(\left(w_{i}(v)\right)_{i=1}^{k}\right)$, então $u=w_{n_{1}}\left(a_{1}\right)^{\frown} w_{n_{2}}\left(a_{2}\right)^{\frown} \ldots \frown w_{n_{l}}\left(a_{l}\right) \operatorname{com} a_{1}, \ldots, a_{l} \in$ $A \cup\{v\}$ sendo que existe algum $j \in\{1, \ldots, l\}$ tal que $a_{j}=v$. Novamente, se $n_{l}<k, u \in D^{\prime}$ pela hipótese de indução e se $n_{l}=k$ mas $l=1$, então $u \in D^{\prime}$ pela condição (2). Seja então $n_{l}=k$ e $l>1$, analisemos os três casos possíveis: $a_{l} \in A ; a_{l}=v$ e $\left\{a_{1}, \ldots, a_{l-1}\right\} \subset A$; e $a_{l}=v$ e $v \in\left\{a_{1}, \ldots, a_{l-1}\right\}$. No primeiro caso usamos a condição (5), no segundo a condição (6) e por último a condição (4).

Por fim, note que a condição (1) nos garante que $\left(w_{n}(v)\right)_{n \in \mathbb{N}}$ é uma extração variável de $\left(s_{n}(v)\right)_{n \in \mathbb{N}}$.

O próximo corolário é uma versão deste mesmo teorema, porém usando reduções ao invés de extrações. Note que a existência de uma redução variável da sequência original é uma condição mais forte do que a existência de uma extração variável, porém a condição de todas as palavras reduzidas de uma sequência pertencerem a um conjunto é mais fraca do que todas as palavras extraídas pertencerem a um conjunto. Faremos então uma troca, impondo mais restrições sobre a extração e menos sobre a condição de homogeneidade.

Corolário 5.41. Seja $\Lambda$ um alfabeto finito não vazio e $\left(s_{n}(v)\right)_{n \in \mathbb{N}}$ uma sequência em $W(\Lambda, v)$. Sejam $V(\Lambda)=\bigcup_{i=1}^{r_{1}} A_{i}$ e $W(\Lambda)=\bigcup_{i=1}^{r_{2}} B_{i}$ partições finitas. Existem $j_{1} \in\left\{1, \ldots, r_{1}\right\}, j_{2} \in\left\{1 \ldots, r_{2}\right\}$ e uma redução variável $\left(w_{n}(v)\right)_{n \in \mathbb{N}}$ de $\left(s_{n}(v)\right)_{n \in \mathbb{N}}$ tal que $A_{j_{1}}$ contém todas as palavras reduzidas variáveis de $\left(w_{n}(v)\right)_{n \in \mathbb{N}}$ e $B_{j_{2}}$ contém todas as palavras reduzidas constantes de $\left(w_{n}(v)\right)_{n \in \mathbb{N}}$. 
Demonstração. Defina a função $\varphi: W(\Lambda, v) \rightarrow W(\Lambda, v)$ de modo que se $w=a_{1}, \ldots a_{k}$, então

$$
\varphi(w)=\varphi\left(a_{1} \ldots a_{k}\right)=s_{1}\left(a_{1}\right) \frown s_{2}\left(a_{2}\right)^{\frown} \ldots \frown s_{k}\left(A_{k}\right) .
$$

Note que $\varphi(V(\Lambda)) \subset V(\Lambda)$ e $\varphi(W(\Lambda)) \subset W(\Lambda)$. Definimos também, para cada $i \in\left\{1, \ldots, r_{1}\right\}$ os conjuntos $A_{i}^{\prime}=\varphi^{-1}\left(A_{i}\right)$ e para cada $i \in\left\{1, \ldots, r_{2}\right\}$ os conjuntos $B_{i}^{\prime}=\varphi^{-1}\left(B_{i}\right)$, portanto $W(\Lambda)=\bigcup_{i=1}^{j_{1}} A_{i}^{\prime}$ e $V(\Lambda)=\bigcup_{i=1}^{j_{1}} B_{i}^{\prime}$. Aplique o teorema anterior para essas partições e a sequência de palavras $(v, v, v, v, v \ldots)$, então existem uma extração variável $\left(t_{n}(v)\right)_{n \in \mathbb{N}}$ (note que qualquer sequência de palavras variáveis é uma extração variável desta sequência), $l_{1} \in\left\{1, \ldots, r_{1}\right\}$ e $l_{2} \in\left\{1, \ldots, r_{2}\right\}$ tal que as palavras extraídas constantes de $\left(t_{n}(v)\right)_{n \in \mathbb{N}}$ estão em $A_{j_{1}}^{\prime}$ e as palavras extraídas variáveis estão em $B_{j_{2}}^{\prime}$.

Para cada $n \in \mathbb{N}$, escrevemos a palavra $t_{n}(v)$ como $a_{1}^{n} a_{2}^{n} \ldots a_{k_{n}}^{n}$, portanto $k_{n}$ é o comprimento da palavra $t_{n}(v)$ e definimos $h_{n}=\sum_{i=1}^{n-1} k_{i}$. Vamos definir a redução $\left(w_{n}(v)\right)_{n \in \mathbb{N}}$ de $\left(s_{n}(v)\right)_{n \in \mathbb{N}}$ como

$$
w_{n}(v)=s_{h_{n}+1}\left(a_{1}^{n}\right)^{\frown} s_{h_{n}+2}\left(a_{2}^{n}\right)^{\frown} \ldots \frown s_{h_{n}+k_{n}}\left(a_{k_{n}}^{n}\right) .
$$

Por construção, dados $b_{1}, \ldots, b_{n} \in A \cup\{v\}$, temos que

$$
\left.\left.\varphi\left(t_{1}\left(b_{1}\right)\right\urcorner t_{2}\left(b_{2}\right) \frown \ldots t_{n}\left(b_{n}\right)\right)=w_{1}\left(b_{1}\right)\right\urcorner w_{2}\left(b_{2}\right)^{\frown} \ldots \frown w_{n}\left(b_{n}\right) .
$$

Seja $b_{1}, \ldots, b_{n} \in A$, então

$$
t_{1}\left(b_{1}\right)^{\frown} \ldots \frown t_{n}\left(b_{n}\right) \in A_{j_{1}}^{\prime} \Rightarrow \varphi\left(t_{1}\left(b_{1}\right)^{\frown} \ldots \frown t_{n}\left(b_{n}\right)\right)=w_{1}\left(b_{1}\right)^{\frown} w_{2}\left(b_{2}\right)^{\frown} \ldots \frown w_{n}\left(b_{n}\right) \in A_{j_{1}} .
$$

Analogamente, se $b_{1}, \ldots, b_{n} \in A \cup\{v\}$, então

$$
t_{1}\left(b_{1}\right)^{\frown} \ldots \frown t_{n}\left(b_{n}\right) \in B_{j_{2}}^{\prime} \Rightarrow \varphi\left(t_{1}\left(b_{1}\right)^{\frown} \ldots \frown t_{n}\left(b_{n}\right)\right)=w_{1}\left(b_{1}\right)^{\frown} w_{2}\left(b_{2}\right)^{\frown} \ldots \frown w_{n}\left(b_{n}\right) \in B_{j_{2}} .
$$

\subsection{Recorrência e Teoria de Ramsey}

Nesta seção mostraremos que alguns dos resultados de Teoria de Ramsey podem ser reformulados na linguagem de dinâmica topológica. Veremos que, em qualquer sistema dinâmico sobre um espaço métrico compacto, podemos garantir a existência de um ponto cuja recorrência segue a regularidade das famílias regulares por partições, como a dos conjuntos AP-rich ou a família dos IP-set's.

Começaremos pela versão de recorrência do Teorema de Van der Waerden.

Proposição 5.42. Se $(X, T)$ é um sistema dinâmico em que $X$ é um espaço métrico compacto, então, para todo $\varepsilon>0$, existe $x \in X$ de modo que o conjunto

$$
\left\{n \in \mathbb{N} \mid d\left(x, T^{n}(x)\right)<\varepsilon\right\}
$$

é AP-rich. 
Demonstração. Considere um sistema dinâmico $(X, T)$ em que $X$ é um espaço métrico compacto e fixe $\varepsilon>0$.

Para cada $x \in X$, defina a bola aberta $B_{x}$ de centro $x$ e raio $\frac{\varepsilon}{2}$, então $\left\{B_{x} \mid x \in X\right\}$ é uma cobertura aberta de $X$. Como $X$ é compacto, existe uma subcobertura finita $\left\{B_{1}, \ldots, B_{r}\right\} \subset\left\{B_{x} \mid x \in X\right\}$. Fixe um elemento $y \in X$ e, para cada $i \in\{1, \ldots, r\}$, defina o conjunto $A_{i} \subset \mathbb{N}$ como $n \in A_{i} \Leftrightarrow$ $T^{n}(y) \in B_{i}$ e note que $\mathbb{N}=\bigcup_{i=1}^{r} A_{i}$.

Pelo Teorema de Van der Waerden (5.14), a família dos conjuntos AP-rich em $\mathbb{N}$ é fracamente regular por partições, portanto existe $j \in\{1, \ldots, r\}$ tal que $A_{j}$ é AP-rich. Mas então, para todo $m, n \in A_{j}$, temos que $d\left(T^{m}(y), T^{n}(y)\right)<\varepsilon$.

Defina $m=\min \left\{A_{j}\right\}$ e $x=T^{m}(y)$, então para todo $n \in-m+A_{j}$,

$$
d\left(x, T^{n}(x)\right)=d\left(T^{m}(y), T^{n}\left(T^{m}(y)\right)\right)=d\left(T^{m}(y), T^{n+m}(y)\right)<\varepsilon,
$$

pois $n \in-m+A_{j}$, logo $m \in A_{j}$ e $n+m \in A$.

Como $A_{j}$ é AP-rich, temos que $-m+A_{j}$ é AP-rich e, como $-m+A_{j} \subset\left\{n \in \mathbb{N} \mid d\left(x, T^{n}(x)\right)<\varepsilon\right\}$, temos o resultado.

A proposição acima é, portanto, uma consequência do Teorema de Van der Waerden. O que mostraremos a seguir é que eles são, de fato, equivalentes.

Para isso consideraremos o sistema dinâmico

$$
\Omega=\{1, \ldots, r\}^{\mathbb{N}} \text { com a métrica } d(\gamma, \xi)=\frac{1}{m}, m=\min \{k \in \mathbb{N} \mid \gamma(k) \neq \xi(k)\}
$$

e o shift map $T: \Omega \rightarrow \Omega$, ou seja $T(\xi)(n)=\xi(n+1)$. O argumento para o caso de semigrupos arbitrários falha, pois implicitamente utilizamos a ordem usual do conjunto dos naturais na definição da métrica.

Teorema 5.43. Suponha que para todo sistema dinâmico $(X, T)$, em que $X$ é um espaço métrico compacto, e para todo $\varepsilon>0$, existe $x \in X$ de modo que o conjunto

$$
\left\{n \in \mathbb{N} \mid d\left(x, T^{n}(x)\right)<\varepsilon\right\}
$$

é AP-rich. Então a família dos conjuntos AP-rich de $\mathbb{N}$ é fracamente regular por partições.

Demonstração. Sejam $k, r \in \mathbb{N}$ e $\mathbb{N}=\bigcup_{i=1}^{r} A_{i}$ uma partição finita de $\mathbb{N}$. Considere $\Omega, d$ e $T$ como descrito acima. Defina a função $f_{0}: \mathbb{N} \rightarrow\{1 \ldots, r\}$ como $f_{0}(n)=i$ se, e somente se, $n \in A_{i}$ e note que $f_{0} \in \Omega$. Defina $X=\overline{\left\{T^{n}\left(f_{0}\right) \mid n \in \mathbb{N}\right\}}$. Por hipótese, tomando $\varepsilon=1$, existe $x \in X$ tal que $\left\{n \in \mathbb{N} \mid d\left(x, T^{n}(x)\right)<1\right\}$ é um conjunto AP-rich, portanto existem $a, d \in \mathbb{N}$ de modo que $d\left(x, T^{a+i d}(x)\right)<1$ para todo $i \in\{1, \ldots, k\}$. Lembre-se que $x$ é uma função e $T$ é o shift map, então concluímos que

$$
x(1)=x(a+1)=x(a+1+d)=\cdots=x(a+1+k d) .
$$

Note que $x \in X=\overline{\left\{T^{n}\left(f_{0}\right) \mid n \in \mathbb{N}\right\}}$, portanto existe $m \in \mathbb{N}$ tal que $d\left(x, T^{m}\left(f_{0}\right)\right)<\frac{1}{a+k d+2}$, portanto $x(n)=f(n+m) \forall n \leq a+1+k d$. Note então que se $j=f(m)$, então $\{m, m+n+1, m+$ $2 n+1, \ldots, m+(k-1) n+1\} \subset A_{j}$. 
Um ponto é recorrente se para toda vizinhança $V$ de $x$ o conjunto $\left\{n \in \mathbb{N} \mid T^{n}(x) \in V\right\}$ é infinito, mas, mais do que isso, mostramos que esses infinitos retornos possuem uma regularidade, neste caso eles contém progressões aritméticas arbitrariamente longas.

O Teorema de Hindman tem uma relação ainda mais profunda com a recorrência de sistemas dinâmicos, pois a recorrência de qualquer ponto se dá por um IP-set e qualquer $H \subset \mathbb{N}$ infinito dá origem a um ponto em um sistema dinâmico em que o conjunto que define sua recorrência está contido em $F S(H)$.

Teorema 5.44. Seja $(X, T)$ um sistema dinâmico com $X$ métrico e seja $x_{0} \in X$ um ponto recorrente de $X$. Então para todo $\delta>0$ temos que o conjunto

$$
R_{\delta}=\left\{n \in \mathbb{N} \mid d\left(T^{n}\left(x_{0}\right), x_{0}\right)<\delta\right\}
$$

é um IP-set.

Reciprocamente, se $H$ é um subconjunto infinito de $\mathbb{N}$, então existe um sistema dinâmico $(X, T)$ e um ponto recorrente $x_{0} \in 0$ tal que $R_{1} \subset F S(H)$.

Demonstração. Para a primeira parte, seja $(X, T) \operatorname{com} X$ métrico e $x_{0} \in X$ recorrente e seja $\delta>0$ dado. Vamos construir um conjunto infinito $H \operatorname{com} F S(H) \subset R_{\delta}$.

Por definição de recorrência, existe $h_{1}>0$ satisfazendo $d\left(T^{h_{1}}\left(x_{0}\right), x_{0}\right)<\delta$. Por continuidade existe $\delta_{2}<\delta$ de modo que para todo $x \in X$, se $d\left(x, x_{0}\right)<\delta_{2}$, então $d\left(T^{h_{1}}(x), x_{0}\right)<\delta$, então defina $h_{2}>h_{1}$ tal que $d\left(T^{h_{2}}\left(x_{0}\right), x_{0}\right)<\delta_{2}$. Assim, temos

$$
d\left(T^{h_{1}+h_{2}}\left(x_{0}\right), x_{0}\right) \leq d\left(T^{h_{1}}\left(T^{h_{2}}\left(x_{0}\right)\right), x_{0}<\delta \quad \text { pois } d\left(T^{h 2}\left(x_{0}\right), x_{0}\right)<\delta_{2} .\right.
$$

Suponha que tenhamos definido $h_{1}<h_{2}<\cdots<h_{k} \operatorname{com} F S\left(\left\{h_{i} \mid 1 \leq i \leq k\right\}\right) \subset R_{\delta}$. Defina $\delta_{k+1}<\delta$ de modo que para todo $x \in X$ e $m \in F S\left(\left\{h_{i} \mid 1 \leq i \leq k\right\}\right)$, se $d\left(x, x_{0}\right)<\delta_{k+1}$ então $d\left(T^{m}(x), x_{0}\right)<\delta$ (encontrando um $\delta_{m}$ apropriado para cada $m$ e tomando o mínimo). Defina $h_{k+1}>h_{k}$ tal que $d\left(T^{h_{k+1}}\left(x_{0}\right), x_{0}\right)<\delta_{k+1}$, dessa forma, para todo $m \in F S\left(\left\{h_{i} \mid 1 \leq i \leq k\right\}\right)$, temos

$$
d\left(T^{m+h_{k+1}}\left(x_{0}\right), x_{0}\right) \leq d\left(T^{m}\left(T^{h_{k+1}}\left(x_{0}\right)\right), x_{0}<\delta \quad \text { pois } d\left(T^{h_{k+1}}\left(x_{0}\right), x_{0}\right)<\delta_{k+1} .\right.
$$

Construímos então $H=\left\{h_{n} \mid n \in \mathbb{N}\right\}$ e por construção $F S(H) \subset R_{\delta}$.

Reciprocamente, seja $H \subset \mathbb{N}$ infinito. Considere $X=\{0,1\}^{\mathbb{N}}$ com a métrica usual

$$
d(x, y)=\min \left\{\frac{1}{k} \mid x(i)=y(i) \text { para todo } i<k\right\} \text { e } d(x, y)=1 \text { se } x(1) \neq y(1) .
$$

Defina também $T: X \rightarrow X$ como o shift map $T(x)(n)=x(n+1)$ e $x_{0}=\chi(F S(H))$, a função característica de $F S(H)$.

Sem perda de generalidade vamos supor $H=\left\{h_{n} \mid n \in \mathbb{N}\right\} \operatorname{com} h_{k+1}>\sum_{i=1}^{k} h_{i}$ para todo $k \in \mathbb{N}$, já que podemos encontrar um subconjunto do $H$ original com essa propriedade. Com isso temos

$$
h_{n_{1}}+h_{n_{2}}+\cdots+h_{n_{t}}=h_{m_{1}}+h_{m_{2}}+\ldots, h_{m_{s}} \text { se, e só se, a soma é igual termo a termo. }
$$


Seja $\left\{m_{1}, \ldots, m_{k}\right\} \subset H$ finito e $m=\sum_{i=1}^{k} m_{i}$, então para todo $n<m_{1}$, temos

$$
m_{1}+\cdots+m_{k}+n \in F S(H) \Leftrightarrow n \in F S(H) \text { pela propriedade acima. }
$$

Ou seja, $x_{0}$ e $T^{m}\left(x_{0}\right)$ coincidem ao menos até o termo $m_{1}$, portanto $d\left(T^{m}\left(x_{0}\right), x_{0}\right) \leq \frac{1}{m_{1}}$, o que nos diz que $x_{0}$ é recorrente.

Além disso, por construção

$$
d\left(T^{n}\left(x_{0}\right), x_{0}\right)<1 \Leftrightarrow T^{n}\left(x_{0}\right)(0)=1 \Leftrightarrow x_{0}(n)=1 \Leftrightarrow n \in F S(H) .
$$

Portanto $R_{1}=F S(H)$.

\subsection{Teorema de Gallai}

Para finalizar este capítulo faremos o caminho inverso, provaremos um teorema de recorrência em sistemas dinâmicos e a partir dele concluiremos sua versão em Teoria de Ramsey. $\mathrm{O}$ Teorema de Gallai é também conhecido como o Teorema de Van der Waerden Multidimensional, o que ficará claro com o enunciado.

Teorema 5.45 (Gallai). Sejam $r, d \in \mathbb{N}$. Para toda partição finita $\mathbb{N}^{d}=\bigcup_{i=1}^{r} A_{i}$, existe $j \in$ $\{1, \ldots, r\}$ tal que para quaisquer $k, l \in \mathbb{N}$ existem progressões aritméticas $P_{1}, P_{2}, \ldots, P_{d} \subset \mathbb{N}$ de comprimento $k$ e razão l tal que $P_{1} \times P_{2} \times \cdots \times P_{d} \subset A_{j}$.

Antes de passarmos à versão dinâmica, daremos uma roupagem geométrica a este teorema, o que nos permitirá simplificar bastante o enunciado e também sua demonstração.

Seja $A$ um conjunto de $\mathbb{N}^{d}$, dizemos que um conjunto $B$ é uma imagem afim de $A$ se existem $l \in \mathbb{N}$ e $x \in \mathbb{N}^{d}$ tal que $B=x+l A$, em outras palavras, se podemos transformar o conjunto $A$ no conjunto $B$ através de uma dilatação e uma translação. $O$ seguinte resultado nos garante que o conjunto de imagens afins de qualquer subconjunto finito de $\mathbb{N}^{d}$ é fracamente regular por partições.

Teorema 5.46. Sejam $r, d \in \mathbb{N}$. Para toda partição finita $\mathbb{N}^{d}=\bigcup_{i=1}^{r} A_{i}$, existe $j \in\{1, \ldots, r\}$ tal que para todo conjunto $F \subset \mathbb{N}^{d}$ finito, existem $x \in \mathbb{N}^{d}$ e $l \in \mathbb{N}$ de modo que $x+l F \subset A_{j}$.

O próximo enunciado é, à primeira vista, bastante mais fraco do que o anterior, mas os resultados são, de fato, equivalentes. Definimos $\overrightarrow{0}=(0,0, \ldots, 0)$ e para cada $i \in\{1, \ldots, d\}$, o elemento $e_{i}=(0,0, \ldots, 0,1,0, \ldots, 0)$, ou seja, possuindo 1 na $i$-ésima coordenada e 0 no restante. Denotaremos o conjunto $\left\{\overrightarrow{0}, e_{1}, \ldots, e_{d}\right\}$ por $E$.

Teorema 5.47. Sejam $r, d \in \mathbb{N}$. Para toda partição finita $\mathbb{N}^{d}=\bigcup_{i=1}^{r} A_{i}$, existe $j \in\{1, \ldots, r\}$ contendo uma imagem afim do conjunto E. 
Conseguimos trocar a condição imposta sobre todos os conjuntos finitos de $\mathbb{N}^{d}$ por uma condição sobre um único conjunto, resta ainda mostrar que esses resultados são, de fato, equivalentes.

Teorema 5.48. Os Teoremas 5.45, 5.46 e 5.47 são equivalentes.

Demonstração. Para esta demonstração, denotaremos os Teoremas 5.45, 5.46 e 5.47 por G1, G2 e G3, respectivamente. Comecemos por G1 implica G2.

Seja $\mathbb{N}^{d}=\bigcup_{i=1}^{r} A_{i}$. Seja $F \subset \mathbb{N}^{d}$ finito, então existe $M \in \mathbb{N}$ de modo que $F \subset C=\left\{\left(x_{1}, \ldots, x_{d}\right) \mid x_{i} \leq\right.$ $M, 1 \leq i \leq d\}$. Seja $A_{j}$ o conjunto dado por G1, então existem progressões aritméticas $P_{1}, \ldots, P_{d}$ de comprimento $M$ e razão comum $l$ de modo que $P_{1} \times \cdots \times P_{d} \subset A_{j}$. Defina

$$
x=\left(\min \left(P_{1}\right), \ldots, \min \left(P_{d}\right)\right), \text { então } x+l F \subset x+l C \subset P_{1} \times \cdots \times P_{d} .
$$

Para G2 implica G1, seja $A_{j}$ o conjunto dado por G2. Sejam $k \in \mathbb{N}$ dado e defina $C_{k}=$ $\left\{\left(y_{1}, \ldots, y_{d}\right) \mid x_{i} \leq k, 1 \leq i \leq d\right\}$. Por $\mathrm{G} 2$ existem $x=\left(x_{1}, \ldots, x_{d}\right) \in \mathbb{N}^{d}$ e $l \in \mathbb{N}$ de modo que $x+l C_{k} \subset A_{j}$, mas $C_{k}=P_{1} \times \cdots \times P_{d}$ em que $P_{i}=\left\{x_{i}, x_{i}+l, \ldots, x_{i}+k l\right\}$ para $1 \leq i \leq d$, ou seja, são progressões aritméticas de comprimento $k$ e razão comum $l$.

Que G2 implica G3 é imediato, passemos então à última implicação.

Seja $\mathbb{N}^{d}=\bigcup_{i=1}^{r} A_{i}$ uma partição finita de $\mathbb{N}^{d}$ e seja $F=\left\{f_{1}, \ldots, f_{t}\right\} \subset \mathbb{N}^{d}$ finito. Vamos definir uma partição $B_{1}, \ldots, B_{r}$ de $\mathbb{N}^{t}$ da seguinte forma: um ponto $y=\left(y_{1}, \ldots, y_{t}\right) \in B_{i}$ se, e somente se, $y_{1} f_{1}+y_{2} f_{2}+\cdots+y_{t} f_{t} \in A_{i}$. Por G3 temos que existem $j \in\{1, \ldots, r\}, l \in \mathbb{N}$ e $z \in \mathbb{N}^{d}$ tal que $z+l\left\{\overrightarrow{0}, e_{1}, \ldots, e_{t}\right\} \subset B_{j}$. Vejamos o que isso significa:

$$
\begin{array}{cccc}
\left(z_{1}+l, z_{2}, \ldots, z_{t}\right) \in B_{j} & \Leftrightarrow & {\left[z_{1} f_{1}+\cdots+z_{t} f_{t}\right]+l f_{1} \in A_{j}} \\
\left(z_{1}, z_{2}+l, \ldots, z_{t}\right) \in B_{j} & \Leftrightarrow & {\left[z_{1} f_{1}+\cdots+z_{t} f_{t}\right]+l f_{2} \in A_{j}} \\
\vdots & \vdots & \vdots \\
\left(z_{1}, z_{2}, \ldots, z_{t}+l\right) \in B_{j} & \Leftrightarrow & {\left[z_{1} f_{1}+\cdots+z_{t} f_{t}\right]+l f_{t} \in A_{j}}
\end{array}
$$

Defina $x=z_{1} f_{1}+\cdots+z_{t} f_{t}$ e note que $x+l F \subset A_{j}$.

A seguir consideraremos não apenas uma função, mas diversas funções sobre um mesmo espaço. Neste caso um conjunto é dito invariante se é invariante com relação a todas as funções simultaneamente.

Proposição 5.49. Se $X$ é espaço métrico compacto e $T_{1}, \ldots, T_{\ell}: X \rightarrow X$ são homeomorfismos que comutam entre si, então existe um conjunto $Y \subset X$ fechado invariante não vazio minimal.

Demonstração. Considere o conjunto $\mathscr{F}=\{F \subset X \mid F$ é fechado, invariante e não vazio $\}$ com a ordem da inclusão (é claro que $\mathscr{F} \neq \emptyset$, pois $X \in \mathscr{F}$ ). Seja $\mathscr{C} \subset \mathscr{F}$ uma cadeia nesta ordem, então $\mathscr{C}$ é formado por conjuntos encaixados, e como $X$ é compacto, temos que $\bigcap_{A \in \mathscr{C}} A$ é um fechado não vazio, além disso, se $x \in \bigcap_{A \in \mathscr{C}} A$, então $T i(x) \in \bigcap_{A \in \mathscr{C}} A$ para $i \in\{1, \ldots, \ell\}$, pois cada $A \in \mathscr{C}$ é invariante. Mostramos então que $\mathscr{F}$ é um conjunto parcialmente ordenado em que 
toda cadeia ascendente admite minorante, estamos então nos termos do Lema de Zorn, que diz que esta ordem admite um elemento minimal $Y$.

É fácil ver que para $1 \leq i \leq d, T_{i} \Upsilon_{Y}$ é homeomorfismo de $Y$ em $Y$. Para os dois próximos resultados consideraremos que $X$ não admite espaços invariantes fechados próprios, pois poderemos restringir nossa análise a um subespaço $Y$ com esta propriedade.

Lema 5.50. Sejam $X$ espaço compacto invariante minimal com relação aos homeomorfismos $T_{1}, \ldots, T_{\ell}: X \rightarrow X$ e $V \subset X$ aberto não vazio. Seja $G$ o grupo dos homeomorfismos gerados por $T_{1}, \ldots, T_{\ell}$, então $X=\bigcup_{S \in G} S^{-1}(V)$.

Demonstração. Considere $Y=\bigcup_{S \in G} S^{-1}(V)$. Note que $Y$ é aberto, portanto $X \backslash Y$ é fechado. Afirmamos que $X \backslash Y$ é invariante. Suponha o contrário, então existem $x \in X \backslash Y$ e $S \in G$ com $S^{-1}(x) \in Y$. Tome $R \in G$ de modo que $S^{-1}(x) \in R^{-1}(V)$ e defina $B=S \circ R^{-1}(V)$. Note que $x \in B$ e $R \circ S^{-1} \in G$, mas $\left(R \circ S^{-1}\right)^{-1}(V)=S \circ R^{-1}(V)=B$, então $B \subset Y$, contradizendo a escolha de $x$.

Como $X$ é minimal, temos que $X \backslash Y=X$ ou $X \backslash Y=\emptyset$. Como $Y$ é não vazio, temos que a segunda possibilidade.

Lema 5.51. Seja $X$ espaço métrico compacto e $T_{1}, \ldots, T_{\ell}$ homeomorfismos de $X$ em $X$, então para todo $\varepsilon>0$ existe $\delta>0$ tal que para todo $x, y \in X$ temos que se $d(x, y)<\delta$, então $d\left(T_{i}(x), T_{i}(y)\right)<$ $\varepsilon, 1 \leq i \leq \ell$.

Demonstração. Como $X$ é compacto, cada $T_{i}$ é uniformemente contínua, portanto existe $\delta_{i}>0$ que satisfaz a condição acima somente para $T_{i}$. Tome $\delta=\min \left\{\delta_{i} \mid 1 \leq i \leq \ell\right\}$.

Teorema 5.52. Seja $\left(X, T_{1}, \ldots, T_{\ell}\right)$. Para todo $\ell \in \mathbb{N}$, temos:

1. Para todo $\varepsilon>0$, existe $x \in X$ e $n \in \mathbb{N}$ tal que $d\left(x, T_{i}^{n}(x)\right)<\varepsilon$ para $i \in\{1 \ldots, \ell\}$.

2. Para todo $\varepsilon>0$, se $X$ é minimal e invariante, então para todo $U \subset X$ aberto existe $n \in \mathbb{N}$ tal que $U \cap T_{1}^{-n} \cap \cdots \cap T_{\ell}^{-n} \neq \emptyset$.

Demonstração. Denote por $\mathscr{A}_{k}$ a afirmação (1) para o caso $\ell=k$ e $\mathscr{B}_{k}$ a afirmação (2) para o caso $\ell=k$. Por indução demonstraremos que $\mathscr{A}_{k} \Rightarrow \mathscr{B}_{k}$ e $\mathscr{B}_{k} \Rightarrow \mathscr{A}_{k+1}$.

Note que $\mathscr{A}_{1}$ nada mais é do que a versão dinâmica do Teorema de Van der Waerden.

Vamos mostrar a primeira implicação: Suponha $\mathscr{A}_{k}$ e seja $\left(X, T_{1}, \ldots, T_{k+1}\right)$ como no enunciado de (2) e $U \subset X$ aberto não vazio. Tome $u \in U$ e $\varepsilon>0 \operatorname{com} B_{\varepsilon}(u) \subset U$ e defina $V=B_{\frac{\varepsilon}{2}}(u)$. Defina também $G$ como o grupo de homeomorfismos gerados por $T_{1}, \ldots, T_{k+1}$.

Pelo Lema 5.50 e por compacidade, existem $S_{1}, \ldots, S_{r} \in G$ tais que $\bigcup_{i=1}^{r} S_{i}^{-1}(V)=X$.

Seja $\delta$ como no Lema 5.51 para o parâmetro $\frac{\varepsilon}{2}$. Por $\mathscr{A}_{k}$, existe $y \in X$ e $n \in \mathbb{N}$ de modo que 
$d\left(y, T_{i}^{n}(y)\right)<\delta, 1 \leq i \leq k$. Seja $j$ tal que $y \in S_{j}^{-1}(V)$ e defina $x=S_{j}(y)$, então $x \in V$. Mas para todo $i \in\{1, \ldots, k\}$,

$$
d\left(x, T_{i}^{n}(x)\right)=d\left(S_{j}(y), T_{i}^{n}\left(S_{j}(y)\right)\right)=d\left(S_{j}(y), S_{j}\left(T_{i}^{n}(y)\right)\right)<\frac{\varepsilon}{2} .
$$

Assim, $d\left(x, T_{i}^{n}(x)\right)<\frac{\varepsilon}{2}$ e $x \in V, \operatorname{logo} d\left(V, T_{i}^{n}(x)\right)<\frac{\varepsilon}{2}$ e, consequentemente, $T_{i}^{n}(x) \in U$.

Portanto, temos que $\left\{x, T_{1}^{n}(x), \ldots, T_{k}^{n}(x)\right\} \subset U$, ou seja, $x \in U \cap T_{1}^{-n}(U) \cap \cdots \cap T_{k}^{-n}(U)$, concluindo a demonstração de $\mathscr{A}_{k} \Rightarrow \mathscr{B}_{k}$.

Para a segunda implicação mostraremos o caso $k=2$, ou seja, $\mathscr{B}_{2} \Rightarrow \mathscr{A}_{3}$, sendo a generalização imediata.

Seja $\left(X, T_{1}, T_{2}, T_{3}\right)$ e suponha que $X$ seja fechado invariante minimal. Seja $U_{0} \subset X$ aberto não vazio de diâmetro menor que $\frac{\varepsilon}{2}$. Defina $V_{0}:=U_{0} \cap\left[T_{1} T_{3}^{-1}\right]^{-n_{1}}\left(U_{0}\right) \cap\left[T_{2} T_{3}^{-1}\right]^{-n_{1}}\left(U_{0}\right)$, então, por $\mathscr{B}_{2}$, existe $n_{1} \in \mathbb{N}$ tal que $V_{0} \neq \emptyset$. Seja agora $U_{1}$ aberto não vazio de diâmetro menor que $\frac{\varepsilon}{2}$ com

$$
U_{1} \subset T_{3}^{-n_{1}}\left(V_{0}\right)=T_{1}^{-n_{1}}\left(U_{0}\right) \cap T_{2}^{-n_{1}}\left(U_{0}\right) \cap T_{3}^{-n_{1}}\left(U_{0}\right) .
$$

Suponha que já existam $U_{0}, \ldots, U_{r}$ abertos não vazios de diâmetro menor que $\frac{\varepsilon}{2}$ e $n_{1}, \ldots, n_{r}$ tais que

$U_{j} \subset T_{1}^{-\left(n_{i+1}+n_{i+2}+\cdots+n_{j}\right)}\left(U_{i}\right) \cap T_{2}^{-\left(n_{i+1}+n_{i+2}+\cdots+n_{j}\right)}\left(U_{i}\right) \cap T_{3}^{-\left(n_{i+1}+n_{i+2}+\cdots+n_{j}\right)}\left(U_{i}\right), 1 \leq i \leq j \leq r$.

Por $\mathscr{B}_{2}$ existe $n_{r+1}$ de modo que $U_{r} \cap\left[T_{1} T_{3}^{-1}\right]^{-n_{r+}}\left(U_{r}\right) \cap\left[T_{2} T_{3}^{-1}\right]^{-n_{r+1}}(U-r) \neq \emptyset$, portanto existe um aberto não vazio $U_{r+1}$ de diâmetro menor que $\frac{\varepsilon}{2}$ tal que

$$
U_{r+1} \subset T_{1}^{-n_{r+1}}\left(U_{r}\right) \cap T_{2}^{-n_{r+1}}\left(U_{r}\right) \cap T_{3}^{-n_{r+1}}\left(U_{r}\right) .
$$

Usando isto e a hipótese de indução temos que, para todo $1 \leq i \leq r+1$,

$$
U_{r+1} \subset T_{1}^{-\left(n_{i+1}+n_{i+2}+\cdots+n_{r+1}\right.}\left(U_{i}\right) \cap T_{2}^{-\left(n_{i+1}+n_{i+2}+\cdots+n_{r+1}\right.}\left(U_{i}\right) \cap T_{3}^{-\left(n_{i+1}+n_{i+2}+\cdots+n_{r+1}\right.}\left(U_{i}\right) .
$$

Portanto existem duas sequências $\left(U_{n}\right)_{n \in \mathbb{N}}$ de abertos não vazios e $\left(n_{j}\right)_{j \in \mathbb{N}}$ com tal propriedade. Defina também uma sequência $\left(x_{n}\right)_{n \in \mathbb{N}}$ de modo que $\forall n \in \mathbb{N}, x_{n} \in U_{n}$, então pela compacidade de $X$ podemos encontrar $i, j \in \mathbb{N}$ de modo que $d\left(x_{i}, x_{j}\right)<\frac{\varepsilon}{2}$ e $i<j$.

Usando a propriedade dos $U_{n}$ que construímos, definindo $n=n_{i+1}+\cdots+n_{j}$, temos que $\left.T_{1}^{n}\left(x_{j}\right), T_{2}^{n}\left(x_{j}\right), T_{3}^{n}\left(x_{j}\right)\right\} \subset U_{i}$, portanto $d\left(x_{i}, T_{m}^{n}\left(x_{j}\right)\right)<\frac{\varepsilon}{2}, m=1,2,3$. Finalmente, para $m=$ $1,2,3$, temos $d\left(x_{j}, T_{m}^{n}\left(x_{j}\right)\right) \leq\left(d\left(x_{j}, x_{i}\right)+\left(d\left(x_{i}, T_{m}^{n}\left(x_{j}\right)\right)<\frac{\varepsilon}{2}+\frac{\varepsilon}{2}=\varepsilon\right.\right.$.

Resta mostrar que este último teorema e o Teorema 5.47 são equivalentes. A demonstração é semelhante à feita no caso do Teorema de Van der Waerden.

Teorema 5.53. Os Teoremas 5.52 e 5.47 são equivalentes.

Demonstração. Suponha válido o Teorema 5.52.

Seja $\mathbb{N}^{d}=\bigcup_{i=1}^{r} A_{i}$ uma partição finita de $\mathbb{N}^{d}$ e defina $\Omega=\{1, \ldots, r\}^{\mathbb{N}^{d}}$, ou seja, o espaço de 
todas as partições de $\mathbb{N}^{d}$. Note que $\Omega$ é compacto (Teorema de Tychonoff) e, além disso, admite a seguinte métrica:

$$
d(\alpha, \beta)=\inf \left\{\frac{1}{k} \mid \alpha\left(n_{1}, \ldots, n_{d}\right)=\beta\left(n_{1}, \ldots, n_{d}\right), \text { para todo } 1 \leq n_{1}, n_{2} \ldots, n_{d} \leq k\right\} .
$$

Definimos o ponto $\gamma \in \Omega$ como $\gamma\left(n_{1}, \ldots, n_{d}\right)=i \Leftrightarrow\left(n_{1}, \ldots, n_{d}\right) \in A_{i}$. Finalmente definimos o espaço métrico compacto $X=\overline{\left\{T_{i}^{k}(\gamma) \mid k \in \mathbb{N}, i \in\{1 \ldots, r\}\right\}}$ e para cada $i \in\{1, \ldots, d\}$ as funções $T_{i}(\alpha)\left(n_{1}, \ldots, n_{i}, \ldots, n_{d}\right)=\alpha\left(n_{1}, \ldots, n_{i}+1, \ldots, n_{d}\right)$, que são claramente contínuas e comutam entre si.

Pelo Teorema 5.52, existe $\xi \in X$ e $l \in \mathbb{N}$ tal que $d\left(\xi, T_{i}^{l}(\xi)\right)<1$ para $i \in\{1, \ldots, r\}$, o que significa que $\xi(\overrightarrow{0})=\xi\left(l e_{1}\right)=\xi\left(l e_{2}\right)=\cdots=\xi\left(l e_{d}\right)$. Como $\xi \in X$, existem $x_{1}, \ldots, x_{d} \in \mathbb{N}$ tal que $d\left(\xi, T_{1}^{x_{1}} \circ T_{2}^{x_{2}} \circ \cdots \circ T_{d}^{x_{d}}(\gamma)\right)<l$, temos então

$$
\alpha\left(x_{1}, \ldots, x_{d}\right)=\alpha\left(x_{1}+l, x_{2}, \ldots, x_{d}\right)=\cdots=\alpha\left(x_{1}, x_{2}, \ldots, x_{d}+l\right)=\xi(0) .
$$

Portanto se $x=\left(x_{1}, \ldots, x_{d}\right)$, temos que $x+l E \subset A_{\xi(0)}$.

Reciprocamente, seja $X$ um espaço métrico compacto, $T_{1}, \ldots, T_{d}$ funções contínuas que comutam entre si e $\varepsilon>0$. Tome a cobertura $\mathscr{C}^{\prime}=\left\{B_{\varepsilon}(x) \mid x \in X\right\}$. Por $X$ ser compacto existe uma subcobertura finita $\mathscr{C}=\left\{B_{1}, \ldots, B_{r}\right\}$. Fixe $y \in X$ e defina a partição finita $\mathbb{N}^{d}=\bigcup_{i=1}^{r} A_{i}$ como $\left(n_{1}, \ldots, n_{d}\right) \in A_{i}$ se, e somente se, $T_{1}^{n_{1}} \circ \cdots \circ T_{d}^{n_{d}}(y) \in B_{i}$. Mas então o Teorema 5.47 nos garante que existem $j \in\{1, \ldots, r\}, a \in \mathbb{N}^{d}$ e $l \in \mathbb{N}$ tais que $a+l E \subset A_{j}$. Portanto para $x=T_{1}^{a_{1}} \circ \cdots \circ$ $T_{d}^{a_{d}}(y)$, temos que $T_{i}^{l}(x) \in B_{j}$ para $i \in\{1, \ldots, r\}$.

Na seção anterior discutimos que os resultados da Teoria de Ramsey podem ser traduzidos para resultados de recorrência. Da mesma forma que fizemos isso para o Teorema de Van der Waerden em 5.43, podemos provar que o Teorema 5.45 é equivalente ao seguinte resultado, apenas substituindo a definição do sistema dinâmico $(\Omega, T)$ pela usada no teorema anterior.

Teorema 5.54. Seja $\left(X, T_{1}, \ldots, T_{d}\right)$ um sistema dinâmico em que $X$ é espaço métrico compacto e $T_{i}$ é contínua para cada $i \in\{1, \ldots, d\}$ e comutam entre si. Existe um ponto $x \in X$ tal que para todo $\varepsilon>0$ e todo $k \in \mathbb{N}$, existe $n \in \mathbb{N}$ tal que $d\left(x, T_{i}^{j n}(x)\right)<\varepsilon, 1 \leq j<k, 1 \leq i \leq d$.

\subsection{Alguns problemas em aberto}

Na Seção 5.4 vimos que podemos conseguir o Teorema de Hindman simultaneamente para a estrutura aditiva e multiplicativa de $\mathbb{N}$, garantindo a existência de dois conjuntos infinitos distintos, um cujas somas finitas e outro cujos produtos finitos sejam monocromáticos. Naturalmente nos perguntamos se esse resultado é válido considerando as somas e produtos finitos de um mesmo conjunto infinito, mas em Hindman e Strauss (2012), Teorema 17.16, é demonstrado a negativa.

No Teorema de Van der Waerden, não podemos garantir a existência de progressões aritméticas 
infinitas, mas apenas de progressões finitas arbitrariamente longas, o que nos inspira a seguinte pergunta:

Questão 5.55. Dada qualquer partição finita de $\mathbb{N}$ e qualquer $k \in \mathbb{N}$, existe um conjunto $H \subset \mathbb{N}$ com $|H|=k$ de modo que o conjunto $F S(H) \cup F P(H)$ esteja contido em um mesmo elemento da partição?

Neil Hindman, em Bergelson e Rothschild (2009), diz estar completamente convencido de que a resposta é afirmativa, mas ainda não se sabe nem mesmo se o problema análogo referente ao Teorema de Schur é verdadeiro.

Questão 5.56. Dada qualquer partição finita de $\mathbb{N}$, existem elementos $x, y \in \mathbb{N}$ distintos tal que o conjunto $\{x, y, x+y, x \cdot y\}$ esteja contido em um mesmo elemento da partição?

Não se sabe a resposta nem mesmo se exigirmos apenas que $x+y$ e $x \cdot y$ estejam na mesma parte.

Ainda relacionado ao Teorema de Hindman, como fizemos sua demonstração via ultrafiltros, temos uma consequência imediata ligeiramente mais forte:

Teorema 5.57. Seja $D \subset \mathbb{N}$. Se existir $K \subset D$ infinito com $F S(K) \subset D$, então para toda partição finita $\bigcup_{i=1}^{r} A_{i}=D$ existe $j \in\{1, \ldots, r\}$ e $H \subset D$ infinito tal que $F S(H) \subset A_{j}$.

Dado um conjunto infinito $H \subset \mathbb{N}$ e $k \in \mathbb{N}$, considere o conjunto

$$
F S_{k}(H)=\left\{\sum_{n \in F} n \mid F \subset H \text { e }|F| \leq k\right\}
$$

É claro que $F S_{k}(H) \subset F S(H)$, pois consideramos apenas somas de até $k$ elementos distintos de $H$, portanto resultados envolvendo as somas finitas imediatamente se estendem a esses casos. Curiosamente, como apresentado em Hindman, Leader e Strauss (2003), não se sabe se é possível enfraquecer a hipótese do resultado acima.

Questão 5.58. Existem $D \subset \mathbb{N}$ e $k \in \mathbb{N}$ tal que para toda partição finita $\bigcup_{i=1}^{r} A_{i}=D$ de $D$ existe $j \in\{1, \ldots, r\}$ e $H \subset D$ infinito tal que $F S_{k}(H) \subset A_{j}$, mas que não contenha um conjunto infinito $K \subset D \operatorname{com} F S(K) \subset D$ ?

De fato, ainda não se conhece uma demonstração para o fato de que para toda partição finita $\bigcup_{i=1}^{r} A_{i}=\mathbb{N}$ de $\mathbb{N}$ existe $j \in\{1, \ldots, r\}$ e $H \subset D$ infinito tal que $F S_{2}(H) \subset A_{j}$ sem que ela demonstre o Teorema de Hindman.

Questão 5.59. Existe algum conjunto $D \subset \mathbb{N}$ de modo que para toda partição finita $\bigcup_{i=1}^{r} A_{i}=D$ de $D$, existe um conjunto infinito $H \subset(\mathbb{N} \backslash D)$ e $j \in\{1, \ldots, r\} \operatorname{com} F S(H) \backslash H \subset A_{j}$ ? 
O primeiro motivo para que esta seja uma questão de interesse é que o resultado é válido para $H$ finito. o segundo é que caso a resposta seja negativa, então teríamos também uma resposta negativa para o problema da existência de homomorfismos contínuos entre $\beta \mathbb{N}$ e $\mathbb{N}^{*}$ (Questão 3.28), como demonstrado em Davenport, Hindman e Leader (2000), Corolário 3.5.

Por fim, mesmo não tendo sido tratado neste trabalho, a versão finita dos teoremas apresentados ainda tem possibilidade de muitos avanços. O Teorema de Van der Waerden em sua versão finita, por exemplo, diz que para todos $k, r \in \mathbb{N}$, existe $N \in \mathbb{N}$ tal que para toda partição $\bigcup_{i=1}^{r} A_{i}=$ $\{1, \ldots, N\}$, existe uma progressão aritmética de $k$ termos contida em um mesmo elemento da partição, denotamos por $W(r, k)$ o menor de tais $N \in \mathbb{N}$.

O problema óbvio é descobrir os valores de $W(k, r)$ para cada $k, r \in \mathbb{N}$, mas como é de se esperar, esse problema é bastante complexo. Até o momento são conhecidos apenas 7 desse valores:

- $W(2,3)=9, W(2,4)=35, W(2,5)=178, W(2,6)=1132$

- $W(3,3)=27, W(3,4)=293$

- $W(4,3)=76$

sendo o resultado mais recente de 2012, por Michal Kouril, que computou o valor de $W(3,4)$ em Kouril (2012).

Existem limitantes para diversas outras duplas, mas o melhor limitante superior conhecido para qualquer $k, r \in \mathbb{N}$ foi dado por William Gowers, em 2001 (Gowers (2001), Teorema 18.2), com o impressionante

$$
W(k, r) \leq 2^{2^{2^{2^{2^{k+9}}}}}
$$

Um problema relacionado aparentemente muito simples é: defina $w(s, t)$ como o menor número $N \in \mathbb{N}$ de modo que para toda partição $A \cup B=\{1, \ldots, N\}$, existe uma progressão aritmética de comprimento $s$ contida em $A$ ou uma progressão de comprimento $t$ contida em $B$, então é verdade que $w(s, t) \geq w(s+1, t-1)$ para todo $s \geq t \geq 2$ ?

Problemas análogos também existem para as versões finitas dos Teoremas de Hindman, Ramsey e Hales-Jewett. 

A ideia de se buscar teoremas envolvendo a densidade natural começa em 1936, quando Erdős e Turán conjecturaram que qualquer conjunto com densidade natural não nula conteria progressões aritméticas de comprimento arbitrário. Em 1975, com um argumento complexo e puramente combinatório, Szemerédi demonstrou que a conjectura é verdadeira.

Pouco depois, em 1977, no artigo Furstenberg (1977), Furstenberg desenvolveu uma demonstração alternativa, via Teoria Ergódica, que rendeu vários frutos em Teoria dos Números, incluindo generalizações do Teorema de Szemerédi e resultados de densidade envolvendo os Teoremas de Hindman e Hales-Jewett. Ele desenvolve a teoria de recorrência em sistemas dinâmicos, com diversas aplicações à Teoria de Ramsey e Teoria dos Números, em Furstenberg (1981). Um bom texto, com um panorama sobre a área, é Bergelson (1996).

Apresentaremos neste capítulo a abordagem de Neil Hindman e Dona Strauss em Hindman e Strauss (2012), com aplicações presentes nos artigos Bergelson (1986) e Hindman e Bergelson (1988).

\subsection{Densidade de Banach}

O conjunto $\mathbb{N}$ admite uma forma natural de se determinar o "tamanho" de um conjunto através da densidade natural. Intuitivamente, pensamos que o conjunto dos números pares são "maiores" do que o conjunto dos números múltiplos de 4 , mas essa ideia não se expressa pela noção de cardinalidade. A ideia é captar quão "dispersos" são os elementos do conjunto, o que nos leva a definição da densidade natural $d: \mathscr{P}(\mathbb{N}) \rightarrow[0,1]$ dada por

$$
d(A)=\lim _{n \rightarrow \infty} \frac{|A \cap[1, n]|}{n} .
$$

Analogamente à notação dos números reais, usaremos a notação de intervalos $[m, n]$ para o conjunto $\{m, m+1, \ldots, n\}$ e seus análogos para $(m, n),(m, n]$ e $[m, n)$.

$\mathrm{O}$ que esta definição faz é determinar a fração de $\mathbb{N}$ que o conjunto $A$ ocupa, porém este 
limite pode não existir. Para contornar este problema podemos usar a densidade superior $\bar{d}: \mathscr{P}(\mathbb{N}) \rightarrow[0,1]$ ou a densidade inferior $\underline{d}: \mathscr{P}(\mathbb{N}) \rightarrow[0,1]$, dadas respectivamente por

$$
\bar{d}(A)=\lim _{n \rightarrow \infty} \sup \frac{|A \cap[1, n]|}{n} \quad \text { e } \quad \underline{d}(A)=\lim _{n \rightarrow \infty} \inf \frac{|A \cap[1, n]|}{n} .
$$

Note que se $d(A)>0$ ou $\underline{d}(A)>0$, então $\bar{d}(A)>0$, então a densidade superior nos dá a definição mais geral de densidade não nula dentre as três possibilidades. Além disso, os conjuntos não nulos nesse sentido podem ser caracterizados via ultrafiltros.

Teorema 6.1. Sejam $(S,+)$ um semigrupo e $\mathscr{G} \subset \mathscr{P}(S) \backslash\{\emptyset\}$. Defina

$$
\beta \mathscr{G}=\{p \in \beta S \mid \text { para todo } A \in p, A \in \mathscr{G}\}
$$

São equivalentes:

1. Seja $G \subset S . G \in \mathscr{G}$ se, e somente se, existe um ultrafiltro $p \in \beta \mathscr{G}$ com $G \in p$.

2. Sejam $A, B \subset S$. Se $A \subset B$ e $A \in \mathscr{G}$, então $B \in \mathscr{G}$ e se $A \cup B \in \mathscr{G}$, então $A \in \mathscr{G}$ ou $B \in \mathscr{G}$.

Demonstração. Note que o resultado é imediato por vacuidade se $\mathscr{G}=\emptyset$. Vamos então supor $\mathscr{G} \neq \emptyset$.

$1 \Rightarrow 2$ ) Para a primeira parte, se $A \in \mathscr{G}$ e $A \subset B$, existe $p \in \beta \mathscr{G}$ com $A \in p$, portanto $B \in p$, $\log$, $B \in \mathscr{G}$.

Para a segunda parte, se $A \cup B \in \mathscr{G}$, existe $p \in \beta \mathscr{G} \operatorname{com} A \cup B \in p, \log A \in p$ ou $B \in p$, e assim $A \in \mathscr{G}$ ou $B \in \mathscr{G}$.

$2 \Rightarrow 1)$ Em primeiro lugar temos que mostrar que a condição (2) implica $\beta \mathscr{G} \neq \emptyset$.

Seja $G \in \mathscr{G}$ e considere o conjunto $\mathscr{F}_{G}=\{F \subset \mathscr{G} \mid F$ é um filtro e $G \in F\}$. Com a ordem da inclusão, $\mathscr{F}_{G}$ é um conjunto parcialmente ordenado satisfazendo as condições do Lema de Zorn, portanto existe um elemento maximal $p \in \mathscr{F}_{G}$. Vamos mostrar que $p$ é um ultrafiltro.

Seja $A \subset S$ e suponha que $A \notin p$. Note que $A \cup(S \backslash A)=S$, então $A \in \mathscr{G}$ ou $(S \backslash A) \in \mathscr{G}$. Suponha que $(S \backslash A) \notin \mathscr{G}$, neste caso teríamos $(S \backslash A) \notin p$ e $A \in \mathscr{G}$. Assim, para todo $B \in p, B \nsubseteq(S \backslash A)$, $\operatorname{logo}, A \cap B \neq \emptyset$, o que implica $p \cup\{A\}$ ter a PIF, o que contradiz a maximalidade de $p$.

Portanto $(S \backslash A) \in \mathscr{G}$, e como $B \nsubseteq A$ para todo $B \in p, p \cup\{(S \backslash A)\}$ tem a PIF e, por maximalidade de $p,(S \backslash A) \in p$. Concluímos então que $p$ é um ultrafiltro e, consequentemente, que existe para todo $G \in \mathscr{G}$, existe um ultrafiltro $p \in \beta \mathscr{G}$ com $G \in p$. Em particular, $\beta \mathscr{G} \neq \emptyset$.

Para a primeira equivalência em (1), suponha que $G \in \mathscr{G}$, então existe um ultrafiltro $p \in \mathscr{F}_{G}$, $\operatorname{logo} p \in \beta \mathscr{G}$ e $G \in p$.

Reciprocamente, seja $G \subset S$ e suponha que exista $p \in \beta \mathscr{G}$ com $G \in p$, então pela definição de $\beta \mathscr{G}$ temos que $G \in \mathscr{G}$.

Portanto, se mostrarmos que o conjunto $\mathscr{G}=\{A \subset \mathbb{N} \mid \bar{d}(A)>0\}$ satisfaz o item (2) deste último teorema, teremos que $\mathscr{G}$ admite uma caracterização via ultrafiltros. 
Em $\mathbb{N}$, é imediato que se $\bar{d}(A)>0$ e $A \subset B$, então $\bar{d}(B)>0$. Além disso, como para todo $n \in \mathbb{N}$

$$
\frac{|(A \cup B) \cap[1, n]|}{n} \leq \frac{|A \cap[1, n]|}{n}+\frac{|B \cap[1, n]|}{n},
$$

temos que $\bar{d}(A \cup B) \leq \bar{d}(A)+\bar{d}(B)$ e consequentemente $\bar{d}(A \cup B)>0$ implica $\bar{d}(A)>0$ ou $\bar{d}(B)>0$.

Logo, pelo teorema anterior, o conjunto definido a seguir é não vazio.

Definição 6.2. $\mathrm{O}$ conjunto $\Delta \subset \beta \mathbb{N}$ é definido como:

$$
\Delta=\{p \in \beta \mathbb{N} \mid \forall A \in p, \bar{d}(A)>0\} .
$$

O Teorema de Szemerédi, na seção seguinte, pode ser demonstrado com uma formulação de densidade mais geral, em que para cada $n \in \mathbb{N}$ o limite não somente avalia a densidade do conjunto $A$ com relação ao segmento inicial de tamanho $n$, mas com relação a todos os segmentos de tamanho $n$.

Definição 6.3. Seja $A \subset \mathbb{N}$. Definimos a densidade de Banach superior de $A$ como

$$
d^{*}(A)=\lim _{n \rightarrow \infty} \sup _{k \in \mathbb{N}} \frac{\mid A \cap[k, k+n)) \mid}{n} .
$$

Pelas definições de $\bar{d}$ e $d^{*}$ é imediato que $\bar{d} \leq d^{*}$, portanto qualquer conjunto $A \subset \mathbb{N}$ com $\bar{d}(A)>0$, temos que $d^{*}(A)>0$. O exemplo a seguir mostra que a recíproca não vale.

Exemplo 6.4. Vamos construir um conjunto $A \subset \mathbb{N}$ tal que $\bar{d}(A)=0$ e $d^{*}(A)=1$. No primeiro passo defina $1 \in A$ e $2 \notin A$, no segundo passo $4,5 \in A$ e $6,7 \notin A$, no terceiro $8,9,10 \in A$ e $11,12,13,14,15 \notin A$ e assim por diante, ou seja, no $n$-ésimo passo defina que os próximos $n$ números estejam em $A$ e que os outros $2^{n}-n$ não estejam em $A$, como abaixo, em que $\bullet$ denota os elementos de $A$ e $\circ$ os elementos que não pertencem a $A$ :

$$
\begin{array}{llllllllllllllllllllll}
1 & 2 & 3 & 4 & 5 & 6 & 7 & 8 & 9 & 10 & 11 & 12 & 13 & 14 & 15 & 16 & 17 & 18 & 19 & 20 & 21 & 22 \ldots
\end{array}
$$

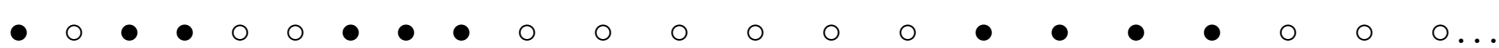

Não é difícil ver que

$$
\bar{d}(A)=\lim _{n \rightarrow \infty} \frac{1+2+\cdots+n}{2+4+\cdots+2^{n}}=0 .
$$

Por outro lado,

$$
d^{*}(A)=\lim _{n \rightarrow \infty} \sup _{k \in \mathbb{N}} \frac{|A \cap(k, k+n)|}{n} \quad \text { e } \quad \sup _{k \in \mathbb{N}} \frac{|A \cap[k, k+n)|}{n}=1 \text { para todo } n,
$$

pois $A$ contém intervalos de comprimento arbitrário.

Assim como para $\bar{d}$, temos que $d^{*}(A)>0$ e $A \subset B$, então $d^{*}(B)>0$ e se $d^{*}(A \cup B)>0$, então $d^{*}(A)>0$ ou $d^{*}(B)>0$, portanto também faz sentido a definição seguinte:

Definição 6.5. O conjunto $\Delta^{*} \subset \beta \mathbb{N}$ é definido como

$$
\Delta^{*}=\left\{p \in \beta \mathbb{N} \mid \forall A \in p, d^{*}(A)>0\right\} .
$$




\subsection{Princípio da Correspondência de Furstenberg e Teo- rema de Szemerédi}

No Teorema 5.43, construímos o espaço de funções $\Omega=\{1, \ldots, r\}^{\mathbb{N}}$ e sobre ele definimos o shift map. Considere a função $\alpha: \mathbb{N} \rightarrow \mathbb{N}$ dada por $\alpha(n)=n+1$. A aplicação do shift map no ponto $f \in \Omega$ nada mais é do que a composição $f \circ \alpha$.

Note que a função $\alpha$ é apenas uma translação em $\mathbb{N}$, portanto é uma função que preserva a densidade dos conjuntos, ou seja $d^{*}(A)=d^{*}\left(\alpha^{-1}(A)\right.$ ) (o mesmo vale para $\bar{d}$ ). Mesmo a densidade não sendo uma medida propriamente dita, ainda nos leva à pergunta se há alguma relação entre a densidade e alguma medida em $\Omega$ preservada pelo shift map.

A partir de agora, ao nos referirmos ao sistema dinâmico $(X, \mathscr{B}, \mu, T)$, queremos dizer que $(X, \mathscr{B}, \mu)$ é um espaço de probabilidade e $T: X \rightarrow X$ preserva medida.

Lema 6.6. Seja $A \subset \mathbb{N} \operatorname{com} d^{*}(A)=\alpha>0$. Então existe uma sequência $\left(I_{n}\right)_{n \in \mathbb{N}}$ de intervalos de $\mathbb{N}$ tal que $\lim _{n \rightarrow \infty}\left|I_{n}\right|=\infty$ e $\lim _{n \rightarrow \infty} \frac{\left|A \cap I_{n}\right|}{\left|I_{n}\right|}=\alpha$.

Demonstração. Para cada $n \in \mathbb{N}$, pela definição de $d^{*}$, existe $m_{n} \geq n$ e $k_{n} \in \mathbb{N}$ de modo que $\frac{\left|A \cap\left[k_{n}, k_{n}+m_{n}\right)\right|}{m_{n}} \geq \alpha-\frac{1}{n}$, então definimos, para cada $n \in \mathbb{N}, I_{n}=\left[k_{n}, k_{n}+m_{n}\right)$. É fácil ver que esta é a sequência procurada.

Nosso intuito é construir uma medida a partir da densidade, então precisamos de algum resultado que nos permita, a partir de uma função finitamente aditiva, construir uma medida. $\mathrm{O}$ lema a seguir é bastante conhecido da Teoria da medida e sua demonstração pode ser encontrada, por exemplo, em Richandson (2009), Seção 2.4.

Lema 6.7 (Teorema da Extensão de Hopf). Seja $X$ um conjunto e $\mathscr{A}$ uma álgebra sobre $X$. Seja $v: \mathscr{A} \rightarrow \mathbb{R} \cup\{\infty\}$ uma função satisfazendo:

1. Para todo $A, B \in \mathscr{A} \operatorname{com} A \cap B=\emptyset, v(A \cup B)=v(A)+v(B)$.

2. Se $\left(A_{n}\right)_{n \in \mathbb{N}} \subset \mathscr{A} \operatorname{com} A_{k+1} \subset A_{k}$ para todo $k \in \mathbb{N}$ e $\bigcap_{n \in \mathbb{N}} A_{n}=\emptyset$, então $\lim _{n \rightarrow \infty} A_{n}=0$.

Então $v$ pode ser estendida a uma medida sobre uma $\sigma$-álgebra $\mathscr{B}$ contendo $\mathscr{A}$.

Teorema 6.8 (Princípio da Correspondência de Furstenberg). Seja $A \subset \mathbb{N}$ com $d^{*}(A)>0$. Existe um sistema dinâmico $(X, \mathscr{B}, \mu, T)$ em que $X$ é um espaço métrico compacto e $T: X \rightarrow X$ é um homeomorfismo, além de um conjunto $A^{\prime} \subset X$ tal que:

1. $\mu\left(A^{\prime}\right)=d^{*}(A)$.

2. Para todo $F \subset \mathbb{N}$ finito, $d^{*}\left(A \cap \bigcap_{n \in F}(-n+A)\right) \geq \mu\left(A^{\prime} \cap \bigcap_{n \in F} T^{-n}\left(A^{\prime}\right)\right)$. 
Demonstração. Seja $\Omega=\{0,1\}^{\mathbb{Z}}$ com a topologia produto e $T: \Omega \rightarrow \Omega$ o shift map, definido por $(T(x))(n)=x(n+1)$, é fácil ver que $T$ é um homeomorfismo. Nestas condições, $\Omega$ é um espaço métrico compacto com a métrica $\rho: \Omega \rightarrow \Omega$ dada por $\rho(x, y)=\frac{1}{k+1}$ onde $k=\min \{|t| \mid t \in$ $\mathbb{Z}$ e $x(t) \neq y(t)\}$. Seja $\xi: \mathbb{Z} \rightarrow\{0,1\} \in \Omega$ dada por:

$$
\xi(z)=\left\{\begin{array}{l}
1 \text { se } z \in A \\
0 \text { se } z \notin A
\end{array}\right.
$$

Ou seja, $\xi$ é a função característica de $A$ visto como subconjunto de $\mathbb{Z}$.

Defina $X=\overline{\left\{T^{n}(\xi) \mid n \in \mathbb{Z}\right\}}$, o fecho da órbita de $\xi$ por $T$. Assim, temos que $X$ é um espaço métrico compacto e $T: X \rightarrow X$ (a restrição de $T$ a $X$ ) é um homeomorfismo, já que $T(X)=X$. Para cada $z \in \mathbb{Z}$, defina $D_{z}=\{x \in X \mid x(z)=1\}$, vamos mostrar que $D_{z}$ é aberto e fechado em $X$. Note que $\{x \in \Omega \mid x(z)=1\}$ é aberto em $\Omega$, portanto $D_{z}=\{x \in \Omega \mid x(z)=1\} \cap X$ é aberto em $X$. Por outro lado, considere para cada $z \in \mathbb{Z}$ a função $\pi_{z}: X \rightarrow\{0,1\}$ dada por $\pi_{z}(x)=x(z)$. É fácil ver que $\pi_{z}^{-1}(i)=\{x \in X \mid x(z)=i\}$ para $i \in\{0,1\}$, portanto $\pi_{z}$ é uma função contínua. Além disso, $D_{z}=\pi_{z}^{-1}(1)$ e $\{1\}$ é fechado em $\{0,1\}$, portanto $D_{z}$ é fechado, demonstrando a afirmação.

Seja $\mathscr{A}$ a álgebra gerada por $\left\{D_{z} \mid z \in \mathbb{Z}\right\}$ e $\mathscr{B}$ a $\sigma$-álgebra gerada por $\mathscr{A}$. Note que $T\left(D_{z}\right)=D_{z-1}$ e $T^{-1}\left(D_{z}\right)=D_{z+1}$, portanto se $B \in \mathscr{B}$, então $T(B) \in \mathscr{B}$ e $T^{-1}(B) \in \mathscr{B}$. Note que a álgebra $\mathscr{A}$ é gerada por conjuntos abertos e fechados tomando uniões e interseções finitas e complementares, portanto todos os elementos de $\mathscr{A}$ são conjuntos abertos e fechados.

Considere a função $\varphi: \mathscr{P}(X) \rightarrow \mathscr{P}(\mathbb{N})$ definida por $\varphi(B)=\left\{n \in \mathbb{N} \mid T^{n}(\xi) \in B\right\}$. Note que $\varphi(B \cup C)=\varphi(B) \cup \varphi(C)$ e $\varphi(B \cap C)=\varphi(B) \cap \varphi(C)$ para todo $B, C \in \mathscr{P}(X)$.

Pelo lema anterior existe uma sequência $\left(I_{n}\right)_{n \in \mathbb{N}}$ de intervalos de $\mathbb{N}$ de modo que $\lim _{n \rightarrow \infty}\left|I_{n}\right|=\infty$ e $\lim _{n \rightarrow \infty} \frac{\left|I_{n} \cap A\right|}{\left|I_{n}\right|}=\alpha$.

Seja $p \in \mathbb{N}^{*}$ e defina a função $v: \mathscr{P}(X) \rightarrow[0,1]$ dada por

$$
v(B)=p-\lim _{n \in \mathbb{N}} \frac{\left|\varphi(B) \cap I_{n}\right|}{\left|I_{n}\right|} .
$$

Temos que $v(X)=1$ e se $B, C \in \mathscr{P}(X)$ forem conjuntos disjuntos, então:

$$
\begin{aligned}
v(B \cup C) & =p-\lim _{n \rightarrow \infty} \frac{\left|\varphi(B \cup C) \cap I_{n}\right|}{\left|I_{n}\right|} \\
& =p-\lim _{n \rightarrow \infty} \frac{\left|\varphi(B) \cap I_{n}\right|+\left|\varphi(C) \cap I_{n}\right|}{\left|I_{n}\right|} \\
& =v(B)+v(C)
\end{aligned}
$$

Portanto $v$ é uma medida finitamente aditiva. Além disso, dado $B \subset X$, temos que $\varphi\left(T^{-1}(B)\right)=$ $(-1+\varphi(B)) \cap \mathbb{N}$, portanto:

$$
v\left(T^{-1}(B)\right)=p-\lim _{n \rightarrow \infty} \frac{\left|(-1+\varphi(B)) \cap I_{n}\right|}{\left|I_{n}\right|}=p-\lim _{n \rightarrow \infty} \frac{\left|\varphi(B) \cap I_{n}\right|}{\left|I_{n}\right|}=v(B)
$$


A segunda igualdade vale pois $\lim _{n \rightarrow \infty} I_{n}=\infty \mathrm{e}\left|(-1+\varphi(B)) \cap I_{n}\right|-\left|\varphi(B) \cap I_{n}\right| \leq 1$, assim $v$ é $T$-invariante em $\mathscr{P}(X)$ e, em particular, em $\mathscr{A}$.

Seja $\left(B_{n}\right)_{n \in \mathbb{N}} \subset \mathscr{A}$ de modo que $B_{k+1} \subset B_{k}$ e $\bigcap_{n \in \mathbb{N}} B_{n}=\emptyset$, como o elementos de $\mathscr{A}$ são fechados e $X$ é compacto, existe $k \in \mathbb{N}$ tal que para todo $j \geq k, B_{j}=\emptyset, \operatorname{logo}_{n} \lim _{n \rightarrow \infty} v\left(B_{n}\right)=0$.

Dessa forma, $v$ está nos termos do Teorema da Extensão de Hopf (Lema 6.7), então podemos definir a extensão $\mu: \mathscr{B} \rightarrow[0,1]$, além disso, para cada $B \in \mathscr{B}$, temos

$$
\mu(B)=\inf \left\{\sum_{C \in \mathscr{G}} v(C)|\mathscr{G} \subset \mathscr{A},| \mathscr{G} \mid \leq \aleph_{0}, \text { e } B \subset \bigcup \mathscr{G}\right\}
$$

Mas como $v\left(T^{-1}(C)=v(C)=\mu(C)\right.$ para todo $C \in \mathscr{A}$, temos que $\mu\left(T^{-1}(B)=\mu(B)\right.$ para todo $B \in \mathscr{B}, \operatorname{logo},(X, \mathscr{B}, \mu, T)$ é um sistema dinâmico preservativo.

Finalmente, seja $A^{\prime}=D_{0}$ e note que $\varphi\left(A^{\prime}\right)=A, T^{-n}\left(A^{\prime}\right)=D_{n}$ para todo $n \in \mathbb{N}$ e $\varphi\left(D_{n}\right)=$ $(-n+A) \cap \mathbb{N}, \log 0$

$$
\mu\left(A^{\prime}\right)=v\left(A^{\prime}\right)=p-\lim _{n \rightarrow \infty} \frac{\left|\varphi\left(A^{\prime}\right) \cap I_{n}\right|}{\left|I_{n}\right|}=p-\lim _{n \rightarrow \infty} \frac{\left|A \cap I_{n}\right|}{\left|I_{n}\right|}=\alpha .
$$

Agora seja $F \subset \mathbb{N}$ finito:

$$
\begin{aligned}
\mu\left(A^{\prime} \cap \bigcap_{n \in F} T^{-n}\left(A^{\prime}\right)\right) & =\mu\left(D_{0} \cap \bigcap_{n \in F} D_{n}\right) \\
& =v\left(D_{0} \cap \bigcap_{n \in F} D_{n}\right) \\
& =p-\lim _{n \rightarrow \infty} \frac{\left|\left(\varphi\left(D_{0}\right) \cap \bigcap_{n \in F} \varphi\left(D_{n}\right)\right) \cap I_{n}\right|}{\left|I_{n}\right|} \\
& =p-\lim _{n \rightarrow \infty} \frac{\left|\left(A \cap \bigcap_{n \in F}(-n+A)\right) \cap I_{n}\right|}{\left|I_{n}\right|} \\
& \leq d^{*}\left(\bigcap_{n \in F}(A \cap(-n+A))\right.
\end{aligned}
$$

Uma das formulações do Teorema da Recorrência de Poincaré diz que para qualquer sistema dinâmico $(X, \mathscr{B}, \mu, T)$, todo $k \in \mathbb{N}$ e todo $A \subset X$ com $\mu(A)>0$, existem números $n_{1}, \ldots, n_{k} \in \mathbb{N}$ de modo que $\mu\left(A \cap T^{n_{1}}(A) \cap \cdots \cap T^{n_{k}}(A)\right)>0$. Com esse resultado e o Princípio da Correspondência podemos concluir que dado qualquer $E \subset \mathbb{N}$ com $d^{*}(E)>0$ e qualquer $k \in \mathbb{N}$, existe $n_{1}, \ldots, n_{k}$ tais que $d^{*}\left(E \cap\left(E-n_{1}\right) \cap \cdots \cap\left(E-n_{k}\right)\right)>0$, em particular $E \cap(E-$ $\left.n_{1}\right) \cap \cdots \cap\left(E-n_{k}\right) \neq \emptyset$ o que por sua vez implica que existe $e \in E$ tal que $e+n_{1}, \ldots, e+n_{k} \in E$. Para provar que $E$ contém progressões aritméticas arbitrariamente longas, basta conseguir uma versão mais forte do Teorema de Recorrência de Poincaré.

Teorema 6.9 (Furstenberg). Seja $(X, \mathscr{B}, \mu)$ um espaço de probabilidade e funções que preservam medida $T_{1}, T_{2}, \ldots, T_{l}: X \rightarrow X$ que comutam entre si. Seja $B \in \mathscr{B}$ com $\mu(B)>0$, então existe $d \in \mathbb{N}$ tal que $\mu\left(B \cap \bigcap_{i=1}^{l} T_{i}^{-d}(B)\right)>0$. 
Nos resultados até aqui expostos da Teoria de Ramsey concluímos que para qualquer partição finita existe um conjunto da partição possuindo a propriedade de interesse (ser um IP-set, por exemplo), mas não conseguimos determinar qual deles. O Teorema de Szemerédi nos dá uma condição necessária para um conjunto ser AP-rich, esta condição é ser um conjunto "grande" (com densidade maior que 0 ).

Corolário 6.10 (Szemerédi). Seja $A \subset \mathbb{N} \operatorname{com} d^{*}(A)>0$, então $A$ é AP-rich.

Demonstração. Seja $(X, \mathscr{B}, \mu, T)$ o sistema dinâmico preservativo e $A^{\prime} \in \mathscr{B}$ dados pelo Teorema 6.8.

Seja $l \in \mathbb{N}$ e para cada $i \in\{1, \ldots, l\}$ defina $T_{i}=T^{i}$, então $T_{1}, \ldots, T_{l}$ são ações preservativas que comutam entre si, então pelo lema anterior existe $d \in \mathbb{N}$ de modo que $\mu\left(A^{\prime} \cap \bigcap_{i=1}^{l} T_{i}^{-d}\left(A^{\prime}\right)\right)>0$ e note que $T_{i}^{-d}=T^{-i d}$. Defina $F=\{d, 2 d, \ldots, l d\}$. Então, novamente pelo Teorema 6.8,

$$
d^{*}\left(A \cap \bigcap_{n \in F}(-i+A)\right) \geq \mu\left(A^{\prime} \cap \bigcap_{n \in F} T^{-n}\left(A^{\prime}\right)\right)>0 .
$$

Portanto existe $a \in A \cap \bigcap_{n \in F}(-i+A)$, o que implica $\{a, a+d, a+2 d, \ldots, a+l d\} \subset A$.

\subsection{Teorema de Schur}

O Teorema de Schur, mencionado no começo do Capítulo 5, pode ser formulado de maneira análoga ao que fizemos na demonstração do Corolário 6.10, utilizando a interseção entre um conjunto e suas translações.

Teorema 6.11. Seja $\mathbb{N}=\bigcup_{i=1}^{r} A_{i}$ uma partição finita de $\mathbb{N}$, então existe $j \in\{1, \ldots, r\}$ e $n \in A_{j}$ de modo que $A_{j} \cap\left(-n+A_{j}\right) \neq \emptyset$.

De fato, se existir $m \in A_{j} \cap-n+A_{j}$, então $m \in\left(-n+A_{j}\right)$ e portanto $m+n \in A_{j}$, ou seja, $\{m, n, m+n\} \subset A_{j}$. Por outro lado, se $\{m, n, m+n\} \subset A_{j}$, então $n+m \in A_{j}$ e, assim, $m \in\left(-n+A_{j}\right)$, em particular, $A \cap\left(-n+A_{j}\right) \neq \emptyset$.

Assim formulado é fácil ver que este teorema é um corolário do Lema 5.17, pois se $p$ é um ultrafiltro idempotente não principal e $A \in p$, então existe $n \in A^{*}(p)$ com $-n+A^{*}(p) \in p$ e $A^{*}(p) \subset A, \log 0 A \cap-n+A \neq \emptyset$. Em particular, temos que se $p$ é um ultrafiltro idempotente não principal e $A \in p$, então existem $m, n \in \mathbb{N} \operatorname{com}\{m, n, m+n\} \in A$.

Note que o resultado de densidade análogo ao Teorema de Szemerédi é claramente falso, já que $2 \mathbb{N}+1$ é um conjunto de densidade não nula, mas não contém uma tripla $\{m, n, m+n\}$, pois a soma de quaisquer dois números ímpares é um número par.

Nosso intuito, então, é mostrar que, em alguma das partes de uma partição finita, os conjuntos da forma $\{m, n, m+n\}$ são abundantes, no sentido de os conjuntos de possibilidades para $m$ e $n$ terem densidade não nula.

A demonstração do Teorema a seguir é baseada em Gillis (1936). 
Lema 6.12. Seja $(X, \mathscr{B}, \mu, T)$ um sistema dinâmico e sejam $a, \varepsilon>0$. Se para cada $n \in \mathbb{N}$ temos $B_{n} \in \mathscr{B}$ com $\mu\left(B_{n}\right) \geq a$, então existem $i, j \in \mathbb{N}$ de modo que $\mu\left(B_{i} \cap B_{j}\right)>a^{2}-\varepsilon$.

Demonstração. Vamos mostrar que se para cada $k \in \mathbb{N}$ temos um conjunto $A_{k} \in \mathscr{B} \operatorname{com} \mu\left(A_{k}\right) \geq$ $a>0$, então existem $i, j \in \mathbb{N}$ de modo que $\mu\left(A_{i} \cap A_{j}\right) \geq a^{2}-\varepsilon$. Dado $A \subset X$, denotamos por $\chi_{A}$ a função característica de $A$, então é claro que $\mu(A)=\int \chi_{A}$ e $\chi_{A}^{2}=\chi_{A}$. Portanto para todo $k \in \mathbb{N}$, temos:

$$
\begin{aligned}
\left(\sum_{i=1}^{k} \mu\left(A_{i}\right)\right)^{2} & =\left(\int \sum_{i=1}^{k} \chi_{A_{i}}\right)^{2} \leq \int\left(\sum_{i=1}^{k} \chi_{A_{i}}\right)^{2}=\int \sum_{i=1}^{k} \chi_{A_{i}}+2 \sum_{i<j \leq k} \chi_{A_{i}} \chi_{A_{j}} \\
& =\sum_{i=1}^{k} \mu\left(A_{i}\right)+2 \sum_{i<j \leq k} \mu\left(A_{i} \cap A_{j}\right)
\end{aligned}
$$

A primeira desigualdade é uma consequência da desigualdade de Cauchy-Schwarz para integrais e do fato que $\int 1_{X}=\mu(X)=1$.

$$
\begin{aligned}
\sum_{i<j \leq k} \mu\left(A_{i} \cap A_{j}\right) \geq & \frac{1}{2}\left[-\sum_{i=1}^{k} \mu\left(A_{i}\right)+\left(\sum_{i=1}^{k} \mu\left(A_{i}\right)\right)^{2}\right]=\frac{1}{2}\left(\sum_{i=1}^{k} \mu\left(A_{i}\right)\right)\left(-1+\sum_{i=1}^{k} \mu\left(A_{i}\right)\right) \\
& \geq \frac{1}{2} k a(k a-1)
\end{aligned}
$$

Note que temos $\frac{1}{2} k(k-1)$ valores participando da soma, então para algum par $i, j \leq k$, temos

$$
\mu\left(A_{i} \cap A_{j}\right) \geq \frac{\frac{1}{2} k a(k a-1)}{\frac{1}{2} k(k-1)}=\frac{a(k a-1)}{k-1}=\frac{a^{2}\left(k-\frac{1}{a}\right)}{k-1} .
$$

$\mathrm{E} \lim _{k \rightarrow \infty} \frac{a^{2}\left(k-\frac{1}{a}\right)}{k-1}=a^{2}$, portanto para $k$ suficientemente grande, temos $\mu\left(A_{i} \cap A_{j}\right) \geq a^{2}-\varepsilon$

Para todo $N \subset \mathbb{N}$, denotaremos por $\mathscr{D}(N)$ o conjunto $\{m-n \in \mathbb{N} \mid m>n$ e $m, n \in N\}$.

Corolário 6.13. Seja $(X, \mathscr{B}, \mu, T)$ um sistema dinâmico que preserva medida. Para todo $N \subset \mathbb{N}$ infinto, todo $B \in \mathscr{B} \operatorname{com} \mu(B)>0$ e $\varepsilon>0$, existe $n \in \mathscr{D}(N)$ tal que $\mu\left(B \cap T^{n}(B)\right)>\mu(B)^{2}-\varepsilon$.

Demonstração. Enumere o conjunto $N=\left\{n_{k} \mid k \in \mathbb{N}\right\}$ e para cada $k \in \mathbb{N}$, defina $B_{k}=T^{n_{k}}(B)$, então $\mu\left(B_{k}\right)=\mu(B)$. Pelo lema anterior, existem $i, j \in \mathbb{N}$ de modo que

$$
\mu\left(B_{i} \cap B_{j}\right)=\mu\left(T^{n_{i}}(B) \cap T^{n_{j}}(B)\right)>\mu(B)^{2}-\varepsilon .
$$

Então para $n_{i}>n_{j}$,

$$
\mu\left(T^{n_{i}}(B) \cap T^{n_{j}}(B)\right)=\mu\left(T^{-n_{j}}\left(T^{n_{i}}(B) \cap T^{n_{j}}(B)\right)\right)=\mu\left(T^{n_{i}-n_{j}}(B) \cap B\right) .
$$


Os dois próximos resultados servirão para garantir que se particionarmos finitamente qualquer conjunto de densidade total, então existe um conjunto $N \subset \mathbb{N}$ infinito de modo que $\mathscr{D}(N)$ esteja contido em um mesmo elemento da partição. O corolário anterior garantirá que para o conjunto $B$ de medida não nula dado pelo Princípio da Correspondência de Furstenberg, existe um $n \in \mathscr{D}(N)$ de modo a medida da interseção $B \cap T^{n}(B)$ é não nula.

Lema 6.14. Seja $A \subset \mathbb{N}$. Se $d^{*}(A)=1$, então existe um conjunto $N \subset \mathbb{N}$ infinito tal que $\mathscr{D}(N) \subset$ A.

Demonstração. Se $d^{*}(A)=1$, então para todo $k \in \mathbb{N}$, existe $a \in A$ de modo que $\{a, a+1, a+$ $2, \ldots, a+k\} \subset A$ (pela definição de $d^{*}$ ). Defina $n_{1}=\min (A)$ e vamos construir o conjunto $N$ indutivamente.

Considere $a_{1} \in A$ de modo que $\left[a_{1}, a_{1}+n_{1}\right] \subset A$ e defina $n_{2}=a_{1}+n_{1}$, então $n_{2}-n_{1}=\left(a_{1}+\right.$ $\left.n_{1}\right)-n_{1}=a_{1} \in A$.

Do mesmo modo, tome $a_{2} \in A$ de modo que $\left[a_{2}, a_{2}+n_{2}\right] \subset A$ e defina $n_{3}=a_{2}+n_{2}$, então novamente $n_{3}-n_{2}=a_{2} \in A$ e $n_{3}-n_{1}=a_{2}+a_{1}$. Como $a_{1}<n_{2}$, temos que $n_{3}-n_{1}=a_{2}+a_{2} \in$ $\left[a_{2}, a_{2}+n_{2}\right] \subset A$.

Dados $n_{1}, \ldots, n_{k}$ de modo que $n_{i}>n_{i+1}$ para todo $1 \leq i \leq k$ e $\left\{n_{i}-n_{j} \mid i>j\right\} \subset A$, existe $a_{k} \in A$ de modo que $\left[a_{k}, a_{k}+n_{k}\right] \subset A$ e defina $n_{k+1}=a_{k}+n_{k}$, e novamente $n_{k+1}-n_{j} \in A$ para todo $1 \leq j \leq k$.

Defina portanto $N=\left\{n_{k} \mid k \in \mathbb{N}\right\}$, e temos que $n_{i}-n_{j} \in A$ para todo $i>j$.

Lema 6.15. Seja $A \subset \mathbb{N}$ de modo que exista $N \subset \mathbb{N}$ infinito com $\mathscr{D}(N) \subset A$. Se $A=\bigcup_{i=1}^{r} A_{i}$ é uma partição finita de $A$, então existem $j \in\{1, \ldots, r\}$ e $M \subset \mathbb{N}$ infinito com $\mathscr{D}(M) \subset A_{j}$.

Demonstração. Considere a enumeração $N=\left\{n_{1}, n_{2}, \ldots\right\}$. Defina a partição $[\mathbb{N}]^{2}=\bigcup_{i=1}^{r} B_{i}$ como $\{a, b\} \in B_{i}$ se, e somente se, $n_{a}-n_{b} \in A_{i}$ ou $n_{b}-n_{a} \in A_{i}$. Pelo Teorema de Ramsey (5.7), existem $j \in\{1, \ldots, r\}$ e um conjunto $R \subset \mathbb{N}$ infinito tal que $[R]^{2} \subset B_{j}$. Considere $M=\left\{n_{r} \mid r \in R\right\}$, então $M \subset N$ e pela definição de $B_{j}$, temos que $\mathscr{D}(M) \subset A_{j}$.

Chegamos finalmente ao teorema principal. Para $A \subset \mathbb{N}$, vimos que se existir $n \in A$ de modo que $A \cap(-n+A) \neq \emptyset$ então para todo $m \in A \cap(-n+A)$ temos que $\{m, n, m+n\} \subset A$. O Teorema de Schur, portanto, nos diz que existe $n \in \mathbb{N} \operatorname{com} A \cap(-n+A) \neq \emptyset$.

A generalização mais imediata deste resultado seria mostrar que existe $n \in \mathbb{N}$ de modo que $d^{*}(A \cap(-n+A))>0$, o que significa que o conjunto dos $m \in \mathbb{N}$ de modo que $\{m, n, m+n\} \in A$ tem densidade não nula. O que vamos mostrar é que não existe apenas um $n \in \mathbb{N}$ satisfazendo $d^{*}(A \cap(-n+A))>0$, e sim um conjunto de densidade não nula.

Teorema 6.16. Seja $\mathbb{N}=\bigcup_{i=1}^{r} A_{i}$ uma partição finita de $\mathbb{N}$. Existe $j \in\{1, \ldots, r\}$ de modo que $d^{*}\left(A_{j}\right)>0$ e para todo $\varepsilon>0$,

$$
\left\{n \in A_{j} \mid d^{*}\left(A_{j} \cap\left(-n+A_{j}\right)\right) \geq d^{*}\left(A_{j}\right)^{2}-\varepsilon\right\}
$$


é um conjunto de densidade não nula.

Demonstração. Sem perda de generalidade, vamos supor que existe $k \in\{1, \ldots, r\}$ tal que $d^{*}\left(A_{i}\right)>0$ para $1 \leq i \leq k$ e $d^{*}\left(A_{i}\right)=0$ para $k<i \leq r$, então é claro que $d^{*}\left(\bigcup_{i=1}^{k} A_{i}\right)=1$. Seja $\varepsilon>0$ dado e para cada $i \in\{1, \ldots, k\}$, defina o conjunto

$$
R_{i}=\left\{n \in A_{i} \mid d^{*}\left(A_{i} \cap\left(-n+A_{i}\right)\right) \geq d^{*}\left(A_{i}\right)^{2}-\varepsilon\right\} .
$$

Para que a demonstração esteja completa basta mostrar que algum dos $R_{i}$ tem densidade não nula.

Suponha $d^{*}\left(R_{i}\right)=0$ para todo $i \in\{1, \ldots, k\}$. Defina $C_{i}=A_{i} \backslash R_{i}$ para cada $i \in\{1, \ldots, k\}$, então $d^{*}\left(C_{i}\right)=d^{*}\left(A_{i}\right)$ e, assim, para cada $n \in C_{i}, d^{*}\left(C_{i} \cap\left(-n+C_{i}\right)\right)<d^{*}\left(C_{i}\right)^{2}-\varepsilon$. Note que $d^{*}\left(\bigcup_{i=1}^{k} C_{i}\right)=1$, portanto pelo Lema 6.14, existe $N \subset \mathbb{N}$ infinito com $\mathscr{D}(N) \subset \bigcup_{i=1}^{k} C_{i}$. Pelo Lema 6.15 existe $j \in\{1, \ldots, k\}$ e $M \subset \mathbb{N}$ infinito com $\mathscr{D}(M) \subset C_{j}$, já o Corolário 6.13 garante que para todo sistema dinâmico $(X, \mathscr{B}, \mu, T)$, todo $B \in \mathscr{B} \operatorname{com} \mu(B)>0$ e $\varepsilon>0$, existe $n \in C_{j}$ tal que $\mu\left(B \cap T^{n}(B)\right)>\mu(B)^{2}-\varepsilon$. Em particular, podemos tomar $(X, \mathscr{B}, \mu, T)$ e $B \in \mathscr{B}$ com $\mu(B)=d^{*}\left(C_{j}\right)$ como garantido pelo Princípio da Correspondência de Furstenberg, então

$$
d^{*}\left(C_{j} \cap\left(-n+C_{j}\right)\right) \geq \mu\left(B \cap T^{n}(B)\right) \geq \mu(B)^{2}-\varepsilon=d^{*}\left(C_{j}\right)^{2}-\varepsilon .
$$

Contradizendo a definição de $C_{j}$. Logo existe $j \in\{1, \ldots, k\}$ tal que $d^{*}\left(R_{j}\right)>0$.

\subsection{Teorema de Brauer}

No Teorema de Van der Waerden (Teorema 5.8), para cada $k \in \mathbb{N}$ conseguimos números $a, d \in \mathbb{N}$ de modo que o conjunto $\{a, a+d, a+2 d \ldots, a+k d\}$ esteja contido em uma das partes, mas não sabemos se a razão $d$ também estava nesta parte. Por outro lado, o Teorema de Schur 5.1 nos dá dois números distintos $a, d \in \mathbb{N}$ de modo que $\{d, a, a+d\}$ esteja na mesma parte. $\mathrm{O}$ Teorema de Brauer, portanto, funde estes dois conjuntos, mais precisamente:

Teorema 6.17 (Brauer). Seja $r \in \mathbb{N} e \mathbb{N}=\bigcup_{i=1}^{r} A_{i}$. Existe $i \leq r$ tal que para todo $k \in \mathbb{N}$, existem $a, d \in \mathbb{N}$ de modo que

$$
\{d, a, a+d, a+2 d, \ldots, a+k d\} \subset A_{i} .
$$

Outra forma de enunciar o teorema é: para toda partição finita $\mathbb{N}=\bigcup_{i=1}^{r} A_{i}$, existe $j \in\{1, \ldots, r\}$ de modo que para todo $k \in \mathbb{N}$, existe $d \in A_{j}$ com

$$
A_{j} \cap \bigcap_{i=1}^{k}\left(-i d+A_{j}\right) \neq \emptyset
$$

ou, alternativamente, para todo $k \in \mathbb{N}$

$$
\left\{d \in A_{j} \mid A_{j} \cap \bigcap_{i=1}^{k}\left(-i d+A_{j}\right) \neq \emptyset\right\} \neq \emptyset .
$$


Assim, $a \in A_{j} \cap \bigcap_{i=1}^{k}\left(-i d+A_{j}\right)$ significa que para cada $i \in\{1, \ldots, k\}, a \in(-i d+A)$ e, logo, $a+i d \in A$.

Novamente, como já mencionado sobre o Teorema de Schur, nem todo conjunto de densidade não nula tem essa propriedade, então o que vamos mostrar é que podemos fortalecer o resultado garantindo na última formulação do Teorema de Brauer que tanto a interseção, quanto o conjunto todo, tenham densidade não nula.

Lema 6.18. Para todo sistema dinâmico $(X, \mathscr{B}, \mu, T)$ e quaisquer $A \in \mathscr{B} \operatorname{com} \mu(A)>0, k \in \mathbb{N}$ e $C \subset \mathbb{N}$ infinito, existe $d \in F S(C)$ tal que

$$
\mu\left(A \cap T^{-d}(A) \cap T^{-2 d}(A) \cap \cdots \cap T^{-k d}(A)\right)>0 .
$$

Demonstração. Furstenberg e Katznelson (1985) Teorema A.

O Teorema 6.9 nos dá exatamente a mesma conclusão, porém não nos permite restringir o elemento $d$ como aqui. Novamente usamos os três ingredientes de nossas demonstrações de densidade: um ultrafiltro $p \in \Delta^{*}$ (neste caso, idempotente); o Princípio da Correspondência de Furstenberg; e o resultado de recorrência em sistemas dinâmicos com medida correspondente.

Teorema 6.19. Seja $p \in \Delta^{*}$ um ultrafiltro idempotente. Para todo $A \in p$ e $k \in \mathbb{N}$, temos

$$
\left\{d \in A \mid d^{*}\left(A \cap \bigcap_{i=1}^{k}(-i d+A)\right)>0\right\} \in p .
$$

Demonstração. Seja $D=\left\{d \in A \mid d^{*}\left(A \cap \bigcap_{i=1}^{k}(-i d+A)\right)>0\right\}$ e suponha que $D \notin p$. Como $p$ é ultrafiltro, $A \backslash D \in p$, e portanto existe $C \subset A \backslash D$ infinito tal que $F S(C) \subset A \backslash D$.

Pelo Princípio da Correspondência (Teorema 6.8), existe um sistema preservativo $(X, \mathscr{B}, \mu, T)$ e algum $B \in \mathscr{B}$ tal que $\mu(B)=d^{*}(A)>0$ e para todo $F \subset \mathbb{N}$ finito, $d^{*}\left(A \cap \bigcap_{n \in F}-n+A\right) \geq$ $\mu\left(B \cap \bigcap_{n \in F} T^{-n}(B)\right)$. Pelo lema anterior, existe $d \in F S(C)$ tal que

$$
\mu\left(B \cap T^{-d}(B) \cap \cdots \cap T^{-k d}(B)\right)>0 \Rightarrow d^{*}(A \cap(-d+A) \cap \cdots \cap(-k d+A))>0 .
$$

Mas então pela definição de $D$, temos que $d \in D$, o que é uma contradição, pois tomamos $d \in F S(C) \subset A \backslash D$.

Corolário 6.20. Seja $r \in \mathbb{N}$ e $\mathbb{N}=\bigcup_{i=1}^{r}$. Existe $j \in\{1, \ldots, r\}$ tal que para todo $k \in \mathbb{N}, d^{*}(\{d \in$ $\left.\left.A_{i} \mid A \cap(-d+A) \cap \cdots \cap(-k d+A)\right)>0\right\}>0$.

\subsection{Teorema de Hindman e densidade}

Assim como no Teorema de Schur, os números ímpares são um exemplo de conjunto de densidade não nula que não é um IP-set, mas ainda assim existe uma relação forte entre os IP-sets e densidade. 
Dada uma partição finita qualquer, sabemos que uma das partes é um IP-set e que uma das partes (não necessariamente a mesma) tem densidade não nula. Não só vamos mostrar que podemos exigir que uma das partes seja um IP-set de densidade não nula, mas que, de alguma forma, esse conjunto contém muitos IP-sets.

Diferente dos resultados anteriores, aqui faremos a demonstração combinatória, utilizando ultrafiltros como ferramenta secundária.

Lema 6.21. Seja $A \subset \mathbb{N}$, então $d^{*}(A)=d^{*}(-t+A)$ para todo $t \in \mathbb{N}$.

Demonstração. Em primeiro lugar, note que para todo $A \subset \mathbb{N}$ e $t, n \in \mathbb{N}$ temos

$$
|A \cap[1, n]|-t \leq|(-t+A) \cap[1, n]| \leq|A \cap[1, n]|+t .
$$

Portanto

$$
\begin{gathered}
d^{*}(A)=\lim _{n \rightarrow \infty} \sup \frac{|A \cap[1, n]|}{n} \leq \lim _{n \rightarrow \infty} \sup \frac{|(-t+A) \cap[1, n]|+t}{n}=d^{*}(-n+A) \mathrm{e} \\
d^{*}(-t+A)=\lim _{n \rightarrow \infty} \sup \frac{|(-t+A) \cap[1, n]|}{n} \leq \lim _{n \rightarrow \infty} \sup \frac{|A \cap[1, n]+t|}{n}=d^{*}(A) .
\end{gathered}
$$

Teorema 6.22. $O$ conjunto $\Delta^{*} \subset \beta \mathbb{N}$ é um ideal à esquerda fechado de $(\beta \mathbb{N},+)$.

Demonstração. Para mostrar que $\Delta^{*}$ é fechado, tome $p \notin \Delta^{*}$, então por definição existe $A \in p$ com $d^{*}(A)=0$, mas então $\bar{A}$ é uma vizinhança de $p$ e $\bar{A} \cap \Delta^{*}=\emptyset$.

Resta mostrar que $\Delta^{*}$ é um ideal, para isso temos que mostrar que dado $p \in \Delta^{*}, \beta S+p \subset \Delta^{*}$. Pela Proposição 3.16, temos que $\beta \mathbb{N}+p=\overline{\mathbb{N}}+p$, e como $\Delta^{*}$ é fechado, basta mostrarmos que $\mathbb{N}+p \subset \Delta^{*}$.

Seja $p \in \Delta^{*}$ e $t \in \mathbb{N}$, então $t+p=\{A \subset \mathbb{N} \mid-t+A \in p\}$. Se $-t+A \in p$, então $d^{*}(-t+A)>0$, portanto $d^{*}(A)>0, \log t+p \in \Delta^{*}$.

Note que todo conjunto finito tem densidade nula, portanto se $p \in \Delta^{*}$, então todos os elementos de $p$ são infinitos, logo $p$ não é ultrafiltro principal. Além disso, pelo Corolário 3.18, temos que existe algum ultrafiltro idempotente não principal que pertence a $\Delta^{*}$. A primeira parte da nossa afirmação está, portanto, demonstrada, pois mostramos que existe $p \in \Delta^{*} \cap E(\beta \mathbb{N})$, ou seja, para todo $A \in p, A$ é um IP-set com $d^{*}(A)>0$.

Lema 6.23. Seja $A \subset \mathbb{N} \operatorname{com} d^{*}(A)>0$ e $B \subset \mathbb{N}$ infinito. Então para todo $\varepsilon>0$, existem $x, y \in B$ $\operatorname{com} x<y$ tais que

$$
d^{*}(A \cap(-(y-x)+A)) \geq d^{*}(A)^{2}-\varepsilon .
$$


Demonstração. Sejam $A, B \subset \mathbb{N}$ como no enunciado e fixe $\varepsilon>0$. Sejam $(X, \mathscr{B}, \mu, T)$ e $A^{\prime} \in \mathscr{B}$ com $\mu\left(A^{\prime}\right)=d^{*}(A)$ como no enunciado do Princípio da Correspondência de Furstenberg (6.8). Pelo corolário 6.13, existe $n \in \mathscr{D}(B)$ com

$$
\mu\left(A^{\prime}\right) \cap T^{n}\left(A^{\prime}\right)>\mu\left(A^{\prime}\right)^{2}-\varepsilon .
$$

Novamente, pelo Princípio da Correspondência de Furstenberg, temos

$$
\left.d^{*}(A \cap-n+A) \geq \mu\left(A^{\prime} \cap T^{n}\left(A^{\prime}\right)\right)=\mu\left(T^{-n}\left(A^{\prime}\right) \cap A^{\prime}\right)\right)>\mu\left(A^{\prime}\right)^{2}-\varepsilon=d^{*}(A)-\varepsilon .
$$

Como $n \in \mathscr{D}(B)$, existem $x, y \in B \operatorname{com} x>y$ de modo que $n=x-y$.

Lema 6.24. Seja $p \in \Delta^{*}$ idempotente. Se $A \in p$ e $\varepsilon>0$, então

$$
\{x \in A \mid(-x+A) \in p\} \in p \quad \text { e } \quad\left\{x \in A \mid d^{*}(A \cap(-x+A)) \geq d^{*}(A)^{2}-\varepsilon\right\} \in p .
$$

Em particular, a interseção destes conjuntos é um elemento de $p$.

Demonstração. Se $p$ é idempotente, temos

$$
A \in p \Leftrightarrow A \in p+p \Leftrightarrow\{x \in \mathbb{N} \mid-x+A \in p\} \in p,
$$

mas $\{x \in A \mid(-x+A) \in p\}=A \cap\{x \in \mathbb{N} \mid-x+A \in p\}$ e ambos os conjuntos da interseção são elementos de $p$, portanto o primeiro conjunto do enunciado pertence a $p$.

Defina $B:=\left\{x \in A \mid d^{*}(A \cap(-x+A)) \geq d^{*}(A)^{2}-\varepsilon\right\}$. Precisamos mostrar que $B \in p$.

Suponha, por contradição, que $B \notin p$, então $\mathbb{N} \backslash B \in p$. Como $p$ é idempotente, $\mathbb{N} \backslash B$ é um IP-set, ou seja, existe uma sequência $\left(x_{n}\right)_{n \in \mathbb{N}}$ tal que $F S\left(\left\{x_{n} \mid n \in \mathbb{N}\right\}\right) \subset \mathbb{N} \backslash B$. Defina $C=$ $\left\{\sum_{i=1}^{n} x_{i} \mid n \in \mathbb{N}\right\}$. Como $C$ é infinito, existem, pelo lema anterior, $m, n \in C$ com $n<m$ de modo que $d^{*}\left(\sum_{i=1}^{m} x_{i}-\sum_{i=1}^{n} x_{i}\right) \geq d^{*}(A)^{2}, \operatorname{logo}$

$$
\begin{gathered}
\sum_{i=1}^{m} x_{i}-\sum_{i=1}^{n} x_{i} \in B, \text { mas } \\
\sum_{i=1}^{m} x_{i}-\sum_{i=1}^{n} x_{i}=\sum_{i=n}^{m} x_{i} \in F S\left(\left\{x_{n} \mid n \in \mathbb{N}\right\}\right) \subset \mathbb{N} \backslash B,
\end{gathered}
$$

contradição.

Dado $A \subset \mathbb{N}$, uma função $f: \mathbb{N} \rightarrow A$ pode ser vista como uma forma de se determinar um conjunto $B \subset A$, em que a cada passo $n \in \mathbb{N}$, decidimos que o elemento $f(n)$ pertence a $B$, ou seja, a função $f$ determina o conjunto $B=\operatorname{Im}(f)$. Claramente podemos ter duas funções $f, g: \mathbb{N} \rightarrow A$ determinando o mesmo conjunto $B$, ou seja, $\operatorname{Im}(f)=B=\operatorname{Im}(g)$, mas ainda assim a forma como o construímos é diferente.

Uma árvore, portanto, não apenas determina uma família de subconjuntos de $A$, mas também as formas válidas de construção desses subconjuntos. 
Definição 6.25. Seja $A \subset \mathbb{N}$. Uma árvore sobre $A$ é um conjunto $T$ de funções em que para toda $f \in T:$

1. $\operatorname{dom}(f)=\{1,2, \ldots, n\}, n \in \mathbb{N}, \mathrm{e} \operatorname{Im}(f) \subset A$.

2. Se $f \in T$ e $\operatorname{dom}(f)=n>1$, então $f\{\{1, \ldots, n-1\} \in T$.

Um ramo de $T$ é uma função $g: \mathbb{N} \rightarrow A$ de modo que $g \uparrow_{\{1, \ldots, n\}} \in T$ para todo $n \in \mathbb{N}$.

Para cada $f \in T$ com $\operatorname{dom}(f)=\{1, \ldots, n\}$, definimos o conjunto

$$
B_{f}(T)=\left\{x \in A \mid \exists g:\{1, \ldots, n, n+1\} \rightarrow A \operatorname{com} g\left\lceil_{\{1, \ldots, n\}}=f, g(n+1)=x \text { e } g \in T\right\} .\right.
$$

No teorema seguinte temos um IP-set $A \subset \mathbb{N}$ com densidade não nula e estamos interessados nos conjuntos $H$ de modo que $F S(H) \subset A$. Considere todas as funções $g: \mathbb{N} \rightarrow A$ com $\operatorname{Im}(g)$ infinito de modo que $F S(\{g(t) \mid t \in \mathbb{N}\}) \subset A$ e construa a árvore $T$ sobre $A$ consistindo nas restrições dessas funções a segmentos iniciais de $\mathbb{N}$.

É claro que essa árvore é não vazia, existe $H \subset A$ com $F S(H) \subset A$, então qualquer função com imagem $H$ produz um ramo de $T$, mas temos dois fatos que não são óbvios: o primeiro é que para toda $f \in T$, temos que $B_{f}(T)$ tem densidade não nula, ou seja, temos muitas formas de continuar a função $f$ (item (3)); o segundo é que existem ramos $g$ de $T$ de modo que a interseção de $A$ por um elemento de $F S(\{g(t) \mid t \in \mathbb{N}\})$ tem densidade tão próxima quanto a de $A$ quanto quisermos.

Teorema 6.26. Seja $p \in \Delta^{*}$ um ultrafiltro idempotente, $A \in p$ e $0<\delta<d^{*}(A)$. Existe uma árvore T sobre A tal que:

1. Para cada ramo g de $T, F S(\{g(t) \mid t \in \mathbb{N}\}) \subset A$.

2. Para cada ramo g de $T$ e cada $F \subset \mathbb{N}$ finito

$$
d^{*}\left(A \cap \bigcap \left\{-y+A \mid y \in F S(\{g(t) \mid t \in F\})>\delta^{2^{|F|}} .\right.\right.
$$

3. Para toda $f \in T, d^{*}\left(B_{f}(T)\right)>0$.

Demonstração. Sejam $g \in T \operatorname{com} \operatorname{dom}(g)=\{1,2, \ldots, n\}$ e $F \subset \operatorname{dom}(g)$. Definimos os conjuntos:

$$
\begin{gathered}
D(F, g)=A \cap \bigcap\{-y+A \mid y \in F S(\{g(t) \mid t \in F\}) ; \\
\left.C(F, g)=\{x \in D(F, g) \mid-x+D(F, g)\} \in p \text { e } d^{*}(D(F, g) \cap(-x+D(F, g)))>\delta^{2^{|F|+1}}\right\} ; \mathrm{e} \\
B(g)=\bigcap\{C(F, g) \mid F \subset \operatorname{dom}(g)\} .
\end{gathered}
$$

Se $g$ é a função vazia, definimos $D(F, g)=A$, o que servirá como a base para a indução a seguir. Definimos indutivamente os conjuntos $\left(T_{n}\right)_{n \in \omega}$ como $T_{0}=\{\emptyset\}$ e

$$
T_{n+1}=\left\{g \cup\{n+1, x\} \mid g \in T_{n}, x \in B(g) \text { e } g \cup\{n+1, x\} \text { é estritamente crescente }\right\} .
$$


Por fim defina $T=\bigcup_{n \in \omega} T_{n}$. É claro que $T$ é uma árvore sobre $A$.

Vamos mostrar por indução sobre $n$ que para toda $g \in T_{n}$ e todo $F \subset\{1,2, \ldots, n\}, C(F, g) \in p$ e $d^{*}(D(F, g))>\delta^{2^{|F|}}$.

No caso $n=0, T_{0}=\{\emptyset\}$, então $g=F=\emptyset$. Como $D(F, g)=A, d^{*}(D(F, g))>\delta=\delta^{2^{|F|}}$. Tome $\varepsilon=d^{*}(A)^{2}-\delta^{2}$, então, pelo lema anterior, temos que

$$
C(F, g)=\left\{x \in A \mid-x+A \in p \text { e } d^{*}(A \cap(-x+A))>d^{*}(A)^{2}-\varepsilon\right\} \in p .
$$

Seja $k \in \omega$ e suponha que o resultado seja válido para todo $g \in T_{k}$ e todo $F \subset\{1, \ldots, k\}$. Sejam $g \in T_{k+1}$ e $F \subset\{1, \ldots, k+1\}$. Temos dois casos: $k+1 \notin F$ e $k+1 \in F$.

Para o primeiro caso defina $h=g \uparrow_{\{1, \ldots, k\}}$, temos que $h \in T_{K}$ e, $\operatorname{logo}, d^{*}(D(F, g))=d^{*}(D(F, h))>$ $\delta^{2^{|F|}}$ e $C(F, g)=C(F, h) \in p$ pela hipótese de indução.

Se $k+1 \in F$ defina $h=g\left\lceil_{\{0, \ldots, k\}}, H=F \backslash\{k+1\}\right.$ e $x=g(k+1)$. Temos que $h \in T_{k}$ e $x \in B(h)$. Afirmamos que

$$
D(F, g)=D(H, h) \cap(-x+D(H, h)) .
$$

Vamos mostrar que $D(H, h) \cap(-x+D(H, h))=D(F, g)$. Seja $z \in D(H, h) \cap(-x+D(H, h))$, então $z \in D(H, h) \subset A$. Considere $y \in F S(\{g(t) \mid t \in F\})$ e tome $G \subset F$ de modo que $y=$ $\sum_{t \in G} g(t)$, temos que mostrar que $z \in-y+A$. Se $k+1 \notin G$, temos que $y \in F S(\{g(t) \mid t \in H\})$, e como $z \in D(H, h)$, temos o resultado. Suponha portanto que $k+1 \in G$. Se $G=\{k+1\}$, então $y=x$ e portanto $z \in-x+D(H, h) \subset-x+A=-y+A$. Por fim, se $K=G \backslash\{k+1\} \neq \emptyset$, então $K \subset H$. Fixe $v=\sum_{t \in H} g(t)=\sum_{t \in H} h(t)$, temos que $v \in F S(\{h(t) \mid t \in H\})$ portanto $x+z \in$ $D(H, h) \subset-v+A$, assim, $v+x+z=y+z \in A$, ou seja, $z \in-y+A$.

Para mostrar que $D(F, g) \subset D(H, h) \cap(-x+D(H, h))$, seja $z \in D(F, g)$. Note que $F S(\{g(t) \mid t \in$ $H\}) \subset F S(\{g(t) \mid t \in F\})$, portanto $z \in D(H, h)$. Além disso, como $g(k+1)=x \in F S(\{g(t) \mid t \in$ $F\})$, temos que $x+z \in A$. Seja $y \in F S(\{g(t) \mid t \in H\})$, temos $y+x \in F S(\{g(t) \mid t \in F\})$, assim, $z \in-(y+x)+A$ o que implica $x+z \in-y+A$, ou seja, $z \in-x+D(H, h)$.

Completamos então a demonstração de que $D(F, g)=D(H, h) \cap(-x+D(H, h))$.

Note que $x \in B(h) \subset C(H, h)$ e $D(H, h) \supset C(H, h) \in p$, portanto $D(F, g)=D(H, h) \cap(-x+$ $D(H, h)) \in p$.

Como $x \in C(H, h)$, temos por definição que

$$
d^{*}(D(F, g))=d^{*}(D(H, h) \cap(-x+D(H, h)))>\delta^{2^{|H|+1}}=\delta^{2^{|F|}} .
$$

Seja $\gamma=d^{*}(D(F, g))-\delta^{2^{|F|}}$ e fixe $\varepsilon=2 \gamma \delta^{||^{|F|}}+\gamma^{2}>0$. Pelo lema anterior, temos:

$E=\left\{z \in D(F, g) \mid-z+D(F, G) \in p\right.$, e $\left.d^{*}(D(F, g) \cap(-z+D(F, g)))>d^{*}(D(F, g))^{2}-\varepsilon\right\} \in p$.

$\operatorname{Mas} d^{*}(D(F, g))^{2}-\varepsilon=\delta^{2^{|F|+1}}$, portanto $C(F, g)=E \in p$.

A indução está, assim, completa.

Seja $f: \omega \rightarrow A$ um ramo de $T$. Vamos mostrar por indução em $|F|$ que para todo $F \subset \mathbb{N}$ finito $\operatorname{com} n=\min F$ e $h=f\left\{_{\{1, \ldots, n-1\}}, \sum_{t \in F} f(t) \in D(\{1, \ldots, n-1\}, h)\right.$. 
Se $|F|=1$, ou seja, $F=\{n\}$, considere $g=f \uparrow_{\{1, \ldots, n\}}$ e $h=f \uparrow_{\{1, \ldots, n-1\}}$, então $g=h \cup\{n, f(n)\}$ em que:

$$
f(n) \in B(h) \subset C(\{1, \ldots, n-1\}, h) \subset D(\{1, \ldots, n-1\}, h) .
$$

Suponha então o resultado válido para todo $H \subset \mathbb{N} \operatorname{com} 1<|H|<k$ e seja $F \subset \mathbb{N}$ com $|F|=k$. Considere $G=F \backslash\{n\}, m=\min G, g=f \uparrow_{\{1, \ldots, n\}}, h=f \uparrow_{\{1, \ldots, n-1\}}$ e $j=f \uparrow_{\{1, \ldots, m-1\}}$. Note que $|G|<\ell$, portanto

$$
\sum_{t \in G} f(t) \in D(\{1, \ldots, m-1\}, j) \subset D(\{1, \ldots, n\}, g) .
$$

Mas provamos anteriormente que

$$
D(\{1, \ldots, n\}, g)=D(\{1, \ldots, n-1\}, h) \cap(-f(n)+D(\{1, \ldots, n-1\}, h),
$$

assim, $\sum_{t \in G} f(t)+f(n)=\sum_{t \in F} f(t) \in D(\{1, \ldots, n-1\}, h)$, completando a indução. Em particular, provamos o item (1) do enunciado.

Para o item (2), se $f$ é um ramo de $T$ e $F \subset \mathbb{N}$ com $\max F=n$, tome $h=f \uparrow_{\{1, \ldots, n\}}$, então $A \cap \bigcap\{-y+A \mid y \in F S(\{f(t) \mid t \in F\})=D(F, h)$, mas já foi demonstrado anteriormente que $d^{*}(D(F, h))>\delta^{2^{|F|}}$.

Por fim note que se $g \in t$, então $B(g)$ é interseção finita de elementos de $p$, portanto $B(g) \in p$, além disso, $B_{g}=\{x \in B(g) \mid x>g(n-1)\}$, ou seja, $B_{g} \subset B(g)$ e $\left|B(g) \backslash B_{g}\right|$ é finito, portanto $B_{g} \in p$.

\subsection{Alguns problemas em aberto}

O Teorema de Szemerédi (Corolário 6.10) nos garante que todo conjunto de densidade não nula é AP-rich, mas seriam esses os únicos conjuntos AP-rich? Ou haveria alguma noção de grandeza mais fundamental para caracterizar esses conjuntos?

Em 2004, Ben Green e Terence Tao (Green e Tao (2008)) mostraram que o conjunto dos primos é AP-rich, mas o Teorema do Número Primo (Newman (1980)) nos garante que eles tem densidade nula. Portanto a busca por uma noção de grandeza que caracterize os conjuntos AP-rich continua, e a principal conjectura em aberto, formulada por Erdős e Turán, é a seguinte:

Questão 6.27. Se $A=\left\{a_{n} \mid n \in \mathbb{N}\right\}$ de modo que $a_{n}<a_{n+1}$ para todo $n \in \mathbb{N}$ e $\sum_{n \rightarrow \infty} \frac{1}{a_{n}}=\infty$, então $A$ é um conjunto AP-rich?

Caso verdadeira, ela abarcaria todos os conjuntos AP-rich conhecidos, pois é um resultado de Euler que a soma dos recíprocos dos primos diverge (demonstração de Erdős em Aigner e Ziegler (1998)) e como mostraremos agora, qualquer conjunto de densidade não nula também tem esta propriedade.

Proposição 6.28. Seja $A=\left\{a_{n} \mid n \in \mathbb{N}\right\} \subset \mathbb{N}$ com $\left.a_{n}<a_{n+1}\right\}$ para todo $n \in \mathbb{N}$. Se $d^{*}(A)>0$, então a série $\sum_{n \rightarrow \infty} \frac{1}{a_{n}}$ é divergente. 
Demonstração. Seja $d^{*}(A)=\delta>0$. Primeiro vamos definir uma sequência $\left(n_{k}\right)_{k \in \mathbb{N}}$ começando com $n_{1}=0$ e de modo que para todo $k \in \mathbb{N}$,

$$
n_{k+1} \geq \frac{4}{\delta} n_{k} \mathrm{e}\left|A \cap\left[1, n_{k+1}\right]\right| \geq \frac{\delta}{2} n_{k+1} .
$$

Logo, por definição, temos que para todo $k \in \mathbb{N}$ :

$$
\left|A \cap\left(n_{k}, n_{k+1}\right]\right|=\left|A \cap\left[1, n_{k+1}\right]\right|-\left|A \cap\left[1, \ldots, n_{k}\right]\right| \geq \frac{\delta}{2} n_{k+1}-n_{k} .
$$

Note que para todo $n \in\left(n_{k}+1, \ldots, n_{k+1}\right]$, temos $\frac{1}{n} \geq \frac{1}{n_{k+1}}$.

$$
\begin{aligned}
\sum_{n \rightarrow \infty} \frac{1}{a_{n}} & \geq \sum_{k=1}^{\infty}\left\|A \cap\left(n_{k}, n_{k+1}\right]\right\| \frac{1}{n_{k+1}} \geq \sum_{k=1}^{\infty}\left(\frac{\delta}{2} n_{k+1}-n_{k}\right) \frac{1}{n_{k+1}} \\
& \geq \sum_{k=1}^{\infty}\left(\frac{\delta}{2} n_{k+1}-\frac{\delta}{4} n_{k+1}\right) \frac{1}{n_{k+1}}=\sum_{k=1}^{\infty} \frac{\delta}{4}
\end{aligned}
$$

Portanto a série diverge.

Algumas equivalências desta conjectura em termos de ultrafiltros foram obtidas por Neil Hindman, em Hindman (1988).

Digno de nota é a Erdös sumset conjecture, publicada em 1980, que foi demonstrada recentemente em Moreira, Richter e Robertson (2019).

Dizemos que um conjunto $A \subset \mathbb{N}$ é um sumset se existem conjuntos $B, C \subset \mathbb{N}$ infintos de modo que $B+C=\{b+c \in \mathbb{N} \mid b \in B, c \in C\} \subset A$. O fato de que toda partição finita de $\mathbb{N}$ contém algum sumset é consequência imediata do Teorema de Hindman, mas a conjectura, que foi demonstrada verdadeira, é a seguinte:

Teorema 6.29. Seja $A \subset \mathbb{N}$. Se $d^{*}(A)>0$, então $A$ é um sumset.

No artigo podemos encontrar questões sobre o tema que permanecem em aberto. Talvez a mais imediata delas seja:

Questão 6.30. Seja $A \subset \mathbb{N}$ e $k>2$. É verdade que se $d^{*}(A)>0$ então existem $B_{1}, \ldots, B_{k} \subset \mathbb{N}$ infinitos com $B_{1}+\cdots+B_{k} \subset A$ ?

Do mesmo modo que Green e Tao demonstraram que o conjunto dos números primos é AP-rich, os autores perguntam se os números primos são também um sumset. Um resultado parcial para esta pergunta já havia sido dado em 1990 em Granville (1990), com uma resposta afirmativa para a questão desde que se assuma a primeira conjectura de Hardy-Littlewood.

Questão 6.31. Existem conjuntos infinitos $A, B \subset \mathbb{N}$ de modo que $a+b$ é um número primo para quaisquer $a \in A$ e $b \in B$ ? 



\section{REFERÊNCIAS}

AIGNER, M.; ZIEGLER, M. Proofs from THE BOOK. Berlin: Springer-Verlag Berlin Heidelberg, 1998. Citado na página 112.

AURICHI, L. Notas de aula - Aplicações de Teoria dos Conjuntos. 2018. Disponível em: $<$ https://sites.icmc.usp.br/aurichi/lib/exe/fetch.php?media=curso:conjuntos2018.pdf>. Citado na página 26.

. Notas de aula - Topologia. 2020. Disponível em: <https://sites.icmc.usp.br/aurichi/lib/ exe/fetch.php?media=curso:topologia2020.pdf $>$. Citado na página 19.

BERGELSON, V. A density statement generalizing schur's theorem. Journal of Combinatorial Theory, v. 43, p. 338-343, 1986. Citado na página 97.

Ergodic Ramsey Theory - an Update. 1996. Citado na página 97.

BERGELSON, V.; BLASS, A.; HINDMAN, N. Partition theorems for spaces of variable words. Proceedings of the London Mathematical Society, s3-68, n. 3, p. 449-476, 1994. Disponível em: <https://londmathsoc.onlinelibrary.wiley.com/doi/abs/10.1112/plms/s3-68.3.449>. Citado na página 80 .

BERGELSON, V.; ROTHSCHILD, B. A selection of open problems. Topology and its Applications, v. 156, p. 2674-2681, 2009. Citado na página 94.

BLASS, A. Ultrafilters: Where topological dynamics = algebra = combinatorics. 1993. Citado na página 51 .

CLARK, P. Convergence. 2016. Disponível em: <http://math.uga.edu/ pete/convergence.pdf>. Citado na página 52.

DAVENPORT, D.; HINDMAN, N.; LEADER, I. Continuous homomorphisms on $\beta \mathbb{N}$ and ramsey theory. New York Journal of Mathematics, v. 6, p. 73-86, 2000. Citado nas páginas 49 e 95.

DOUWEN, E. The Čech-stone compactification of a discrete groupoid. Topology and its Applications, v. 39, n. 1, p. 43-60, 1991. ISSN 0166-8641. Disponível em: <http://www.sciencedirect. com/science/article/pii/016686419190074V>. Citado na página 50.

FURSTENBERG, H. Ergodic behavior of diagonal measures and a theorem of szemerédi on arithmetic progressions. Journal d'Analyse Mathématique, v. 31, n. 1, p. 204-256, Dec 1977. ISSN 1565-8538. Disponível em: <https://doi.org/10.1007/BF02813304>. Citado na página 97.

Recurrence in Ergodic Theory and Combinatorial Number Theory. Princeton, New Jersey: Princeton University Press, 1981. Citado nas páginas 19 e 97.

FURSTENBERG, H.; KATZNELSON, Y. An ergodic szemerédi theorem for ip-systems and combinatorial theory. Journal D'analyse Mathématique, v. 45, p. 117-168, 1985. Citado na página 107. 
GILLIS, J. Note on a property of measurable sets. Journal of the London Mathematical Society, s1-11, n. 2, p. 139-141, 1936. Disponível em: <https://londmathsoc.onlinelibrary.wiley. com/doi/abs/10.1112/jlms/s1-11.2.139>. Citado na página 103.

GOWERS, W. A new proof of szemerédi's theorem. Geometric \& Functional Analysis GAFA, v. 11, p. 465-588, 2001. Citado na página 95.

GRANVILlE, A. A note on sums of primes. Canadian Mathematical Bulletin, v. 33, n. 4, p. 452-454, 1990. Citado na página 113.

GREEN, B.; TAO, T. The primes contain arbitrarily long arithmetic progressions. Annals of Mathematics, v. 167, p. 481-547, 2008. Citado na página 112.

HINDMAN, N. Finite sums from sequences within cells of a partition of $n$. Journal of Combinatorial Theory, v. 17, p. 1-11, 1974. Citado na página 20.

Some equivalents of the erdös sum of reciprocals conjecture. European Journal of Combinatorics, v. 9, p. 39-47, 1988. Citado na página 113.

. Algebra in the Stone-Čech Compactification and its Applications to Ramsey Theory. 2005. Citado na página 21.

HINDMAN, N.; BERGELSON, V. Density versions of two generalizations of schur's theorem. Journal of Combinatorial Theory, v. 48, p. 32-38, 1988. Citado na página 97.

Nonmetrizable topological dynamics and ramsey theory. Transactions of the American Mathematical Society, v. 320, p. 293-320, 1990. Citado na página 50.

HINDMAN, N.; LEADER, I.; STRAUSS, D. Open problems in partition regularity. Combinatorics, Probability and Computing, Cambridge University Press, v. 12, n. 5 6, p. 571-583, 2003. Citado na página 94.

HINDMAN, N.; STRAUSS, D. Algebra in the Stone-Čech Compactification: Theory and Applications. Berlin/Boston: De Gruyter, 2012. Citado nas páginas 19, 37, 50, 53, 75, 93 e 97.

HINDMAN, N.; STRAUSS, D.; ZAMBONI, L. Recurrence in the dynamical system $\left(\mathrm{X},\left\langle\mathrm{T}_{s}\right\rangle_{s \in S}\right)$ and ideals of $\beta$ s. Indagationes Matematicae, v. 29, p. 293-312, 2018. Citado na página 51.

JECH, T. The Axiom of Choice. Amsterdam, New York.: North-Holland Pub. Co., American Elsevier Pub. Co., 1973. Citado na página 26.

KOURIL, M. Computing the van der waerden number $w(3,4)=293$. Integers, v. 12, n. A46, 2012. Citado na página 95.

MOREIRA, J.; RICHTER, F.; ROBERTSON, D. A proof of a sumset conjecture of erdős. Annals of Mathematics, Annals of Mathematics, Princeton U, v. 189, n. 2, p. 605, 2019. ISSN 0003-486X. Disponível em: <http://dx.doi.org/10.4007/annals.2019.189.2.4>. Citado na página 113.

NEWMAN, D. J. Simple analytic proof of the prime number theorem. The American Mathematical Monthly, v. 87, p. 693-696, 1980. Citado na página 112.

RICHANDSON, L. Measure and Integration: A Concise Introduction to Real Analysis. New Jersey: John Wiley \& Sons, 2009. Citado na página 100. 
SLEZIAK, M. Subnets. 2009. Disponível em: <http://thales.doa.fmph.uniba.sk/sleziak/texty/ rozne/topo/subnets2.pdf>. Citado na página 52.

WALKER, R. The Stone-Čech Compactification. Berlin: Springer-Verlag Berlin Heidelberg, 1974. Citado na página 29. 


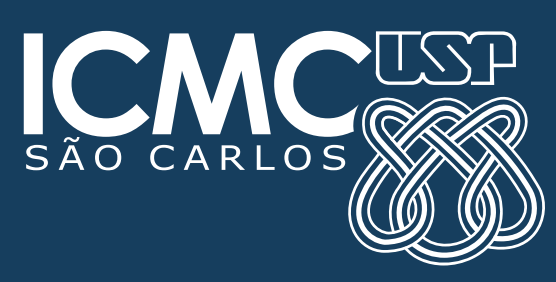

